\title{
Untersuchungen zum thermisch induzierten Luftwechselpotential von Kippfenstern
}

\begin{abstract}
Dissertation
zur Erlangung des Grades Doktor-Ingenieur

des Fachbereichs Architektur, Stadtplanung, Landschaftsplanung an der Universität Kassel
\end{abstract}

von

Monika Hall

Kassel 2004 


\author{
Als Dissertation vom \\ Fachbereich Architektur, Stadtplanung und Landschaftsplanung \\ angenommen am: 28.10.2003 \\ 1. Gutachter: Univ.-Prof. Dr.-Ing. Gerd Hauser, Universität Kassel \\ 2. Gutachter: Prof. Dr.rer.nat. Dr.-Ing.habil. Andreas Dillmann, DLR Göttingen
}

Tag der mündlichen Prüfung: 27.01.2004 
Hiermit versichere ich, dass ich die vorliegende Dissertation selbständig und ohne unerlaubte Hilfe und andere als die in der Dissertation angegebenen Hilfsmittel nicht benutzt habe. Alle Stellen, die wörtlich oder sinngemäß aus veröffentlichten oder unveröffentlichten Schriften entnommen sind, habe ich als solche kenntlich gemacht. Kein Teil dieser Arbeit ist in einem anderen Promotions- oder Habilitationsverfahren verwendet worden.

Kassel, den 
Die vorliegende Arbeit entstand neben meiner Tätigkeit als wissenschaftliche Mitarbeiterin am Fachgebiet Bauphysik im Fachbereich Architektur der Universität Kassel.

Mein besonderer Dank gilt Herrn Univ.-Prof. Dr.-Ing. Gerd Hauser, Fachgebiet Bauphysik, Universität Kassel, für die Anregung zu dieser Arbeit und die anschlieBende Förderung und Unterstützung.

Herrn Prof. Dr.rer.nat. Dr.-Ing. habil. Andreas Dillmann, Institut für Aerodynamik und Strömungstechnik, DLR Göttingen, danke ich für die Übernahme des Koreferats und für seine Betreuung.

Allen Kolleginnen und Kollegen am Fachgebiet Bauphysik, die zum Gelingen der Arbeit nicht unwesendlich beigetragen haben möchte ich meinen herzlichsten Dank aussprechen. Insbesondere sind hier Herr Dipl.-Ing. Markus Gehle und Herr Dipl.Ing. Martin Rösler hervorzuheben, die mir bei den messtechnischen Untersuchungen eine große Hilfe waren. Desweiteren möchte ich Herrn Dr.-Ing. Wolfram Haupt und Herrn Dr.-Ing. Achim Geißler danken, die mir mit Rat und Tat zur Seite standen. 


\section{Inhaltsverzeichnis}

Nomenklatur iv

1 Einleitung 1

2 Die natürliche Lüftung $\quad 3$

2.1 Allgemeines ....................... 3

2.2 Antriebskräfte ....................... 4

2.3 Bestimmung des Luftwechsels . . . . . . . . . . . . . 7

2.4 Fensterlüftung in der Literatur - ein Überblick . . . . . . . . . . . . 8

3 Thermisch induzierter Luftwechsel über Kippfenster 10

3.1 Allgemeines . . . . . . . . . . . . . . . . 10

3.2 Geometrie eines Kippfensters . . . . . . . . . . . . . . . 10

3.3 Ansatz Daler . . . . . . . . . . . . . . . . . . . . . . . . . . . 14

3.4 Ansatz Weberl . . . . . . . . . . . . . . . . . 14

3.5 Ansatz Weber $2 \ldots \ldots . \ldots . \ldots . . \ldots 15$

3.6 Ansatz Maas . . . . . . . . . . . . . . . . . . . . . . 15

3.7 Ansatz Zeidler . . . . . . . . . . . . . . . . . . . . . . . 16

3.8 Ansatz Asimakopoulos . . . . . . . . . . . . . . . . . . . . 17

3.9 Vergleich der Ansätze . . . . . . . . . . . . . . . . . . . . 17

4 Versuchsaufbau $\quad \mathbf{2 0}$

4.1 Modellraum und Messtechnik . . . . . . . . . . . . . . 20

4.2 Ablauf der Messungen . . . . . . . . . . . . . . . . . . 24

4.3 Messunsicherheit . . . . . . . . . . . . . . 25

5 Messergebnisse 27

5.1 Tracergas-Messungen . . . . . . . . . . . . . . 27

5.1.1 Darstellung der Zuluftvolumenströme . . . . . . . . . . 27

5.1.2 Vergleich unterschiedlicher Kippweiten . . . . . . . . 35

5.1.3 Einfluss von Heizung, Ventilatoren und Gardine . . . . . . . 36

5.1.4 Vergleich mit Ansätzen aus der Literatur . . . . . . . . . . . 39 
5.2 Temperaturverteilung . . . . . . . . . . . . . . . . . 41

5.2.1 Variante' ohne Laibung / ohne Heizung' . . . . . . . . . . . 41

5.2.2 Variante 'mit Laibung / ohne Heizung' . . . . . . . . . . 43

5.2.3 Variante 'mit Laibung / mit Heizung' . . . . . . . . . . . 43

5.2.4 Lufttemperaturen im fensternahen Bereich . . . . . . . . 45

5.2.5 Zusammenfassung der Temperaturverläufe . . . . . . . 47

6 Numerische Simulation $\quad 49$

6.1 Zur Simulation von Auftriebsströmungen . . . . . . . . . . . . 49

6.2 Grundlagen ........................ 50

6.3 Erstellung des Modells . . . . . . . . . . . . . . . 52

6.4 Modellparameter . . . . . . . . . . . . . . . . . 54

7 Vergleich Messung - CFD-Simulation $\quad 57$

7.1 Gegenüberstellung der Randbedingungen . . . . . . . . . . . . 57

7.2 Gegenüberstellung der Ergebnisse . . . . . . . . . . . . . . 57

7.2.1 Zuluftvolumenströme . . . . . . . . . . . 57

7.2.2 Temperaturen .................. 60

7.3 Stromlinien ....................... 64

8 Modellanpassung $\quad 69$

8.1 Modellansatz ... . . . . . . . . . . . . . . 69

8.2 Bestimmung der Öffnungsfläche . . . . . . . . . . . . . . . . 69

8.2.1 Allgemeines ................... 69

8.2.2 Fall 1: ohne Laibung . . . . . . . . . . . . . 73

8.2.3 Fall 2: Laibungstiefe kleiner als die Kippweite . . . . . . 73

8.2.4 Fall 3: Laibungstiefe größer als die Kippweite . . . . . . . . 74

8.3 Modellanpassung . . . . . . . . . . . . . . . . . 75

8.4 Ausblick . . . . . . . . . . . . . . . . . . 77

9 Schlussfolgerungen $\quad \mathbf{8 0}$

10 Zusammenfassung $\quad 82$ 


\section{Literatur}

12 Anhang $\quad 90$

12.1 Prozentuale Abweichung der einzelnen Varianten . . . . . . . . . . 90

12.2 Temperaturverläufe bei der Kippweite von $10 \mathrm{~cm}$. . . . . . . . . . 90

12.3 Temperaturverläufe bei der Kippweite von 6 cm . . . . . . . . . . 90

12.4 Temperaturverläufe bei der Kippweite von $3 \mathrm{~cm}$. . . . . . . . . . . 101

12.5 Korrelationskoeffizient . . . . . . . . . . . . . . 110

13 Bild- und Tabellenverzeichnis 111 


\section{Nomenklatur}

\begin{tabular}{|c|c|c|}
\hline a & $\mathrm{m}$ & variabler Abstand zwischen Kippflügel und Laibungssturz \\
\hline A & $\mathrm{m}^{2}$ & Fläche \\
\hline B & $\mathrm{m}$ & lichte Breite des Fensters \\
\hline $\mathrm{c}$ & ppm & Tracergas-Konzentration \\
\hline$c_{\mathrm{p}}$ & - & Winddruckbeiwert \\
\hline$c_{p}$ & $\mathrm{~J} /(\mathrm{kg} \mathrm{K})$ & spez. Wärmekapazität \\
\hline $\mathrm{C}_{\mathrm{d}}$ & - & Discharge-Koeffizient \\
\hline d & $\mathrm{m}$ & Rahmentiefe \\
\hline f & - & Reduzierfaktor \\
\hline $\mathrm{F}$ & $\mathrm{N}$ & Kraft \\
\hline $\mathrm{g}$ & $\mathrm{m} / \mathrm{s}^{2}$ & Erdbeschleunigung 9,81 \\
\hline $\mathrm{h}$ & $\mathrm{m}$ & reduzierte Höhe \\
\hline $\mathrm{H}$ & $\mathrm{m}$ & lichte Höhe des Fensters \\
\hline $\mathrm{m}$ & $\mathrm{m}$ & Abstand zwischen Rahmen und seitlicher Laibung \\
\hline $\mathrm{n}$ & - & Strömungsexponent \\
\hline $\mathrm{n}$ & $h^{-1}$ & Luftwechselrate \\
\hline $\mathrm{i}$ & - & Variable \\
\hline $\mathrm{j}$ & $\mathrm{m}$ & Abstand zwischen Blendrahmen und Laibungssturz \\
\hline $\mathrm{k}$ & - & Variable \\
\hline 1 & $\mathrm{~m}$ & Laibungstiefe \\
\hline $\mathrm{L}$ & $\mathrm{m}$ & charakteristische Länge \\
\hline $\mathrm{p}$ & $\mathrm{m}$ & Höhe des Bereichs, der nicht von der Laibung überdeckt wird \\
\hline $\mathrm{p}$ & $\mathrm{Pa}$ & Druck \\
\hline q & $\mathrm{m}$ & Höhe des Bereichs, der von der Laibung überdeckt wird \\
\hline $\mathrm{r}$ & - & Korrelationskoeffizient \\
\hline s & $\mathrm{m}$ & Kippweite \\
\hline $\mathrm{t}$ & $\mathrm{h}$ & Zeit \\
\hline $\mathrm{T}$ & $\mathrm{K}$ & Temperatur \\
\hline $\mathrm{u}$ & $\mathrm{m} / \mathrm{s}$ & Windgeschwindigkeit, Geschwindigkeit \\
\hline$V_{R}$ & $\mathrm{~m}^{3}$ & Raumvolumen \\
\hline$\dot{V}$ & $\mathrm{~m}^{3} / \mathrm{h}$ & Volumenstrom \\
\hline $\mathrm{w}$ & $\mathrm{m} / \mathrm{s}$ & charakteristische Geschwindigkeit \\
\hline $\mathrm{x}$ & $\mathrm{m}$ & Länge der Rahmenüberschneidung \\
\hline $\mathrm{y}$ & $\mathrm{m}$ & Abstand der beiden Rahmenteile, Abstand \\
\hline $\mathrm{Z}$ & $\mathrm{m}$ & Höhe \\
\hline $\mathrm{Z}$ & - & Faktor zur Bestimmung der neutralen Höhe \\
\hline
\end{tabular}


Nomenklatur (Fortsetzung)

$\begin{array}{lll}\alpha & - & \begin{array}{l}\text { Durchflusszahl } \\ \beta\end{array} \\ \beta & 1 / \mathrm{K} & \text { thermischer Ausdehnungskoeffizient } \\ \delta & & \text { Winkel } \\ \lambda & - & \text { Fehler einer Größe } \\ \lambda & \mathrm{W} /(\mathrm{m} \mathrm{K}) & \text { Wärmeleitfähigkeit } \\ \mu & \mathrm{Ns} / \mathrm{m}^{2} & \text { dynamische Viskosität } \\ \mu & - & \text { Einschnürungsfaktor } \\ \nu & \mathrm{m}^{2} / \mathrm{s} & \text { kinematische Viskosität } \\ \varphi & { }_{0} & \text { Winkel } \\ \rho & \mathrm{kg} / \mathrm{m}^{3} & \text { Dichte } \\ \tau & \mathrm{N} / \mathrm{m}^{2} & \text { Schubspannung } \\ \Theta & - & \text { Durchflussverhältnis }\end{array}$

\section{Indizes}

$\begin{array}{ll}\text { a } & \text { außen } \\ \text { A } & \text { Auftrieb } \\ \text { ber } & \text { berechnet } \\ \text { D } & \text { Daler } \\ \text { eff } & \text { effektiv } \\ \text { ges } & \text { gesamt } \\ \text { gem } & \text { gemessen } \\ \mathrm{i} & \text { innen, Laufindex } \\ \mathrm{n} & \text { neutral, Laufindex } \\ \mathrm{m} & \text { mittel } \\ \mathrm{max} & \text { Maximum } \\ \mathrm{min} & \text { Minimum } \\ \mathrm{M} & \text { Maas } \\ \mathrm{R} & \text { Reibung } \\ \mathrm{s} & \text { Kippweite } \\ \mathrm{t} & \text { turbulent } \\ \mathrm{T} & \text { Trägheit } \\ \mathrm{W} & \text { Wand } \\ \mathrm{W} 1 / \mathrm{W} 2 & \text { Weber1/Weber2 } \\ \mathrm{Z} & \text { Zeidler } \\ \text { zu } & \text { Zuluft }\end{array}$




\section{Einleitung}

Die sogenannte natürliche Lüftung - Lüftung infolge Temperatur- und Windeinwirkung über geöffnete Türen oder Fenster - ist auch heute noch in Wohngebäuden die am weitesten verbreitete Form des Lüftens. Selbst bei Gebäuden mit Lüftungsanlage entspricht die Fensteröffnungzeit in etwa der von Gebäuden mit Fensterlüftung [42]. Während des Lüftens wird verbrauchte Luft ab- und Frischluft einem Raum oder Gebäudebereich zugeführt. Der Luftwechsel kann über das Öffnen von Türen bzw. Fenstern oder über eine mechanisch betriebene Lüftungsanlage erfolgen und wird durch eine Luftwechselrate beschrieben. Sie ist definiert als das Verhältnis des innerhalb einer Stunde ein- bzw. ausgeströmten Volumenstroms zum belüfteten Raum- oder Gebäudevolumen.

Die Luftwechselrate ist im Hinblick auf energiesparendes Bauen für die Planung und Ausführung von Gebäuden eine wichtige Größe. Aus energetischen Gründen sollte diese möglichst gering gehalten werden. Ein ausreichender Luftwechsel ist jedoch für den hygienischen und bauphysikalischen Frischluftbedarf notwendig. Zur Abschätzung des Raumklimas sowie zur Vorbeugung von Bauschäden infolge Schimmelbildung ist daher die Kenntnis der Luftwechselrate ebenfalls wichtig.

Der Einsatz von Dreh-Kippfenstern sowie das Lüften über die Kippstellung ist in Deutschland üblich, so dass die Bestimmung des Luftwechsels über Kippfenster von großem Interesse ist. Es zeigt sich, dass die Anwendung von Ansätzen aus der Literatur zur Bestimmung des Luftwechsels zu unterschiedlichen Ergebnissen führt. Aus diesem Grund ist das Ziel dieser Arbeit die natürliche Lüftung über Kippfenster detaillierter zu quantifizieren und damit die Planungssicherheit für Kippfensterlüftung zu erhöhen. Die Arbeit gliedert sich in drei Teile:

- messtechnische Untersuchungen

- CFD-Simulationen

- Modellanpassung

Bei den messtechnischen Untersuchungen soll das Luftwechselpotential eines Kippfensters unter Berücksichtigung verschiedener Randbedingungen für die einseitige Fensterlüftung untersucht werden. Das Tracergas-Messverfahren wird zur Bestimmung der Volumenströme eingesetzt. Mit den Messdaten werden Informationen über den Einfluss von verschiedenen Kippwinkeln, Laibungstiefen und Heizungsanordnungen auf die Kipplüftung gewonnen. Die Messdaten sollen zum einen aus der Literatur bekannten Ansätzen für die Bestimmung des Zuluftvolumenstroms über Kippfenster gegenübergestellt und zum anderen mit Ergebnissen aus CFDSimulationen verglichen werden. Sie bilden im weiteren die Grundlage zu einer Modellanpassung.

Neben den Zuluftvolumenströmen werden auch die Lufttemperaturen an verschiedenen Stellen im Modellraum in Abhängigkeit von verschiedenen Randbedingungen messtechnisch bestimmt. Aus diesen Temperaturverläufen können Rückschlüsse 
über Lenkungseffekte einer innenliegenden Laibung und einer Heizung auf die Raumluftströmung abgeleitet werden.

Einige messtechnisch gewonnenen Ergebnisse - Volumenströme und Temperaturverteilungen - sollen im zweiten Teil der Arbeit mit CFD-Simulationen verglichen werden. Anwendungsmöglichkeiten und Grenzen der CFD-Simulationen werden dargestellt.

Im dritten Teil der Arbeit wird auf der Basis der messtechnischen Untersuchungen ein verbessertes Modell zur Beschreibung der einseitigen Kipplüftung entwickelt. Einerseits soll die Öffnungsfläche für den Zuluftvolumenstrom in Abhängigkeit von der Laibung bestimmt werden und andererseits ist das Vorhandensein einer Heizung während der Lüftungsdauer in dem Ansatz zu berücksichtigen.

Die Ergebnisse dieser Arbeit sollen dem Planer eine Hilfestellung sein, die natürliche, thermisch bedingte Lüftung über Kippfenster besser beschreiben zu können. Insbesondere die getrennte Betrachtung der o.g. einzelnen Einflüsse ermöglichen es, die Planung detaillierter durchzuführen. 


\section{Die natürliche Lüftung}

\subsection{Allgemeines}

Das Lüften von Gebäuden ist aus den verschiedensten Gründen unerlässlich. Durch das Lüften wird z.B. Feuchtigkeit aus dem Gebäude transportiert. Da Luftfeuchtigkeit u.a. ein entscheidender Faktor in Hinblick auf eine mögliche Schimmelpilzbildung ist [53], kann mit konsequentem Lüften diesem entgegen gewirkt werden. Die Abfuhr von Schad- bzw. Geruchsstoffen, die z.B. aus Baumaterialien und Einrichtungsgegenständen austreten oder durch menschliche Aktivitäten entstehen, wird durch das Lüften ebenfalls sichergestellt. Ein ausreichender Luftwechsel soll den Frischluftbedarf der sich im Gebäude aufhaltenden Menschen gewährleisten. Mit dem Lüften geht einher, dass in der Heizperiode kalte Umgebungsluft in das Gebäude gelangt und diese aufgeheizt werden muss. Um den Energieverbrauch hierfür möglichst gering zu halten, sollte das Lüften aus energetischen Gründen kontrolliert und nach Bedarf erfolgen.

Einschlägige Normen und Verordnungen behandeln den jeweils zu berücksichtigenden Luftwechsel unterschiedlich. Der planmäßige Abluftvolumenstrom ist in DIN 1946-6:1994-09 [1] bei freier Lüftung mit 60 bzw. $120 \mathrm{~m}^{3} / \mathrm{h}$ für Wohnungsgrößen von $\leq 50 \mathrm{~m}^{2}$ (bis zwei Personen) bzw. $>80 \mathrm{~m}^{2}$ (bis sechs Personen) angegeben. In E DIN 4701-1:1995-08 [2] wird bei der Wärmebedarfsrechnung von einem Mindestluftwechsel von $0,5 \mathrm{~h}^{-1}$ ausgegangen. Die Energieeinsparverordnung (EnEV) [7] geht bei freier Fensterlüftung von einem 0,7-fachen Luftwechsel aus, der auf 0,6 reduziert werden kann, wenn ein Dichtheitsnachweis nach DIN EN 13829:200102 [5], Verfahren A, erbracht wird und der erzielte $n_{50}$-Wert $\leq 3,0 \mathrm{~h}^{-1}$ beträgt. Der $\mathrm{n}_{50}$-Wert gibt die Luftwechselrate an, die bei einer Druckdifferenz von 50 Pa über die Gebäudehülle herrscht. Der Mindestluftwechsel ist gemäß DIN 4108-2:2001-03 [4] aus Gründen der Hygiene, der Begrenzung der Raumluftfeuchte sowie ggf. der Zuführung von Verbrennungsluft nach bauaufsichtlichen Vorschriften mit $0,5 \mathrm{~h}^{-1}$ in der Planung sicherzustellen.

Obwohl ein Mindestluftwechsel erforderlich ist, sollte dies nicht dazu führen, dass die Gebäudehülle undicht ausgeführt wird. Weist die Gebäudehülle Leckagen auf, findet über diese ein unkontrollierter Luftaustausch in Abhängigkeit von den meteorologischen Bedingungen zwischen Raum- und Außenluft statt. Dieser Austausch führt zu Energieverlusten, evtl. zu Behaglichkeitseinbußen und ggf. zu Feuchteschäden. Die Feuchteschäden in der Konstruktion sind in den überwiegenden Fällen auf den Feuchtetransport infolge Konvektion von innen nach außen durch Leckagen zurückzuführen [28]. 
Bei natürlich belüfteten Gebäuden erfolgt die Lüftung über das Öffnen und Schließen von Fenster und Türen. Die Effektivität der Lüftung ist u.a. abhängig von

- der Temperaturdifferenz zwischen innen und außen

- der Windgeschwindigkeit, -richtung und -fluktuation

- Anordnung des Fensters in der Fassade

- der Größe der Öffnungsfläche des Fensters

- der horizontalen und vertikalen Anordnung der Öffnungsflächen

- der Laibungstiefe und -abstand zum Fenster

- der Anordnung eines Heizkörpers zum Fenster

- dem Fensterschmuck, z.B. Pflanzen, Gardinen

- dem Lüftungsverhalten des Nutzers

Die Aufzählung zeigt, dass die Lüftungseffektivität von vielfältigen Faktoren beeinflusst wird. Auf einige dieser Faktoren kann der Nutzer einwirken, jedoch sind die meisten bauseits festgelegt bzw. von den vorherrschenden meteorologischen Bedingungen abhängig.

Zur Bestimmung des Luftwechsels muss der Zu- oder Abluftvolumenstrom, der in ein Gebäude oder einen Raum eindringt bzw. austritt, bestimmt werden. Bezieht man den Volumenstrom auf das Gebäude- oder Raumvolumen, ergibt sich eine gebäude- bzw. raumspezifische Luftwechselrate. In der vorliegenden Arbeit wird der Luftwechsel mit Hilfe des in den Raum eintretenden Volumenstroms, d.h. dem Zuluftvolumenstrom, bestimmt. Für den entsprechenden Massenstrom muss der Zuluftvolumenstrom mit der Dichte der Außentemperatur multipliziert werden.

\subsection{Antriebskräfte}

Die treibenden Kräfte für den Luftwechsel bei einer natürlichen Lüftung sind die über die Öffnungsfläche der Tür oder des Fensters anliegende Temperaturdifferenz und der lokale Wind. Beide Effekte erzeugen einen Druckunterschied zwischen innen und außen, der zu einer Luftbewegung führt. In Bild 1 sind die Effekte schematisch dargestellt.

Auf der Luvseite des Gebäudes wird der Wind gestaut, und es baut sich gegenüber dem statischen Druck in der ungestörten Strömung - Referenzströmung - ein Überdruck auf. Leeseitig entsteht entsprechend ein Unterdruck. Befinden sich Leckagen oder geöffnete Fenster auf beiden Seiten des Gebäudes, entsteht infolge der Sog- und Druckwirkung des Windes eine Luftströmung durch das Gebäude. Die an der Fassade anliegenden Drücke sind abhängig von der Windgeschwindigkeit und 

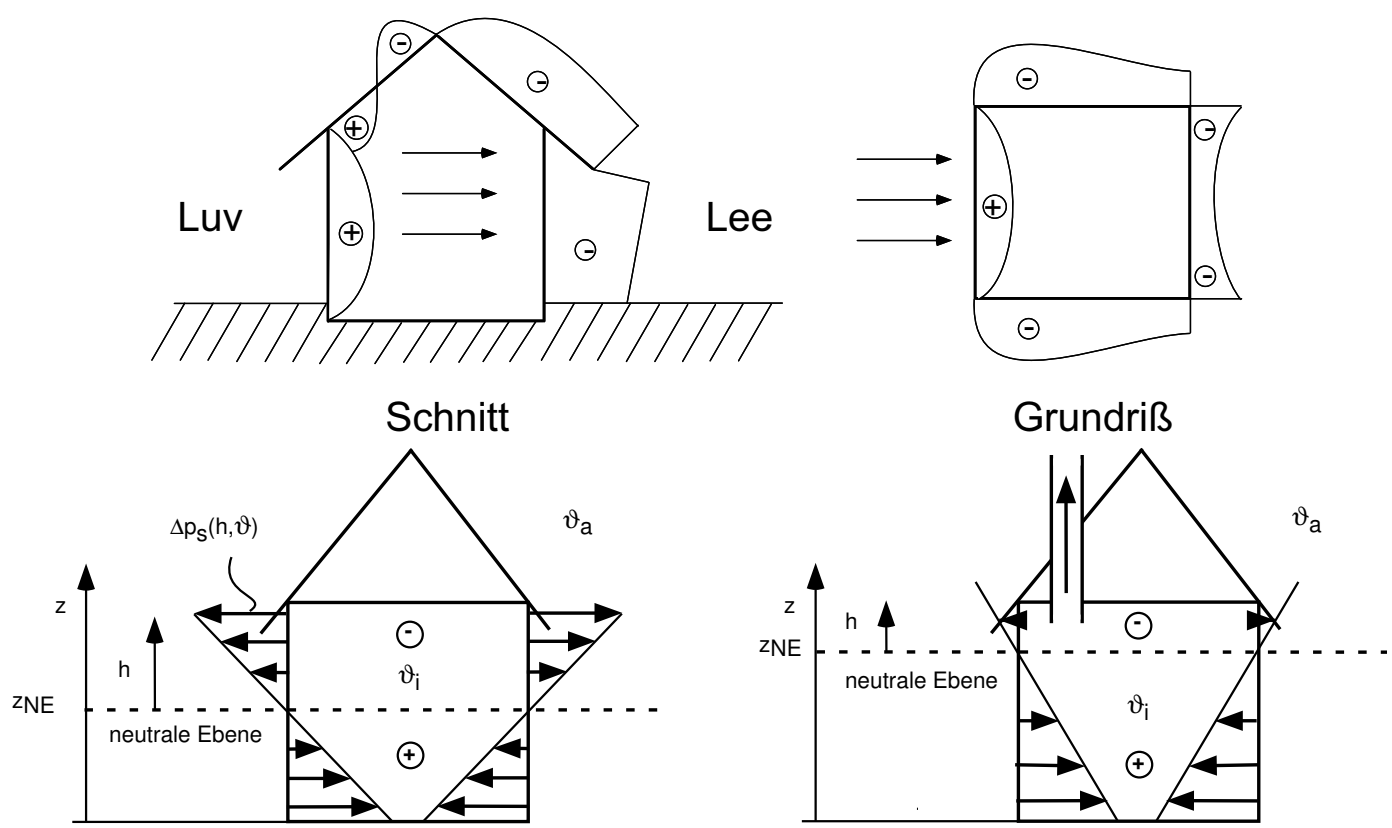

Bild 1: Wind- und Temperatureinfluss.

-richtung, der Böigkeit des Windes, von der umliegenden Geländestruktur und Bebauung sowie von der Fassade selbst. Diese strömungsbedingten Druckdifferenzen entlang der Gebäudehülle werden durch sogenannte Winddruckbeiwerte $c_{p}$ ausgedrückt. Sie sind definiert als das Verhältnis von der Differenz des Druckes an einer Stelle der Fassade und dem statischen Druck in der Referenzströmung zu dem dynamischen Druck in der Referenzströmung

$$
c_{p}=\frac{\Delta p}{1 / 2 \cdot \rho \cdot u^{2}} \quad[-]
$$

Die lokale meteorologische Windgeschwindigkeit u muss i.d.R. aus allgemeinen Klimadaten abgeleitet und auf eine Referenzhöhe bezogen werden [30, 10, 26]. Übliche Referenzhöhen an Gebäuden sind die First- oder Traufhöhe [63].

Die genaue Evaluation der Winddruckbeiwerte ist eine der schwierigsten Aufgaben bei der Modellierung von Infiltration und Lüftung und ist bisher nicht alleine aus theoretischen Betrachtungen abzuleiten [30]. Daher wird in vielen Fällen die Winddruckverteilung am Gebäude experimentell im Windkanal oder mit FeldMessungen bestimmt. Zusammenstellungen von Literaturwerten für Winddruckbeiwerte aus Windkanal-Untersuchungen bzw. Feld-Messungen können z.B. [24, 9] und [36] entnommen werden. Die Berechnung von $\mathrm{c}_{\mathrm{p}}$-Werten kann mit Computational Fluid Dynamics (CFD)-Programmen oder z.B. mit dem Programm Cpcalc [25] erfolgen. In [58] wird jedoch darauf hingewiesen, dass der Einsatz von Cpcalc nur mit eingeschränkten Randbedingungen zu einer guten Übereinstimmung mit Ergebnissen aus Windkanal-Untersuchungen führt.

Die Temperaturdifferenz zwischen innen und außen erzeugt eine Druckdifferenz über die Gebäudehülle, die eine Luftströmung, z.B. über Leckagen, initiiert. Zwei Druckverteilungen über die Fassade in Abhängigkeit von der Leckageverteilung sind exemplarisch in Bild 1b dargestellt. Im oberen Bereich des Gebäudes strömt 
die warme Luft nach außen, und im unteren Teil strömt die kalte Umgebungsluft in das Gebäude ein. In Abhängigkeit von der Höhe ergibt sich ein lineares Druckprofil. Bei einer gleichmäßigen Leckageverteilung wird in der Mitte des Gebäudes die anliegende Druckdifferenz 'Null'. Unterhalb der sogenannten neutralen Höhe/Ebene strömt die Luft in das Gebäude ein, oberhalb aus dem Gebäude aus. Damit ergibt sich für die ein- oder ausströmende Luft die Druckdifferenz

$$
\Delta p=\Delta \rho \cdot g \cdot h
$$

als Antriebskraft. 


\subsection{Bestimmung des Luftwechsels}

Die Tracergas-Technik ist ein übliches Verfahren, um Luftwechselmessungen an Gebäuden bzw. in einzelnen Räumen durchzuführen. Für die Bestimmung des Zubzw. Abluftvolumenstroms eines betrachteten Raumes wird das Tracergas in diesen eingebracht. Die Tracergaskonzentration bzw. die zugeführte Tracergasmenge wird als Funktion der Zeit bestimmt, und mit Hilfe eines mathematischen Auswerteverfahrens wird der $\mathrm{Zu}$ - bzw. Abluftvolumenstrom ermittelt. Der gesuchte Luftwechsel ergibt sich aus dem Verhältnis des ermittelten Volumenstroms und dem Volumen des untersuchten Raumes / Gebäudebereiches. Hierzu ist der Einsatz der Konzentrationsabfall-Methode, der Konstant-Methode oder der Konstantkonzentrations-Methode üblich [10, 37, 6]. Das eingesetzte Tracergas muss bestimmte Kriterien erfüllen [15]. Es darf u.a. weder gesundheitliche Schäden bei Personen hervorrufen noch Materialien angreifen. Das Gas muss auf jeden Fall ungiftig, nicht brennbar und nicht explosiv sein. Ein Absorption des Gases durch im Raum vorhandene Materialien ist zu vermeiden. In der Vergangenheit wurden Helium, Wasserstoff, Methan, Kohlenmonoxid und -dioxid, Distickstoffoxid und sogar radioaktive Gase verwendet [46]. Üblich sind die Gase Schwefel-Hexafluorid $\left(\mathrm{SF}_{6}\right)$ und Distickstoffoxid $\left(\mathrm{N}_{2} \mathrm{O}\right)$. Sie sind beide nach [35] gleichermaßen geeignet.

Während einer Tracergas-Untersuchung ist die gleichmäßige Verteilung des Gases im Raum für die gesamte Messdauer wichtig. Eine vollständige Durchmischung liegt dann vor, wenn zu jeder Zeit und an jedem Punkt im Raum die Konzentration gleich ist. Eine unvollständige Durchmischung würde bei gleichzeitiger Messung an verschiedenen Stellen im Raum zu unterschiedlichen Luftwechseln führen. Wie hoch der mittlere Luftwechsel für den gesamten Raum ist, bleibt in diesem Fall unklar.

In der Realität ist die Ermittlung des Luftwechsels stark von sich ändernden, nicht vorhersehbaren und z.T. nicht beeinflussbaren Randbedingungen abhängig, wie u.a. von der Windgeschwindigkeit, -richtung, Innen- und Außenlufttemperaturen und den Temperaturen von benachbarten Räumen. Undichtheiten in der Gebäudehülle sowie zu benachbarten Räumen oder Gebäudebereichen beeinflussen ebenfalls den Luftwechsel. Die Reproduzierbarkeit von Messergebnissen ist nicht jederzeit gegeben. Infolge Einzelmessungen an realen Objekten können keine allgemeingültigen Aussagen über den Luftwechsel getroffen werden [37].

Zur Abschätzung der Raumluftqualität ist die Kenntnis des Luftwechsels nur bedingt geeignet, da keinerlei Aussagen über lokale Luftströmungen - zeitliche und räumliche Verteilung der Luft - getroffen werden können. Die Effizienz des Luftaustausches in unterschiedlichen Bereichen eines untersuchten Raumes lässt sich nicht ableiten. In [46] wird deshalb auch die Ermittlung des globalen Luftwechsels als Durchschnittswert für Räume oder gar ganze Gebäudebereiche in Frage gestellt.

Trotz der o.a. Probleme stellt die Tracergas-Technik momentan die einzige praktisch verfügbare Möglichkeit dar, den natürlichen Luftwechsel an einem realen Raum oder Gebäude vor Ort zu bestimmen [37].

Die im Rahmen dieser Arbeit durchgeführten Labormessungen erfolgen nach dem 
Prinzip der Konzentrationsabfall-Methode. Der Zuluftvolumenstrom wird nach [37] aus

$$
\dot{V}_{z u}=\frac{T_{z u}}{T_{i}} \frac{V_{R}}{\Delta t} \ln \frac{c(0)}{c(\Delta t)} \quad\left[m^{3} / h\right]
$$

bestimmt. Mit einem bekannten Raumvolumen $\mathrm{V}_{\mathrm{R}}$ wird die gesuchte Luftwechselrate $\mathrm{n}$ durch den Zusammenhang

$$
n=\frac{\dot{V}_{z u}}{V_{R}} \quad\left[h^{-1}\right]
$$

ermittelt.

\subsection{Fensterlüftung in der Literatur - ein Überblick}

In der Literatur sind experimentelle Untersuchungen zur einseitigen natürlichen Lüftung an realen Fenstern bzw. rechteckigen Öffungen in z.B. [22, 29, 14] im Maßstab 1:1 unter Laborbedingungen sowie unter Realbedingungen in z.B. [37, 17, 18, 16, 59, 11] zu finden, wobei nur [37, 29, 14, 16, 59, 11] explizit auch bzw. nur Kippfenster betrachtet haben. Die treibende Kraft für den Luftwechsel variiert bei den Untersuchungen zwischen einem reinen thermischen Antrieb unter Laborbedingungen und einer Kombination aus thermisch- und windinduzierten Effekten an Realräumen, wobei z.B. in [16] darauf hingewiesen wird, dass die Messungen nur bei Windgeschwindigkeiten kleiner $1,5 \mathrm{~m} / \mathrm{s}$ erfolgten.

In [22] werden die Ergebnisse einer zweidimensionalen CFD-Simulation und eines einfachen Massenflussmodells Messergebnissen für eine einseitige Lüftung über eine rechteckige Öffnungsfläche an einem realen Gebäude gegenübergestellt. Die Windgeschwindigkeit wird als 'vernachlässigbar klein' angegeben. Die Experimente und Rechnungen zeigen, dass die einfallende Luft einen schnellen Temperaturabfall im Raum bewirkt, bis ein stationärer Zustand in Abhängigkeit von den Oberflächentemperaturen der Raumumschließungsflächen, den Möbeln und der Heizung erreicht ist. Dieser Zustand stellt sich nach ca. 2 - 3 Minuten ein. Der vertikale Temperaturgradient im Raum liegt bei rund $4 \mathrm{~K}$. In dem Bereich oberhalb des Fenster kann nur eine geringe Temperaturänderung während der Lüftungszeit festgestellt werden, so dass angenommen wird, dass dieser Bereich während der Lüftung kaum beeinflusst wird. Messung und Rechnung können auf Grund verschiedener Randbedingungen nicht miteinander verglichen werden, da die Messungen erst nach den CFD-Simulationen erfolgten.

Labormessungen werden in [29] an einem Dreh- und einem Kippfenster beschrieben. Im Vergleich der beiden Fenstertypen wird bei einseitiger Fensterlüftung und Querlüftung das Lüften über das Kippfenster als 'angenehmer' erachtet, da die kalte Luft seitlich des Fensters und damit nicht direkt in den Aufenthaltsbereich einströmt. Die messtechnischen Ergebnisse zeigen, dass der Discharge-Koeffizient mit der Öffnungsfläche, dem Fenstertyp und der anliegenden Temperaturdifferenz variiert. 
In [17] wird die einseitige Lüftung durch 52 verschiedene Kombinationen aus rechteckigen Öffnungen messtechnisch untersucht und mit den Ergebnissen aus sechs Mehrzonen-Strömungsprogrammen verglichen. Alle Programme weisen für überwiegend windinduzierte Luftwechsel systematische Abweichungen von den Messwerten auf, die je nach Randbedingungen positiv oder negativ sind. Ein einheitlicher Korrekturfaktor wird für alle sechs Programme bestimmt.

Eine kombinierte Messung der Luftgeschwindigkeit und des Luftwechsels über eine Tür an einem realen Testraum werden in [18] beschrieben. Die Luftgeschwindigkeit wird in der Mitte der Türöffnung in fünf verschiedenen Höhen bestimmt. Die Ermittlung des Luftwechsels erfolgt mit der Tracergas-KonzentrationsabfallMethode. Die Windgeschwindigkeit liegt bei den Messungen zwischen 1,5 m/s und $2,0 \mathrm{~m} / \mathrm{s}$. Die Temperaturdifferenz beträgt $1 \mathrm{~K}$ bis $3 \mathrm{~K}$. Vergleichende Rechnungen werden mit einem Mehrzonen-Strömungsprogramm durchgeführt. Die vorhergesagten Luftgeschwindigkeiten im Bereich der Türöffnung liegen deutlich unter den gemessenen Geschwindigkeiten und weisen eine homogene Geschwindigkeitsverteilung auf, welche in der Realität nicht vorliegt. Der berechnete Luftwechsel stimmt jedoch gut mit den Ergebnissen der Tracergas-Messung überein.

Übersichten über Modellansätze zur Bestimmung des Zuluftvolumenstroms für rechteckige Fensteröffnungen sind z.B. in [9] und [56] zu finden.

Messtechnische Untersuchungen zu Kippfenstern in Doppelfassaden werden in [40] und [62] beschrieben. Während in [40] nur Messungen an einem realen Bürogebäude durchgeführt werden, erfolgen in [62] auch Untersuchungen im Labor.

Das Querlüftungspotential von zwei gegenüberliegenden Kippfenstern wird unter realen Bedingungen in [38] untersucht. Die Messwerte belegen, das bei rein thermisch bedingtem Luftaustausch die Modelle für einseitige Fensterlüftung auch für eine Querlüftung gültig sind. Wirken Wind und Temperaturdifferenz gleichzeitig, ist der Einfluss der Temperaturdifferenz auf den Luftwechsel bei Querlüftung zu vernachlässigen.

Da weder die einseitige Lüftung von Kippfenstern in einer Doppelfassade noch die Querlüftung über zwei gegenüberliegende Kippfenster Gegenstand der vorliegenden Arbeit sind, wird hierauf im weiteren nicht eingegangen.

In den Veröffentlichungen von [34] und [52] werden messtechnische und rechnerische Zuluftvolumenströme über Kippfenster betrachtet. Die Autoren greifen jedoch auf die Ansätze von [37] und [16] zurück, die in den Abschnitten 3.3 und 3.6 ausführlich erläutert werden, so dass [34] und [52] an dieser Stelle nicht weiter zu präzisieren sind.

Verschiedene Ansätze zur Beschreibung des Luftwechsels über Kippfenster bei einseitiger Lüftung werden in $[37,16,59,11,57]$ beschrieben und im folgenden Kapitel 3, nach einer allgemeinen Betrachtung eines Kippfensters, vorgestellt. 


\section{Thermisch induzierter Luftwechsel über Kippfenster}

\subsection{Allgemeines}

In dieser Arbeit sollen die Einflüsse von verschiedenen Randbedingungen auf den thermisch induzierten Luftwechsel über Kippfenster bestimmt werden. Der Windeinfluss wird aus folgenden Gründen nicht berücksichtigt:

- Windstille ist ein natürlicher meteorologischer Zustand. Daher entspricht die Untersuchung des Luftwechsels ohne Windeinfluss realen Bedingungen.

- Bei zunehmendem Wind wird das Fenster entsprechend schnell geschlossen, weil Zugerscheinungen auftreten. In diesem Fall findet kein weiterer Luftaustausch statt und eine Betrachtung des Luftwechsels ist nicht erforderlich.

- Der thermisch bedingte Luftwechsel ist i.d.R. geringer als der windinduzierte. Die Betrachtung des thermisch induzierten Luftwechsels stellt also eine Minimumbetrachtung dar. Kenntnisse hierüber sind wichtig, wenn Mindestluftwechsel eingehalten werden müssen.

- Um den Einfluss der verschiedenen Randbedingungen auf den Luftwechsel und den Temperaturverlauf exakt bestimmen zu können, müssen die Randbedingungen für den Luftaustausch bei allen messtechnischen Untersuchungen fest definiert, frei einstellbar und reproduzierbar sein. Diese Forderungen sind an dem vorhandenen Versuchsaufbau nur für Temperaturdifferenzen gegeben.

\subsection{Geometrie eines Kippfensters}

Die Öffnungsfläche eines Kippfensters setzt sich aus zwei Dreiecken und einem Rechteck zusammen. Die Kippachse befindet sich bei einem üblichen Dreh-Kippfenster am Fußpunkt des Fensterflügels. Dadurch ergibt sich, dass zwei Dreiecke - zwischen Flügel und Blendrahmen - vertikale Öffnungen bilden, während das Rechteck - zwischen Oberkante des gekippten Flügels und dem Blendrahmen eine horizontal liegende Öffnung beschreibt. Solch ein Kippfenster ist Gegenstand der vorliegenden Arbeit.

Infolge der üblichen geringen Kippwinkel von Standardfenstern - nur ca. 4 - 7 Grad - und der Tiefe des Flügelrahmens erstrecken sich die seitlichen Dreiecke nicht über die gesamte lichte Höhe H. Die Öffnungshöhe $\mathrm{H}$ verringert sich um einen bestimmten Betrag $\mathrm{x}$, der von der Rahmentiefe $\mathrm{d}$ und der Kippweite s abhängig ist (Bild 2). Die reduzierte Höhe h gibt die tatsächliche Höhe der Dreiecke an, die für den Luftwechsel zur Verfügung steht. Die Höhe x kennzeichnet die Höhe der Rahmenüberschneidung von Blend- und Flügelrahmen. In diesem Bereich ist die Öffnungsfläche nur von der Höhe der Rahmenüberschneidung und dem Abstand zwischen Blendrahmen und Kippflügel abhängig. Der Abstand zwischen den beiden Rahmenteilen beträgt üblicher Weise ca. $1 \mathrm{~cm}$. 


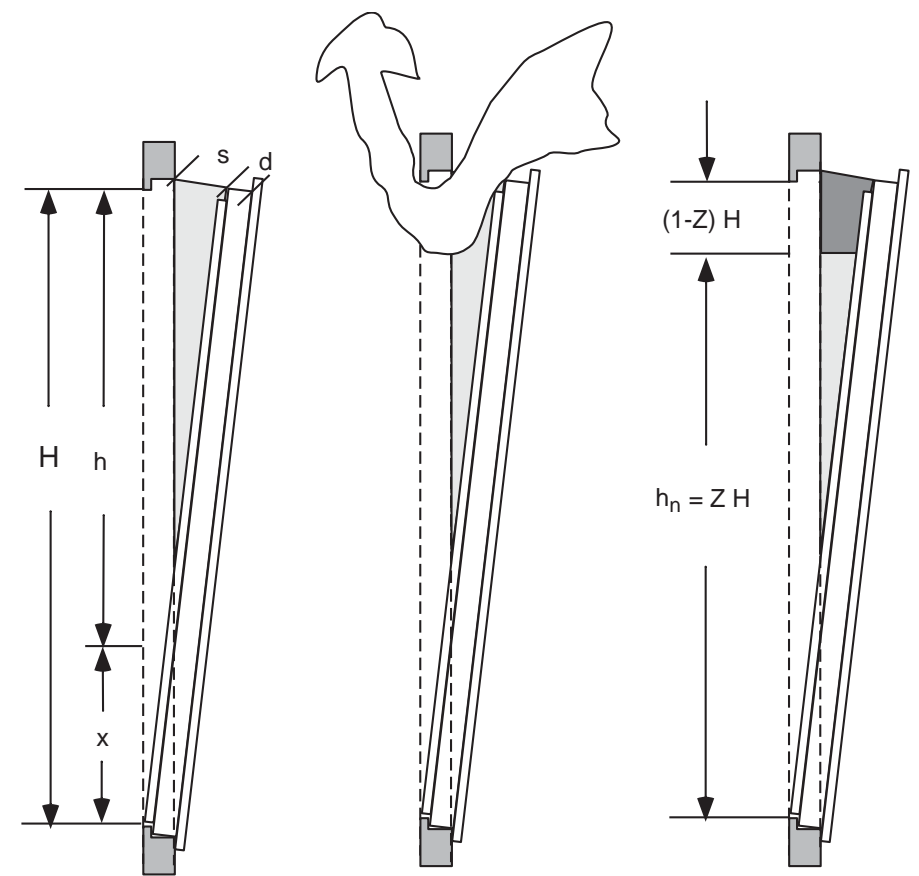

Bild 2: Ein- und Ausströmbereich eines Kippfensters.

Bei der einseitigen Fensterlüftung ist über die Öffnungsfläche die Luftströmung bidirektional. Die Öffnungsfläche des Kippfensters teilt sich daher in einen $\mathrm{Zu}$ und Ausströmbereich. Prinzipiell strömt - bei einem Kippfenster mit der Kippachse am Fußpunkt des Fensters - durch die vertikalen Dreiecke die kalte Luft ein, während die warme Luft durch das horizontale Rechteck am oberen Ende des Fensters austritt. Infolge der ausströmenden Luft steht geometriebedingt der obere Bereich der Dreiecke nicht für einen Zuluftstrom zur Verfügung (Bild 2, Mitte). Es entsteht in der dreieckigen Fläche ein Bereich, in dem der Umschlag von zu- und ausströmender Luft stattfindet. Die entsprechende Fensterhöhe wird neutrale Höhe genannt. Sie wird im folgenden durch den Faktor $\mathrm{Z}$ ausgedrückt, der das Verhältnis von neutraler Höhe $h_{n}$ zur gesamten Fensterhöhe $H$ beschreibt.

$$
Z=\frac{h_{n}}{H} \quad[-]
$$

Für vertikal symmetrische, große Öffnungen, z.B. ein rechteckiges Fenster oder eine Tür, befindet sich die neutrale Höhe, infolge der Dichteunterschiede der einund ausströmenden Luft, in etwa auf der halben Öffnungshöhe [10]. Bei einem Kippfenster liegt eine vertikal asymmetrische Verteilung der Öffnungsfläche vor, so dass in diesem Fall die neutrale Höhe oberhalb der halben Fensterhöhe ist [37, 16]. Auf der linken Seite von Bild 3 ist die einströmende Luft durch Nebel visualisiert. Es ist deutlich zu erkennen, dass die neutrale Höhe oberhalb der halben Fensterhöhe liegt. Auf dem rechten Bild ist die ausströmende Luft im Bereich des Rechteckes zu sehen.

Zur Verdeutlichung der Strömung durch die Öffnungsfläche eines Kippfensters sind in Bild 4 die mittleren Geschwindigkeitsvektoren in der Öffnungsfläche aus einer 

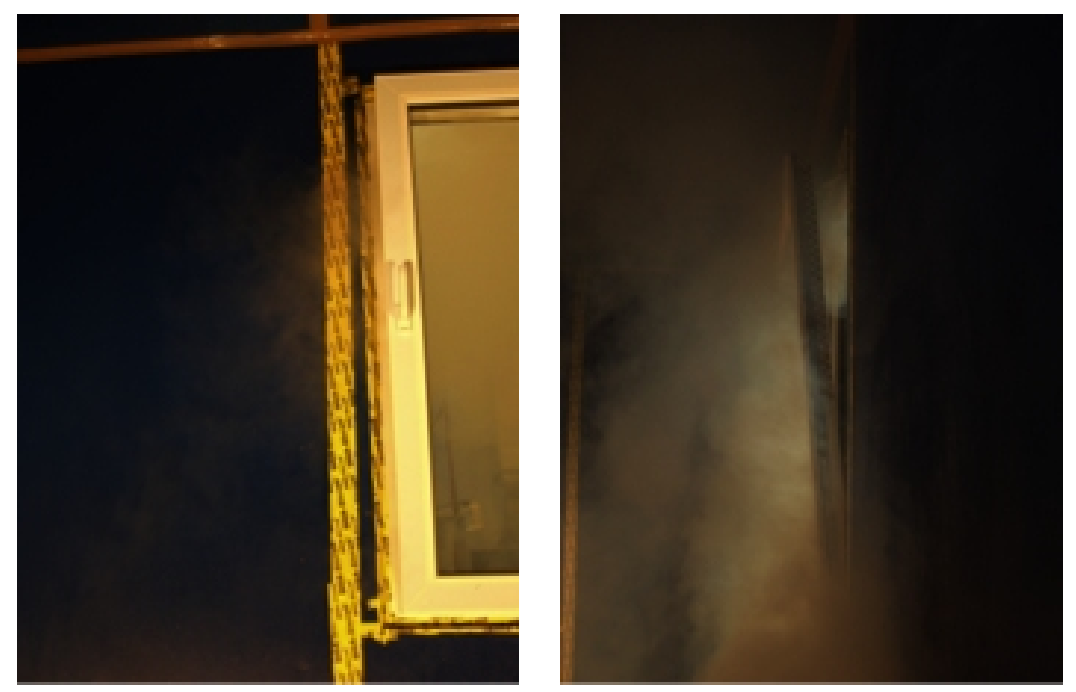

Bild 3: Visualisierung der ein- und ausströmenden Luft an einem Kippfenster.

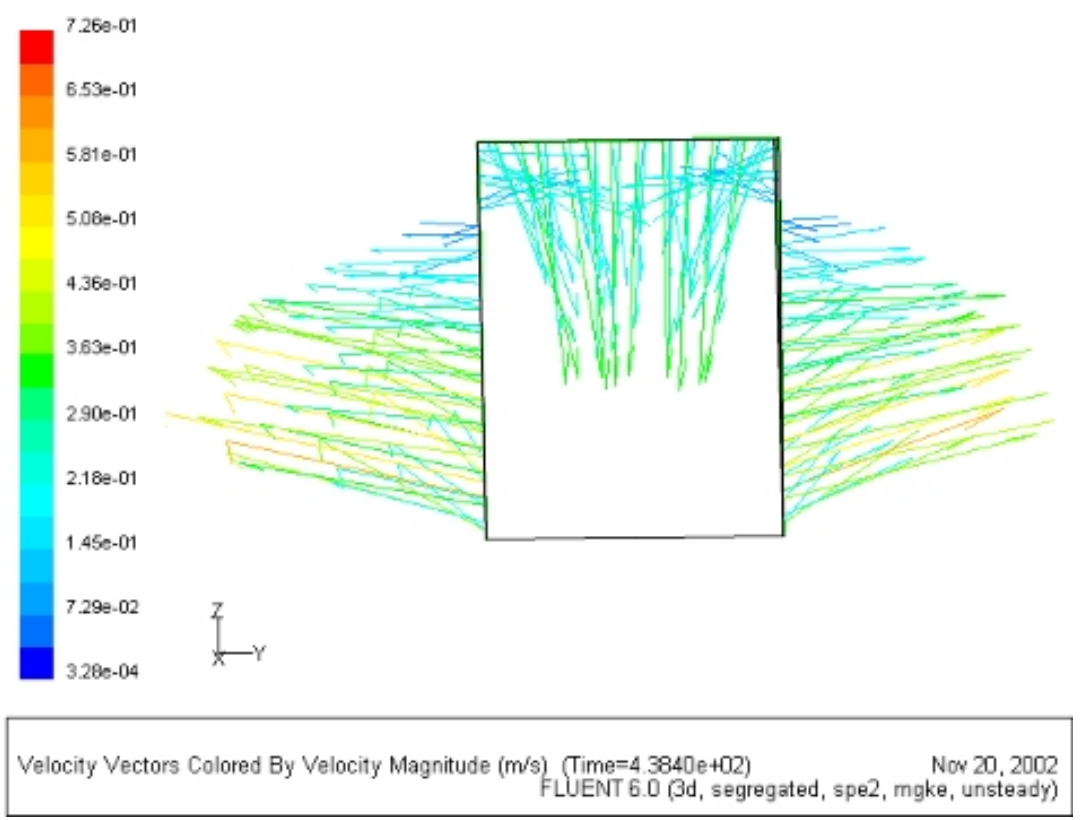

Bild 4: Geschwindigkeitsvektoren an der Öffnungsfläche eines Kippfensters (Frontalansicht). 
Strömungssimulation als Momentaufnahme dargestellt. Das Bild zeigt eine Frontalansicht auf ein Kippfenster. Die unterschiedlichen Farben der Strömungpfeile sowie die Pfeillängen repräsentieren verschiedene Strömungsgeschwindigkeiten. Die Pfeilrichtung zeigt die Strömungsrichtung an.

In dieser Darstellung ist zu erkennen, dass durch die seitlichen Öffnungsflächen eine bidirektionale Strömung anliegt. Deutlich zeichnet sich die neutrale Höhe im oberen Bereich der Dreicke ab. Unterhalb der neutralen Höhe strömt die Luft in den Raum ein, wie es auch auf der linken Seite von Bild 3 zu sehen ist. Oberhalb der neutralen Höhe strömt die Luft aus dem Raum aus. Die Pfeile, die vom oberen horizontalen Rahmen nach unten zeigen, stellen die Strömungsrichtung und -geschwindigkeit der Luft dar, die durch das horizontale Rechteck den Raum verlässt. Das Rechteck wird nur in eine Richtung durchströmt.

Fensterrahmen schließen i.d.R. nicht bündig mit der raumseitigen Bekleidung ab, sondern werden in die Dämmschicht integriert, d.h. die Anordnung erfolgt mehr auf der Außenseite der Konstruktion. Damit geht einher, dass eine gewisse Laibungstiefe vorliegt (Bild 5). I.d.R. beträgt der Abstand zwischen Laibung bzw. Sturz / Fensterflügel nur wenige $\mathrm{cm}$. Durch die Laibungstiefe 1 und die Laibungsabstände $\mathrm{j}$ und $m$ wird in den meisten Fällen die effektive Öffnungsfläche reduziert. Der engste Querschnitt ist für die ein- und ausströmende Luft maßgeblich, so dass die Berechnung der effektiven Austauschfläche bei Fenstern mit Laibung anders vorgenommen werden muss als bei Fenstern ohne Laibung.
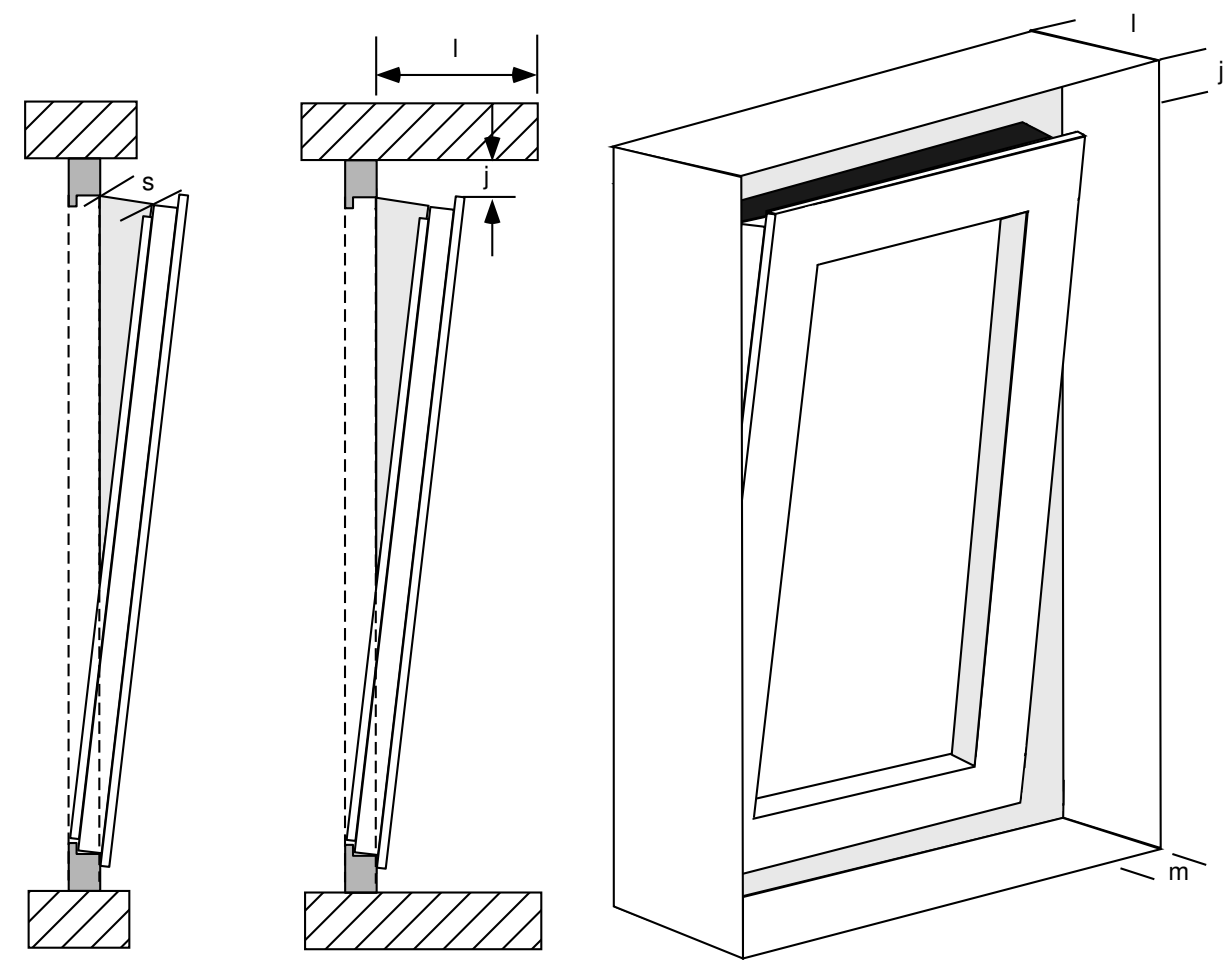

Bild 5: Kippfenster mit Laibung. 


\subsection{Ansatz Daler}

In [16] wird für den Zu- bzw. Abluftvolumenstrom über ein Kippfenster ein Ansatz aus theoretischen und praktischen Untersuchungen hergeleitet

$$
\dot{V}_{D}=496 \alpha f_{D} s Z^{5 / 2} H^{3 / 2} \sqrt{\Delta T} \quad\left[m^{3} / h\right]
$$

Durch die empirische Durchflusszahl $\alpha$ ist die Abminderung der Öffnungsfläche durch Beschläge, die Schere bei kleinen Kippstellungen und ähnliche Rahmeneinflüsse pauschal berücksichtigt. Der Faktor $f_{D}$ beschreibt die Abweichung der dreieckigen seitlichen Öffnungsflächen von der Idealform eines Dreieckes infolge der Falzausbildung.

An einem Dreh-Kippfenster mit den Maßen 1,2 m x $1 \mathrm{~m}(\mathrm{H}$ x B) werden mit dem Tracergas Methan $\left(\mathrm{CH}_{4}\right)$ Untersuchungen unter natürlichen Randbedingungen durchgeführt. Zur Beheizung des Raumes $\left(\mathrm{V}=28,4 \mathrm{~m}^{3}\right)$ dienen drei geregelte Elektroradiatoren, wobei sich einer direkt unter dem untersuchten Fenster befindet und die anderen beiden Radiatoren jeweils unter weiteren Fenstern, welche nicht Gegenstand der Untersuchung sind. Der Blendrahmen des betrachteten Fensters schließt bündig mit der Innenseite der Außenwand ab, d.h. es ist keine Laibung vorhanden.

Messungen und Berechnungen ergeben für das untersuchte Fenster von Daler, dass der Faktor $f_{D}=0,7$ zwischen Öffnungsweiten von $2 \mathrm{~cm}$ bis $12 \mathrm{~cm}$ als konstant betrachtet werden kann. Aus den praktischen Untersuchungen wird die Durchflusszahl zu $\alpha=0,85$ ermittelt.

Der Faktor Z zur Bestimmung der neutralen Höhe muss über Gleichung 7 iterativ bestimmt werden. Sie häng nach Daler nur von der Fensterhöhe und -breite ab.

$$
\frac{B}{H f_{D}}=\frac{4}{15}\left(\frac{2 Z^{5 / 2}}{\sqrt{1-Z}}+2 Z^{2}+Z-3\right)
$$

Der Ansatz von Daler wird aus Messwerten, die bei Windgeschwindigkeiten kleiner $1,5 \mathrm{~m} / \mathrm{s}$ aufgenommen werden, bestimmt, d.h. der Luftaustausch erfolgt fast ausschließlich infolge des Temperaturunterschiedes zwischen innen und außen. Untersucht wurden Kippweiten zwischen $2 \mathrm{~cm}$ und $12 \mathrm{~cm}$. Es wird eine durchschnittliche Abweichung der Messwerte vom Modellansatz von $\pm 28 \%$ angegeben.

\subsection{Ansatz Weberl}

Der Ansatz Weberl [57] zur Bestimmung des Zu- resp. Abluftvolumenstroms durch ein Kippfenster wird mit der Annahme, dass die neutrale Höhe auf der halben Fensterhöhe liegt, wie folgt beschrieben

$$
\dot{V}_{W 1}=C_{d} C_{k}(\varphi) H B \frac{1}{3} \sqrt{\frac{\Delta T}{T_{m}} g H} \quad\left[m^{3} / h\right]
$$


Mit dem $\mathrm{C}_{\mathrm{d}}$-Wert wird der Einfluss der Reibung berücksichtigt und zu 0,68 konstant gesetzt. $\mathrm{T}_{\mathrm{m}}$ gibt die mittlere Lufttemperatur zwischen innen und außen an.

Der Ansatz, ursprünglich für eine rechteckige Fensteröffnung entwickelt, wird über die Korrekturfunktion

$$
C_{k}(\varphi)=2,610^{-7} \varphi^{3}-1,1910^{-4} \varphi^{2}+1,8610^{-2} \varphi \quad[-]
$$

in Abhängigkeit von dem Kippwinkel $\varphi$ an ein Kippfenster angepasst. Untersucht werden unterschiedliche Kippstellungen $\left(\approx 8^{\circ}-180^{\circ}\right)$. Der Ansatz Weberl gilt nur für den thermisch induzierten Luftaustausch. Dieser Ansatz ist eine Weiterentwicklung des Ansatzes aus dem Multizonen-Luftströmungsprogramm Comis 3.0 [20].

\subsection{Ansatz Weber2}

Eine weitere Beziehung zur Berechnung des Volumenstromes über ein Kippfenster wird in einem zweiten Ansatz von Weber, ebenfalls in [57], vorgestellt. Sie lautet

$$
\dot{V}_{W 2}=C_{d}(\varphi, H, B) H^{2} \tan (\varphi) Z^{5 / 2} \frac{8}{15} \sqrt{\frac{\Delta T}{T_{m}} g H} \quad\left[m^{3} / h\right]
$$

Durch die Funktion

$$
C_{d}(\varphi, H, B)=0,0147 \varphi-0,0928 \frac{H}{B}+0,4116 \quad[-]
$$

wird der Einfluss der Reibung in Abhängigkeit von dem Kippwinkel, der Fensterhöhe und -breite berücksichtigt. Der Faktor Z zur Bestimmung der neutralen Höhe des Fensters muss iterativ bestimmt werden. Für $\mathrm{Z} \leq \cos (\varphi)$ gilt

$$
4 Z^{\frac{5}{2}}-2[\cos (\varphi)-Z]^{\frac{3}{2}}[3 \cos (\varphi)+2 Z]=\frac{5 B\left[(1-Z)^{\frac{3}{2}}-(\cos (\varphi)-Z)^{\frac{3}{2}}\right]}{H \tan (\varphi)}
$$

Die experimentellen Untersuchungen zur Bestimmung der $\mathrm{C}_{\mathrm{d}}(\varphi, H, B)$-Funktion werden in einem Labor durchgeführt und mit CFD-Rechnungen verglichen [14]. Ein Modellraum $\left(\mathrm{V}=76 \mathrm{~m}^{3}\right)$ wird durch eine Trennwand in zwei gleich große Räume - Kalt- und Warmseite - unterteilt. Das untersuchte Fenster mit der Größe $1,14 \times 0,78 \mathrm{~m}(\mathrm{H} \times \mathrm{B})$ ist mittig und bündig, d.h. ohne Laibung, in die Trennwand eingebaut. Die gewünschten Temperaturdifferenzen werden über wasserführende Metallpanele in der Decke, dem Boden und zwei Wandflächen des Modellraumes eingestellt. Die Untersuchungen erfolgen an den Kippweiten von 8, $6^{\circ}-18,7^{\circ}$. Der Ansatz ist lt. [57] 'für gängige Verhältnisse der Fensterhöhe zur Fensterbreite und kleine Kippwinkel bis ca. $25^{\circ}$ brauchbar'.

\subsection{Ansatz Maas}

Der Ansatz Maas für den ausgetauschten Volumenstrom über ein Kippfenster wird durch experimentelle Untersuchungen unter realen meteorologischen Bedingungen $\mathrm{zu}$ 


$$
\dot{V}_{M}=3600 H B \Theta \frac{1}{2} \sqrt{0,0056 u^{2}+0,0037 H \Delta T+0,012}\left[m^{3} / h\right]
$$

bestimmt [37]. Das Durchflussverhältnis $\Theta$ kennzeichnet das Flächenverhältnis von Öffnungsfläche des gekippten Fensters bezogen auf die lichte Fensteröffnung. Es wird die halbe effektive Öffnungsfläche in Ansatz gebracht. Dies entspricht der Annahme, dass die Luft durch jeweils eine Hälfte der Öffnung ein- bzw. ausströmt. Der erste Term unter der Wurzel berücksichtigt den Windeinfluss, der zweite die Temperaturdifferenz zwischen innen und außen und der dritte Term fasst turbulente Effekte zusammen.

Das von Maas untersuchte Fenster weist die lichten Öffnungsmaße von 1,12 m x $0,82 \mathrm{~m}(\mathrm{H} \mathrm{x} \mathrm{B})$ auf. Es werden u.a. Untersuchungen mit und ohne Fensterlaibung durchgeführt. Die Beheizung des Messraumes erfolgt durch einen rollbaren Ölradiator, der unterhalb des Fensters angeordnet ist. Zur Sicherstellung der optimalen Durchmischung der Raumluft mit dem kontinuierlich zuströmenden Tracergas infolge der verwendeten Konstant-Konzentrations-Methode laufen während der Messungen vier handelsübliche Tischventilatoren. Der Messraum umfasst ein Volumen von $58,5 \mathrm{~m}^{3}$.

Der Ansatz von Maas ist für o.g. Fenster für Kippweiten zwischen $2 \mathrm{~cm}$ und 14 $\mathrm{cm}$ sowie für Drehstellungen von $5 \mathrm{~cm}, 10 \mathrm{~cm}$ und $15 \mathrm{~cm}$ gültig und berücksichtigt neben den thermischen Effekten auch den Windeinfluss. Die Koeffizienten werden aus den Ergebnissen der verschiedenen Randbedingungen - mit/ohne Anordnung einer Heizung, mit/ohne Anordnung einer raumseitigen $20 \mathrm{~cm}$ tiefen Laibung sowie für unterschiedliche Dreh- bzw. Kippstellungen - gewonnen.

Maas weist darauf hin, das bei hohen Temperaturdifferenzen - ca. $>20 \mathrm{~K}$ - und ohne Windeinwirkung der Luftwechsel zu gering ermittelt wird. Eine weitere Problematik bildet die Ermittlung des Durchflussverhältnisses. Die Bestimmung des Durchflussverhältnisses aus geometrischen Abmessungen führt im Bereich von kleineren Öffnungsweiten zu nicht akzeptablen Abweichungen. Seine Anpassung der Koeffizienten erfolgt mit messtechnisch bestimmten Durchflussverhältnissen.

\subsection{Ansatz Zeidler}

Der Zuluftvolumenstrom über ein Kippfenster wird in [59] durch das Integral

$$
\dot{V}_{Z}=3600 \mu \int_{0}^{h_{n}} A(z) w(z) d z \quad\left[m^{3} / h\right]
$$

beschrieben. Der Einschnürungsfaktor $\mu$ stellt das Verhältnis der tatsächlich vom Zuluftvolumenstrom durchströmten Fläche zur lichten Öffnungsfläche des Kippfensters dar. Er wird zu $\mu=0,33$ abgeschätzt. Die neutrale Höhe $\mathrm{h}_{\mathrm{n}}$ muss über eine Iteration nach Gleichung 15 bestimmt werden. Hierbei wird vereinfachend angenommen, dass der $\mathrm{Zu}$ - und Abluftvolumenstrom gleich groß ist. 


$$
\dot{V}_{z u}=\int_{0}^{h_{n}} A(z) w(z) d z=\int_{h_{n}}^{H} A(z) w(z) d z=\dot{V}_{a b}
$$

Die Ermittlung der Strömungsgeschwindigkeit erfolgt über

$$
w(z)=\sqrt{2 g z \frac{\Delta T}{T}}[\mathrm{~m} / \mathrm{s}]
$$

Eine Funktion zur Berechnung von $\mathrm{A}(\mathrm{z})$ ist nicht angegeben. Da die Angaben zur Bestimmung des Zuluftvolumenstroms nicht vollständig sind, wird der Ansatz Zeidler im weiteren nicht berücksichtigt.

\subsection{Ansatz Asimakopoulos}

In einer Veröffentlichung von Asimakopoulos [11] wird für ein Fenster mit zwei verschiedenen Kippweiten ein Ansatz vorgestellt, der die Luftwechselrate nur in Abhängigkeit von der Temperaturdifferenz beschreibt

$$
\begin{gathered}
n_{10 \mathrm{~cm}}=0,3736 \Delta T+1,41 \quad[1 / h] \\
n_{20 \mathrm{~cm}}=0,1205 \Delta T+0,5 \quad[1 / h]
\end{gathered}
$$

Die beiden Gleichungen werden durch experimentelle und theoretische Untersuchungen für ein Kippfenster mit den Kippweiten von $10 \mathrm{~cm}$ und $20 \mathrm{~cm}$ bestimmt. Die Untersuchungen erfolgen an einem realen Raum mit dem Volumen von $35 \mathrm{~m}^{3}$ unter realen meteorologischen Bedingungen. Die Größe des Fensters wird nicht angegeben. Da der Ansatz keinerlei Anpassungsmöglichkeiten an eine andere Fenstergeometrie bietet, kann er nur für ein konkretes Fenster gültig sein und wird im folgenden nicht weiter betrachtet.

\subsection{Vergleich der Ansätze}

In Tabelle 1 sind die Randbedingungen der Ansätze von Daler, Weber1/2 und Maas zur Berechnung des Zuluftvolumenstroms durch ein Kippfenster zusammengefasst.

Die Zusammenstellung zeigt, dass die Bildung der Ansätze zur Bestimmung des Zuluftvolumenstroms unter sehr verschiedenen Randbedingungen erfolgten. Es werden jeweils Fenster und Versuchsräume im Maßstab 1:1 unter Labor- bzw. Realbedingungen untersucht. Die Fenster von Daler und Weber weisen keine innenliegende Laibung auf. Maas untersucht auch den Einfluss einer raumseitigen Laibung, fasst die Ergebnisse jedoch mit den Varianten 'ohne Laibung' zusammen. Bei allen Ansätzen wird nicht unterschieden, ob zur Aufrechterhaltung der Temperaturdifferenz zwischen innen und außen ein Heizkörper verwendet wird oder nicht.

In Bild 6 sind die Zuluftvolumenströme für ein Kippfenster, berechnet mit den vier vorgestellten Ansätzen, über der Temperaturdifferenz zwischen innen und außen aufgetragen. Die Maße entsprechen dem in der vorliegenden Arbeit untersuchten 
Tabelle 1: Zusammenstellung der Randbedingungen für die Grundlagen der Ansätze Daler, Weber1/2 und Maas.

\begin{tabular}{|c|c|c|c|}
\hline Parameter & Daler & Weber $1 / 2$ & Maas \\
\hline Tracergas & $\mathrm{CH}_{4}$ & $\mathrm{SF}_{6}$ & $\mathrm{~N}_{2} \mathrm{O}$ \\
\hline Dichteverhältnis Gas / Luft [-] & 0,55 & 5,1 & 1,53 \\
\hline Tracergas-Messmethode & Abfall & Abfall & Konst. Konz. \\
\hline Heizung & Radiator & Fläche & Radiator \\
\hline $\begin{array}{l}\text { Aktive Durchmischung der Luft } \\
\text { während der Messung }\end{array}$ & nein & nein & Ventilator \\
\hline Lichte Fensteröffnung $\mathrm{H}$ x B [m] & $1,2 \times 1,0$ & $1,14 \times 0,78$ & $1,12 \times 0,84$ \\
\hline Fensterlaibung innen & nein & nein & ja/nein \\
\hline Messraumvolumen $\left[\mathrm{m}^{3}\right]$ & 28,4 & 38 & 58,5 \\
\hline Messbedingungen & Realraum & Laborraum & Realraum \\
\hline $\begin{array}{l}\text { Untersuchungsbedingungen } \\
\text { meteorol. Bedingungen } \\
\text { Kippweite bzw. -winkel }\end{array}$ & $\begin{array}{l}\Delta \mathrm{T}, \mathrm{u}<1,5 \\
2-12 \mathrm{~cm}\end{array}$ & $\begin{array}{c}\Delta \mathrm{T} \\
\text { bis } 180^{\circ}(\mathrm{W} 1) \\
\text { bis ca. } 25^{\circ}(\mathrm{W} 2)\end{array}$ & $\begin{array}{c}\Delta \mathrm{T}, \mathrm{u} \\
2-14 \mathrm{~cm}\end{array}$ \\
\hline
\end{tabular}

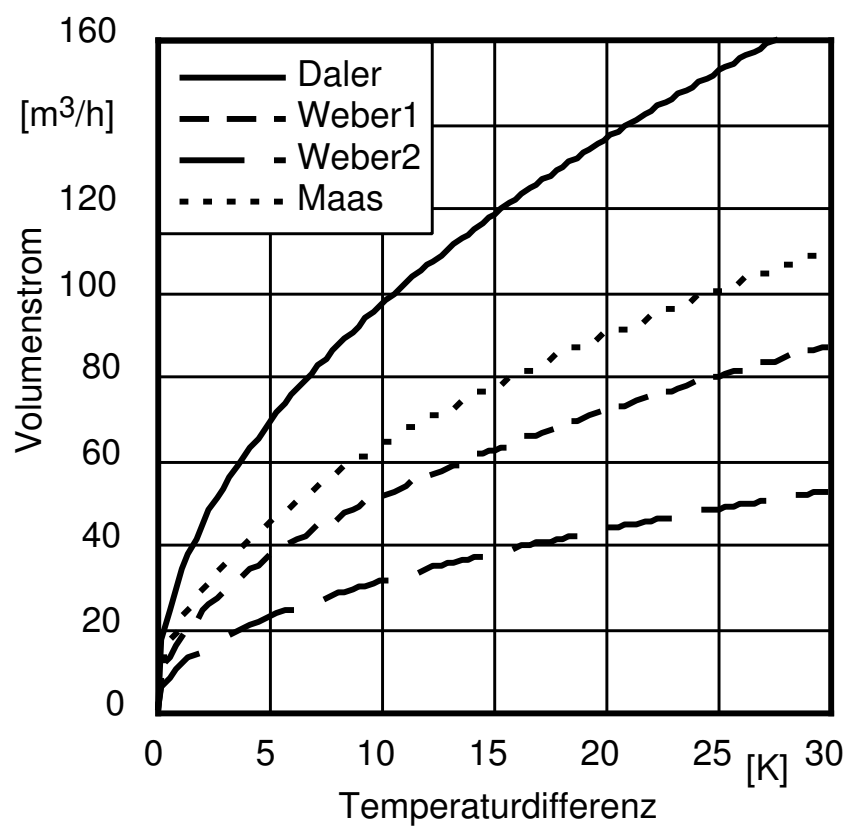

Bild 6: Zuluftvolumenstrom in Abhängigkeit von der Temperaturdifferenz zwischen innen und außen berechnet mit vier verschiedenen Ansätzen (H: 1,23 m, B: 0,94 m, Kippweite $10 \mathrm{~cm}$ ). 
Kippfenster (H x B: 1,23 x 0,94 m, Kippweite $10 \mathrm{~cm}$ ). Es ist eine konstante Innentemperatur von $20{ }^{\circ} \mathrm{Czugrunde}$ gelegt. Im Ansatz Maas werden der Term für den Windeinfluss und der Term für die turbulenten Effekte zu 'Null' gesetzt.

Mit dem Ansatz nach Daler wird der größte und mit dem Ansatz Weber2 der kleinste Zuluftvolumenstrom berechnet. Werden die berechneten Volumenströme nach Weber $2 \mathrm{zu} 100 \%$ gesetzt, so sind die Volumenströme im Vergleich nach Weber1 um den Faktor $\approx 1,6$, nach Maas um den Faktor $\approx 2$ und nach Daler um den Faktor $\approx 3$ größer. Je nach Wahl des Ansatzes kann der ermittelte Luftwechsel bis um den Faktor drei differieren. Die Darstellung zeigt, dass die bekannten Ansätze aus der Literatur zur Beschreibung des Luftwechsels über Kippfenster zu sehr unterschiedlichen Ergebnissen führen und infolge dessen deutliche Differenzen bei der Berechnung der Luftwechselrate und der Lüftungswärmeverluste auftreten. 


\section{Versuchsaufbau}

\subsection{Modellraum und Messtechnik}

Der Modellraum steht in einem doppelstöckigen Labor. Die Abmessungen des Modellraumes betragen 2,5 m x 3,6 m x 5,0 m ( $\mathrm{H} \times \mathrm{B} \times \mathrm{L})$, d.h. der Modellraum verfügt über eine Grundfläche von $18 \mathrm{~m}^{2}$ und umfasst ein Volumen von $45 \mathrm{~m}^{3}$. Das Labor hat die Maße 6,65 m x 6,4 m x 13,3 m (H x B x L), welches einem Volumen von $566 \mathrm{~m}^{3}$ entspricht. Die gesamten Abmessungen und die Anordnung des Modellraumes im Labor sind Bild 7 zu entnehmen. Der Modellraum ist in Holzrahmenbauweise - $6 \mathrm{~mm}$ Sperrholz, $110 \mathrm{~mm}$ extrudierter Polystyrol-Hartschaum $\left(\lambda_{R}=0,035 \mathrm{~W} /(\mathrm{m} \cdot \mathrm{K})\right), 6 \mathrm{~mm}$ Sperrholz - erstellt und weist einen rechnerischen U-Wert von $0,35 \mathrm{~W} /\left(\mathrm{m}^{2} \cdot \mathrm{K}\right)$ auf. Die gesamte raumseitige Oberfläche ist mit AcrylHochglanzlack gestrichen. Der in Bild 7 skizzierte kleine Vorraum ist für die durchgeführten Messungen nicht relevant.

Das untersuchte Fenster ist in der stirnseitigen Fassade des Modellraumes eingebaut. Auf der gegenüberliegenden Seite befindet sich eine handelsübliche Zimmertür, die während der Messungen temporär abgedichtet ist. Zwei Schaufenster sind in einer Seitenwand des Modellraumes eingelassen, um z.B. Luftströmungen mit Nebel optisch verfolgen und photographisch dokumentieren zu können. Zur Beheizung des Modellraumes dient ein rollbarer Ölradiator. Das einflügelige DrehKippfenster hat die lichten Öffnungsmas̈e von 1,23 x 0,94 m (H x B).

Im Modellraum wird an zwei Stellen das Tracergas injiziert. Die Startkonzentration beträgt ca. $100 \mathrm{ppm}$. An vier Messstellen, angeordnet in unterschiedlichen Raumhöhen - 0,5 m, 1,0 m, 1,5 m und 2,0 m - wird die Raumluft entnommen und die Tracergaskonzentration vor dem Öffnen und nach dem Schließen des Fensters bestimmt (Bild 8). Die Durchmischung der Luft erfolgt vor und nach der Messung jeweils mit zwei Ventilatoren. Die Tracergas-Konzentration im Labor wird ebenfalls über vier Entnahmestellen ermittelt.

Zwei Temperaturfühler zeichnen die Lufttemperatur kontinuierlich im Labor und im Modellraum auf. Im Bereich des Fensters - 1,2 m vor dem Fenster - wird im Labor die Temperaturschichtung in Abhängigkeit von der Laborhöhe mit strahlungsabgeschirmten PT 1000-Fühlern ermittelt. Die sechs Temperaturfühler sind jeweils in einem Abstand von $1 \mathrm{~m}$ montiert. Es stellt sich zwischen dem untersten und obersten Temperaturfühler eine Temperaturdifferenz von 1 - $2 \mathrm{~K}$ ein.

Für einige Messreihen werden im Modellraum an verschiedenen Positionen die Temperaturen protokolliert. Bild 9 zeigt die Anordnung der Temperaturfühler (strahlungsabgeschirmte PT 1000). Die grau hinterlegten Kreise kennzeichnen Temperaturfühler, die die Lufttemperatur aufnehmen. Die Messstellen der Oberflächentemperaturen von Decke und Boden werden durch schwarze Kreise und die von den Wänden durch weiße Kreise markiert. Im oberen Bild ist eine 3d-Ansicht skizziert. Das mittlere Bild zeigt einen vertikalen Schnitt durch die Mitte des Raumes und das untere Bild einen horizontalen Schnitt, wobei zur Verdeutlichung die Positionen durch schwarze Kreise markiert sind, an denen die Oberflächentemperaturen 

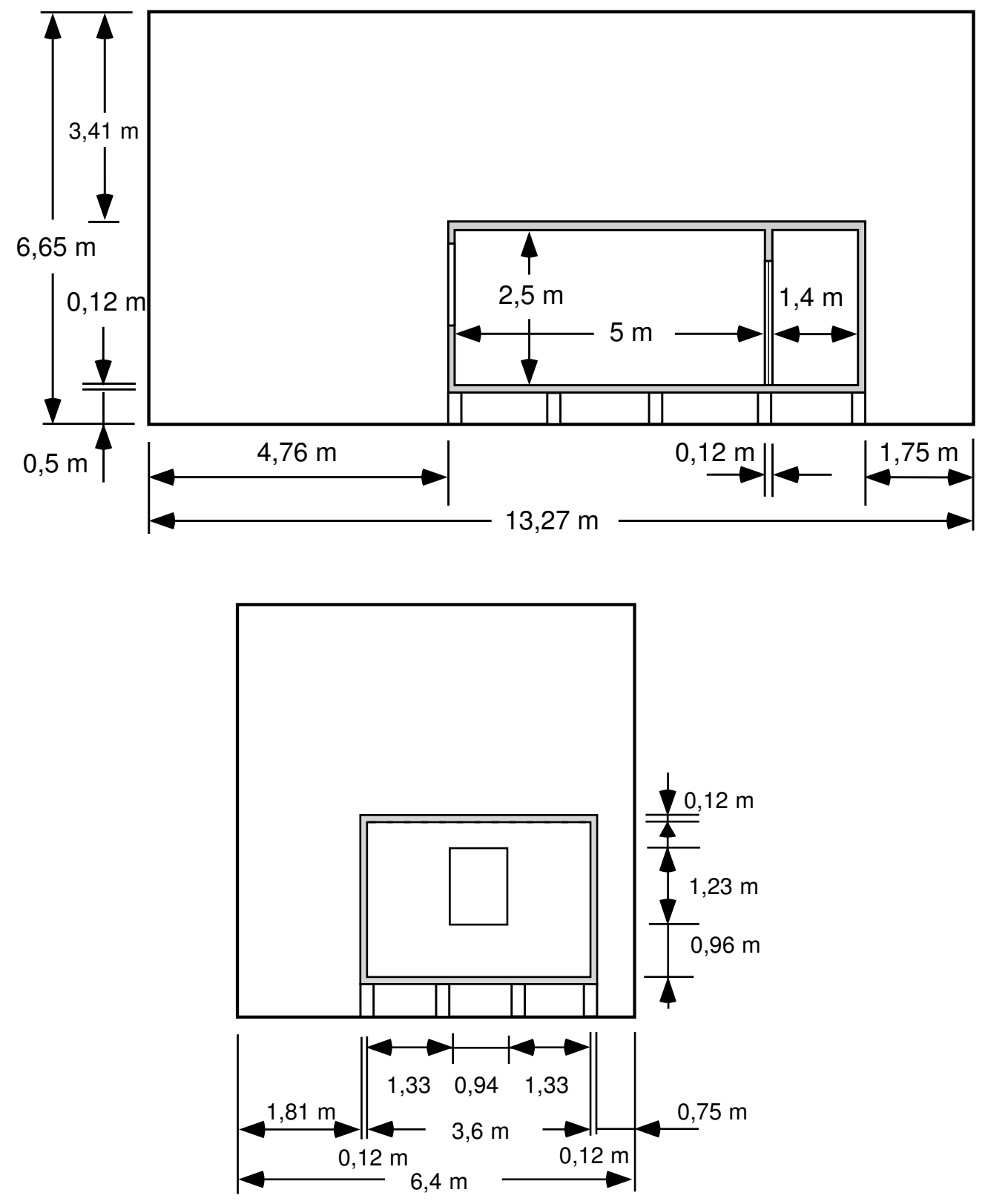

Bild 7: Skizze des Modellraums im Labor. 


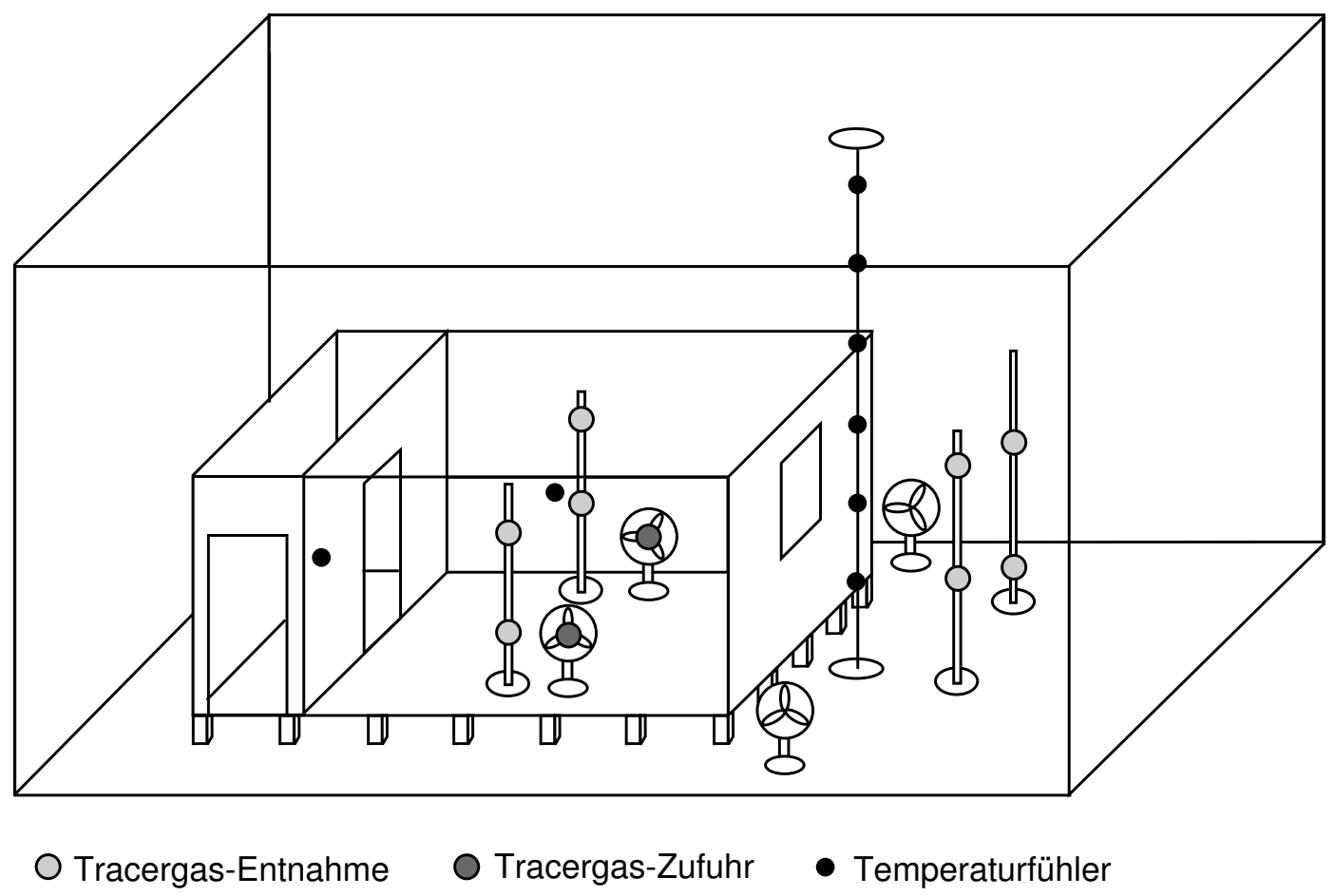

Bild 8: Anordnung der Tracergas-Zufuhr- und -Entnahmestellen, der Temperaturfühler sowie der Ventilatoren im Modellraum und im Labor.

von Boden und Decke aufgenommen werden. Die Lufttemperaturen und die Oberflächentemperaturen der Wände werden jeweils in fünf verschiedenen Höhen bestimmt. Die Temperaturen der Decken- und Bodenoberfläche werden an jeweils vier Stellen ermittelt. Mit den insgesamt 93 Temperaturfühlern soll eine detaillierte Betrachtung der Temperaturverteilung während der Lüftung mit einem Kippfenster erfolgen. Die einzelnen Temperaturfühlerketten sind mit Buchstaben gekennzeichnet und in der jeweiligen Kette werden die Temperaturfühler von unten nach oben mit einer aufsteigenden Zahl gezählt. Die Fühler der Oberflächentemperaturen von Boden und Decke sind mit dem Zusatz 'B' bzw. 'D' versehen.

Die gesamten Messungen werden zentral von einem Nachbarraum des Labors gesteuert. In diesem Raum befinden sich die Gasvorratsflaschen, der Gasanalysator sowie ein Computer zur Steuerung und Messwerterfassung. Der verwendete Infrarotabsorptions-Gasanalysator UNOR 6N der Firma Mayhak, Hamburg, dient der Messung der Tracergaskonzentrationen. Für das verwendete Messgas Distickstoffoxid $\left(\mathrm{N}_{2} \mathrm{O}\right)$ können die Bereiche 0 - 100 ppm und 100 - 500 ppm gewählt werden. Bei den im Rahmen dieser Arbeit durchgeführten Versuchen wird der Messbereich 0 - 100 ppm verwendet. Das Gerät besitzt lt. Hersteller einen maximalen Linearitätsfehler von $\leq 1 \%$ des Messbereichs-Endwertes. Die Drift des Nullpunkts und der Empfindlichkeit wird mit $\leq 1 \%$ der Messspanne pro Woche angegeben. Infolge der möglichen Messwertdrift wird an einem Messtag morgens ein Kalibrierverfahren durchgeführt und dabei der Nullpunkt mit reinem Stickstoff $\mathrm{N}_{2}$ und die Empfindlichkeitseinstellung mit einem Prüfgas bekannter $\mathrm{N}_{2} \mathrm{O}$-Konzentration 

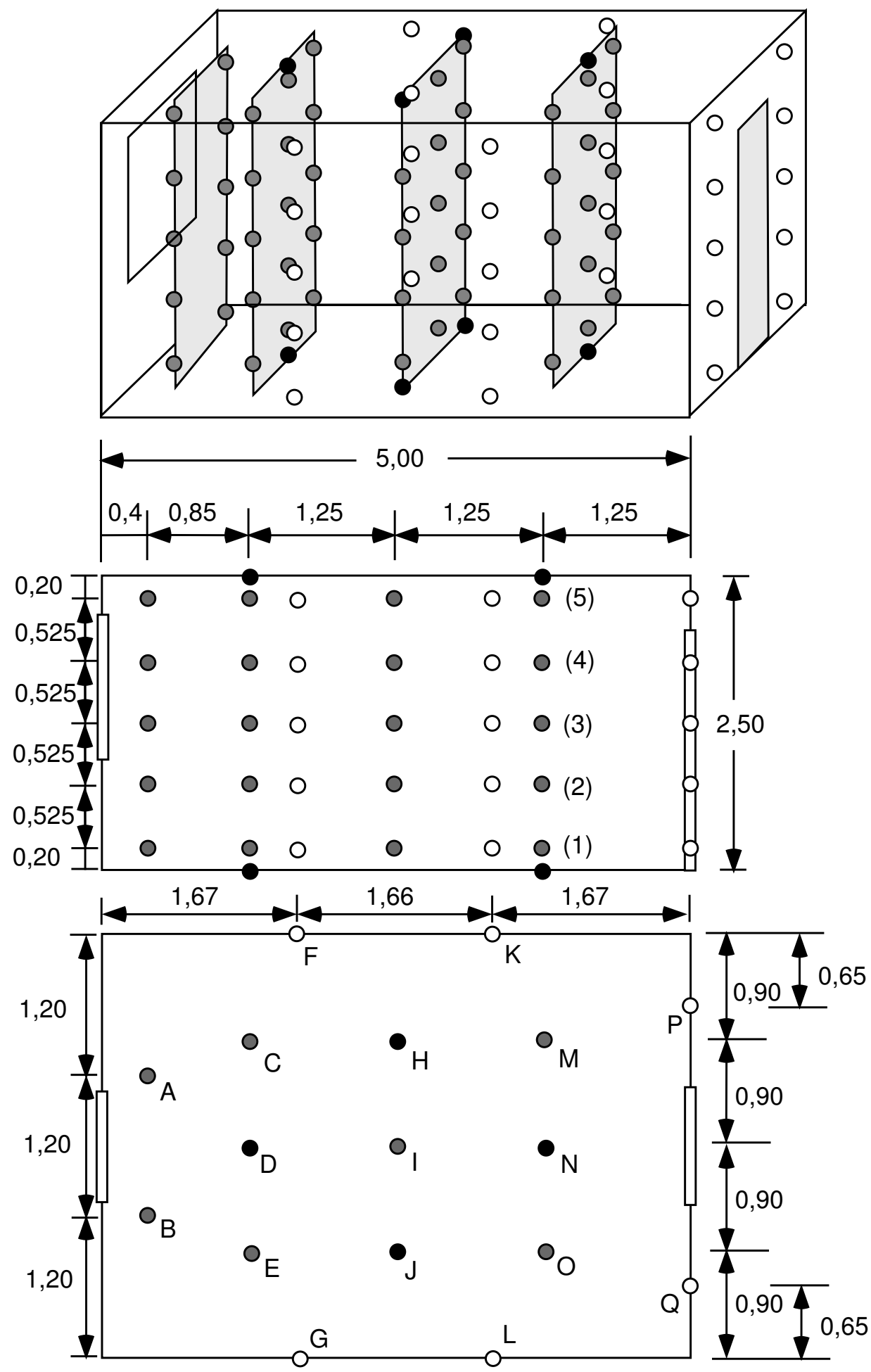

Bild 9: Anordnung der Temperaturfühler im Modellraum. Oben: 3d-Ansicht; Mitte: vertikaler Schnitt; Unten: horizontaler Schnitt. Weiße Kreise: Wandoberflächentemperaturen, graue Kreise: Lufttemperaturen, schwarze Kreise: Decken/Bodenoberflächentemperaturen. 
überprüft. Die Messdatenerfassung sowie die Steuerung der Messgaskonzentration wird mit einem Programm realisiert, welches auf LabVIEW der Firma National Instruments, München, basiert. Die einzelnen Messdaten werden alle $5 \mathrm{~s}$ aufgenommen und als 30 s Mittelwerte gespeichert. Detaillierte Angaben zu dem Messprogramm und zu den Hardwarekomponenten sowie weiteren Parametern zur Kalibration des Messsystems sind in [50, 51] zu finden.

\subsection{Ablauf der Messungen}

Der Ablauf einer Tracergasmessung nach der Konzentrations-Abfallmethode gliedert sich in mehrere Schritte:

Schritt 1 Der Modellraum wird mit einem Radiator beheizt, bis sich die gewünschte Temperaturdifferenz zwischen Modellraum und Labor einstellt. Das Fenster ist während der Aufwärmphase geschlossen. Zwei Ventilatoren unterstützen die Verteilung der warmen Luft im Modellraum.

Schritt 2 Ist die geforderte Temperaturdifferenz erreicht, wird das Tracergas in den Modellraum eingebracht und mit den beiden Ventilatoren verteilt, bis der Gasanalysator keine Änderung der Konzentration anzeigt. Die Startkonzentration im Raum beträgt für alle Versuche rund 100 ppm. Für die Untersuchungen 'ohne Heizung' wird der Radiator vor der Tracergasinjektion aus dem Raum entfernt. Das Fenster bleibt während des Einbringens des Tracergases geschlossen.

Schritt 3 Die Ventilatoren werden ausgestellt und das Fenster wird geöffnet.

Schritt 4 Ist die Raumkonzentration des Tracergases auf ca. 40 ppm abgefallen, wird das Fenster geschlossen.

Schritt 5 Die beiden Ventilatoren werden für die Durchmischung der Raumluft eingeschaltet. Zeigt der Gasanalysator eine konstante Konzentration an, wird die Messung beendet.

Aus den Anfangs- und Endkonzentrationen und den Temperaturen im Modellraum und Labor sowie der Fensteröffnungszeit, wird der Zuluftvolumenstrom nach Gleichung 3 bestimmt. 


\subsection{Messunsicherheit}

Der gesuchte Volumenstrom ist mit der Tracergas-Technik nicht direkt zu ermitteln. Er wird aus mehreren Einzelgrößen berechnet, die ihrerseits jedoch unmittelbar gemessen werden können. Um die Gesamtunsicherheit des bestimmten Volumenstroms abzuschätzen, werden zuerst die Unsicherheiten der direkt gemessenen Größen ermittelt, und anschließend mit Hilfe der Einzelunsicherheiten die Gesamtunsicherheit bestimmt. Hierbei wird angenommen, dass die einzelnen Messgrößen von einander unabhängig und zufällig sind.

In allgemeiner Form lässt sich die Unsicherheit $\delta \mathrm{q}$ einer Funktion q(i) mit mehreren Variablen $\mathrm{i}=1 \ldots \mathrm{k}$ und deren Unsicherheiten $\delta \mathrm{x}_{1}, \ldots, \delta \mathrm{x}_{\mathrm{k}}$ durch

$$
\delta q=\sqrt{\sum\left(\delta x_{i} \frac{\partial q}{\partial x_{i}}\right)^{2}}
$$

abbilden. Die Unsicherheiten $\delta \mathrm{x}_{\mathrm{i}}$ sind voneinander unabhängig und zufällig [55].

Die Unsicherheit, die bei der Bestimmung des Volumenstroms mit der Konzentrationsabfall-Methode entsteht, setzt sich aus den Ungenauigkeiten der Bestimmung der Raum- und Umgebungslufttemperatur, der Versuchsdauer und der Bestimmung der Anfangs- und Endkonzentration des Tracergases zusammen.

Die Lufttemperatur wird mit einem Fühler der Firma Vaisala Oy, Helsinki, bestimmt. Lt. Herstellerangabe weist die Temperaturbestimmung eine Unsicherheit von $\pm 0,2 \mathrm{~K}$ bei $20^{\circ} \mathrm{Cauf}$. Mit der Annahme, dass die Luft nicht ganz vollständig durchmischt ist, wird die Genauigkeit der Temperaturbestimmung zu 0,5 K gesetzt.

Die Bestimmung der Versuchsdauer erfolgt über einen Fensterkontakt. Der Fehler der Zeitbestimmung beträgt $\pm 1 \mathrm{~s}$.

Die Ungenauigkeit der $\mathrm{N}_{2} \mathrm{O}$-Konzentrationsmessung ergibt sich aus folgenden Anteilen:

- Der Linearitätsfehler des Analysators beträgt bis zu $1 \%$ der Messspanne. Bei dem verwendeten Messbereich von 0 - 100 ppm ergibt sich ein Fehler von maximal $1 \mathrm{ppm}$.

- Die Kalibration des Messgerätes mit zwei verschiedenen Prüfgasen per Hand kann zu einer Ungenauigkeit führen.

- Unvollständige Durchmischung des Tracergases.

- Die Nullpunkt- und Empfindlichkeitsdrift des Gasanalysators wird lt. Hersteller mit $\leq 1 \%$ des Messbereich-Endwertes pro Woche angegeben. Da nur Kurzzeitmessungen durchgeführt werden, kann die Drift vernachlässigt werden. 
Für eine ideale Durchmischung und Einstellung des Gerätes ist mit einer Ungenauigkeit der Konzentrationsbestimmung von maximal 1 ppm zu rechnen. Infolge unvollständiger Durchmischung und Unsicherheit in der Kalibration des Messgerätes wird eine maximale Ungenauigkeit von $3 \mathrm{ppm}$ angenommen.

Eine genaue Quantifizierung der Ungenauigkeit bei der Bestimmung der TracergasKonzentration ist für die durchgeführten Messreihen nicht möglich. Die Ermittlung des Temperaturfeldes während der Messungen zeigt jedoch, dass bei den Varianten 'ohne Heizung' während der Mischdauer alle Lufttemperaturfühler annähernd gleiche Temperaturen anzeigen (Abschnitt TempImModellraum1). Es wird davon ausgegangen, dass infolge des homogenen Temperaturfeldes auch eine homogene Konzentrationsverteilung vorliegt. Für die Varianten 'mit Heizung' liegt während der Mischdauer zwar eine Temperaturschichtung vor (Abschnitt TempImModellraum2), jedoch kann auch hierbei eine vollständige Durchmischung angenommen werden. Die durch die Heizung induzierte Luftbewegung reicht nach [16] aus, um eine homogene Vermischung sicherzustellen.

Über die Modellraumhülle wird ein $\mathrm{n}_{50}$-Wert $\approx 0,06 \pm 0,02 \mathrm{~h}^{-1}$ bestimmt [23], d.h. der Modellraum ist im Rahmen der Tracergasmessung als dicht zu erachten.

Die Betrachtung der einzelnen Einflussgrößen zeigt, dass der Gesamtfehler der Volumenstrombestimmung hauptsächlich von der Unsicherheit der Konzentrationsbestimmung des Tracergases abhängig ist. Die Gesamtunsicherheit der ermittelten Volumenströme liegt mit o.g. Annahmen in dem Bereich zwischen $\pm 3 \%$ für die 'idealen Bedingungen' und $\pm 10 \%$ infolge der 'konservativen' Schätzung der Ungenauigkeit der Konzentrationsbestimmung. 


\section{Messergebnisse}

\subsection{Tracergas-Messungen}

\subsubsection{Darstellung der Zuluftvolumenströme}

Der Zuluftvolumenstrom wird für die Standard-Kippweite des Fensters von $10 \mathrm{~cm}$ und die beiden reduzierten Kippweiten von $3 \mathrm{~cm}$ und $6 \mathrm{~cm}$ unter verschiedenen Randbedingungen mit der Konzentrationsabfall-Methode bestimmt. Die Anordnung der Laibung (L) - nur auf der Innen- / Außenseite bzw. auf beiden Seiten des Fensters - und der Heizung $(\mathrm{H})$ - Heizkörper unter dem Fenster resp. ohne Heizkörper - variiert. Bei den Varianten 'ohne Heizung' wird der Heizkörper nach dem Erreichen der Soll-Raumtemperatur aus dem Versuchsraum entfernt, so dass während der Messung der Luftwechsel nur infolge der Temperaturdifferenz zwischen innen und außen stattfindet.

Die prozentualen Angaben zur Reduktion des Volumenstroms beziehen sich, wenn nicht anders angegeben, auf die Variante 'ohne Laibung / ohne Heizung' und einer Temperaturdifferenz zwischen innen und außen von $20 \mathrm{~K}$. Andere Werte können dem Anhang (Bild fig:Prozvonoo) entnommen werden. Grundsätzlich ist, wenn von einer Temperaturdifferenz gesprochen wird, immer die Temperaturdifferenz zwichen innen und außen gemeint.

In Bild 10 sind die ermittelten Zuluftvolumenströme in Abhängigkeit von der Temperaturdifferenz für die Kippweite von $10 \mathrm{~cm}$ aufgetragen. Im oberen Teil sind die Varianten 'ohne Heizung', im unteren Teil die Varianten 'mit Heizung' zusammengestellt. Die einzelnen Randbedingungen der dargestellten Ergebnisse lauten

Variante 1 Das Fenster wird ohne Laibung und ohne Heizung untersucht. Der Fensterrahmen schließt auf der Warm- und Kaltseite nahezu bündig mit der Außenwand ab. Der Heizkörper ist während der Messdauer entfernt (schwarze Kreise, Variante 'ohne Laibung / ohne Heizung').

Variante 2 Eine raumseitige Laibung von $20 \mathrm{~cm}$ Tiefe wird angebracht. Der Abstand der Laibung zum seitlichen Fensterblendrahmen beträgt $4,3 \mathrm{~cm}$, im Bereich des Sturzes 5,5 cm (schwarzes Quadrat, Variante 'mit Laibung / ohne Heizung').

Variante 3 Eine Laibung von $20 \mathrm{~cm}$ Tiefe befindet sich auf der Außenseite des Fensters. Diese Variante wird ohne Heizung durchgeführt (weißes Quadrat).

Variante 4 Untersucht wird die gleichzeitige Anordnung einer Laibung auf der Innen- und Außenseite von jeweils $20 \mathrm{~cm}$ Laibungstiefe (ohne Heizung, weiße Dreiecke).

Variante 5 Das Kippfenster wird ohne Laibung jedoch mit Heizung untersucht. Der Heizkörper ist während der Messung mittig, unterhalb des Fensters angeordnet und ist über die gesamte Messdauer eingeschaltet (weiße Kreise, Variante 'ohne Laibung / mit Heizung'). 
Variante 6 Das Kippfenster wird mit einer $20 \mathrm{~cm}$ tiefen Laibung auf der Innenseite und einem unterhalb des Fensters angeordneten Heizkörpers untersucht. Der Heizkörper ist über die gesamte Messdauer eingeschaltet (weiße Quadrate, Variante 'mit Laibung / mit Heizung').

Der Vergleich der Messergebnisse zeigt, dass die Anordnung der Laibung und eines Heizkörpers entscheidende Einflüsse auf den Zuluftvolumenstrom haben. Während eine auf der Außenseite des Fensters angeordnete Laibung von $20 \mathrm{~cm}$ keine Auswirkungen auf den Volumenstrom hat, reduziert sich infolge einer raumseitig angeordneten Laibung von $20 \mathrm{~cm}$ der Volumenstrom um rund $15 \%$ gegenüber der Variante 'ohne Laibung / ohne Heizung'. Für ein Fenster ohne Laibung, jedoch mit einem unterhalb des Fensters angeordneten Heizkörper ist eine Reduktion von ca. $22 \%$ zu verzeichnen. Die gleichzeitige Anordnung einer raumseitigen Laibung und eines Heizkörpers unterhalb des Fensters bewirkt eine Abnahme des Zuluftvolumenstroms um ca. 42 \% gegenüber der Variante 'ohne Laibung / ohne Heizung'.

Analoge Versuche von [37] weisen für eine Kippweite von $10 \mathrm{~cm}$ eine Reduktion des Zuluftvolumenstroms von ca. $37 \%$ auf, bei einer Laibungstiefe von $20 \mathrm{~cm}$ und einem Laibungsabstand von $2 \mathrm{~cm}$. D.h. bei einer Kombination von Laibung und Heizung muss mit einer Reduktion des Luftwechselpotentials bei Kippfenstern mit Standard-Kippweite von ca. $40 \%$ gegenüber der Variante 'ohne Laibung / ohne Heizung' gerechnet werden.

In Bild 11 sind exemplarisch Volumenströme von vier Varianten über der Wurzel der Temperaturdifferenz - in Anlehnung an die Literaturansätze - aufgetragen. Im Idealfall sollten die extrapolierten Kurven durch den Nullpunkt laufen, da ohne Temperaturdifferenz außer dem Konzentrationsunterschied keine treibende Kraft anliegt. Die Extrapolationen zeigen, dass dies nur bei drei Varianten annähernd der Fall ist. Aus dem Bild ist abzuleiten, dass für die Variante 'mit Laibung / ohne Heizung' eine Mindesttemperaturdifferenz vorliegen muss, damit ein Luftwechsel initiiert wird. Es ist anzunehmen, dass dieses Verhalten auf die Verengung des Strömungsquerschnittes und den veränderten Strömungsverhältnissen infolge der Laibung zurückzuführen ist.

Der Einfluss der unterschiedlichen Laibungstiefe bzw. -anordnungen ist in Bild 12 dargestellt. Die verschiedenen Laibungstiefen sollen unterschiedliche Einbausituationen des Fensters widerspiegeln. Für die Kippweite von $10 \mathrm{~cm}$ werden die Laibungstiefen $10 \mathrm{~cm}$ und $20 \mathrm{~cm}$, jeweils mit einem Laibungsabstand von $4,3 \mathrm{~cm}$, untersucht. Die Laibung von $10 \mathrm{~cm}$ Tiefe entspricht der Situation, dass die Laibung oberhalb des Kippflügels endet. Bei einer Laibungstiefe von $20 \mathrm{~cm}$ ragt die Laibung um mehrere Zentimeter über den Fensterflügel hinweg in den Raum. Die Messungen erfolgen ohne Heizung.

Wie bereits dargestellt wurde, hat eine auf der Außenseite des Fensters angeordnete Laibung der Tiefe $10 \mathrm{~cm}$ bzw. $20 \mathrm{~cm}$ keinen messbaren Einfluss auf das Lüftungsverhalten. Die Messergebnisse für die beiden unterschiedlichen raumseitigen Laibungstiefen zeigen, dass eine Laibung auf der Innenseite des Fensters den Volumenstrom beeinflusst. Ist die Laibungstiefe mindestens genauso groß wie die Kippweite, 

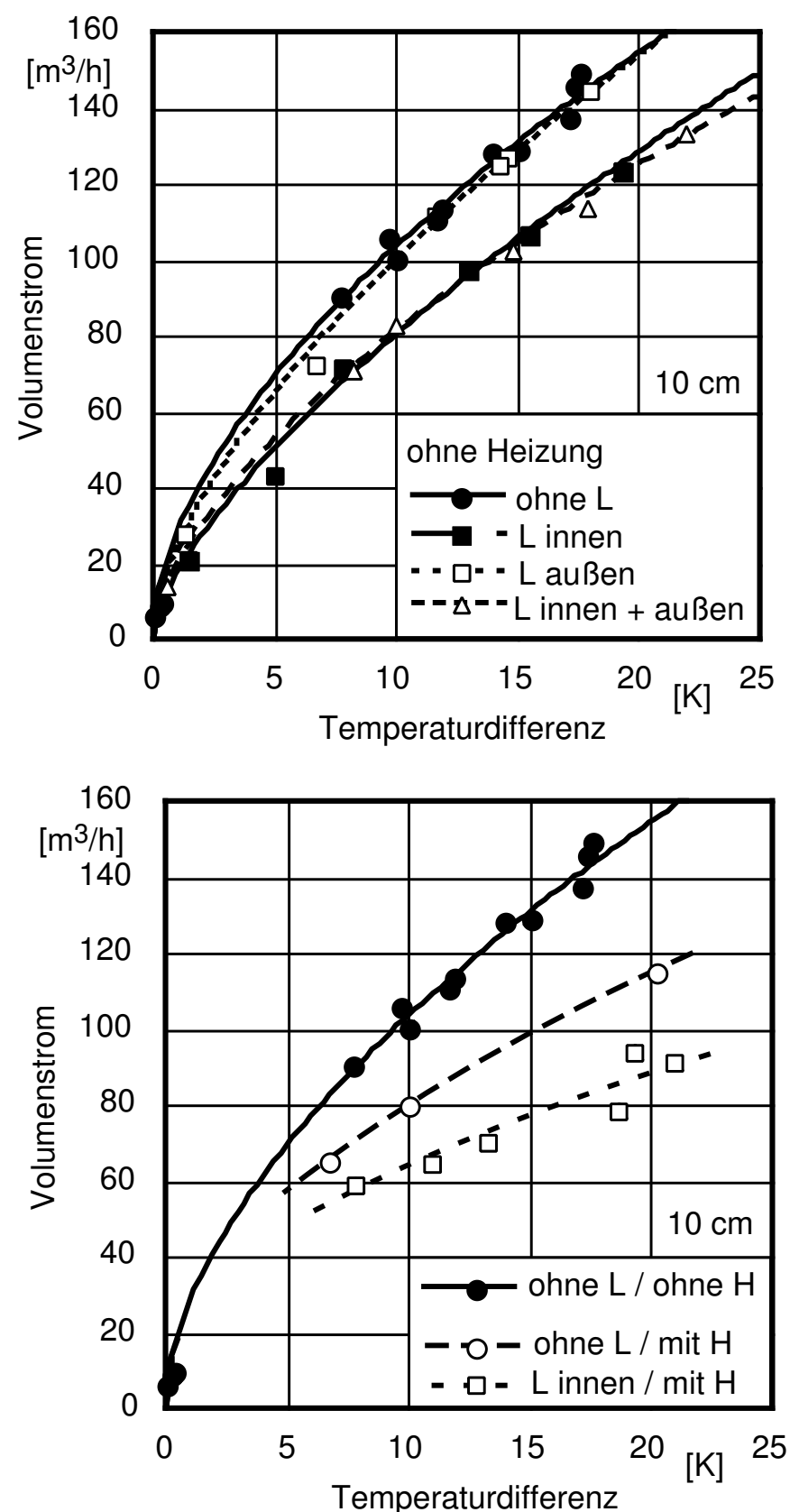

Bild 10: Zuluftvolumenstrom in Abhängigkeit von der Temperaturdifferenz für die Standard-Kippweite von $10 \mathrm{~cm}$ (L: Laibung, H: Heizung). 


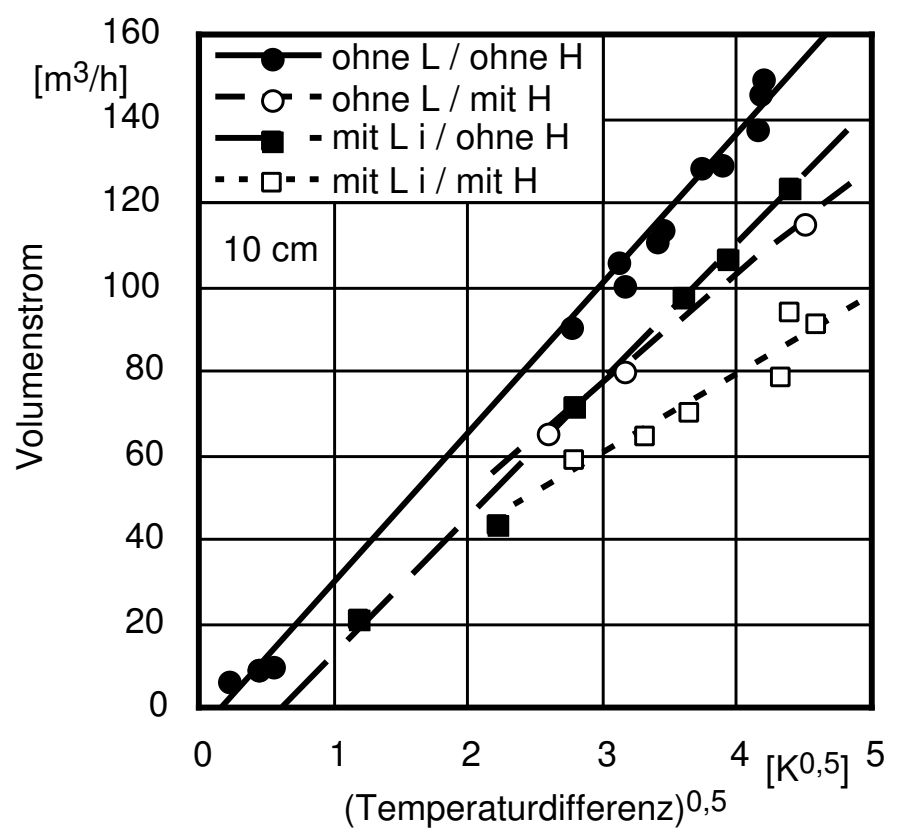

Bild 11: Zuluftvolumenstrom in Abhängigkeit von der Wurzel der Temperaturdifferenz für die Standard-Kippweite von $10 \mathrm{~cm}(20 \mathrm{~cm}$ Laibung innen).

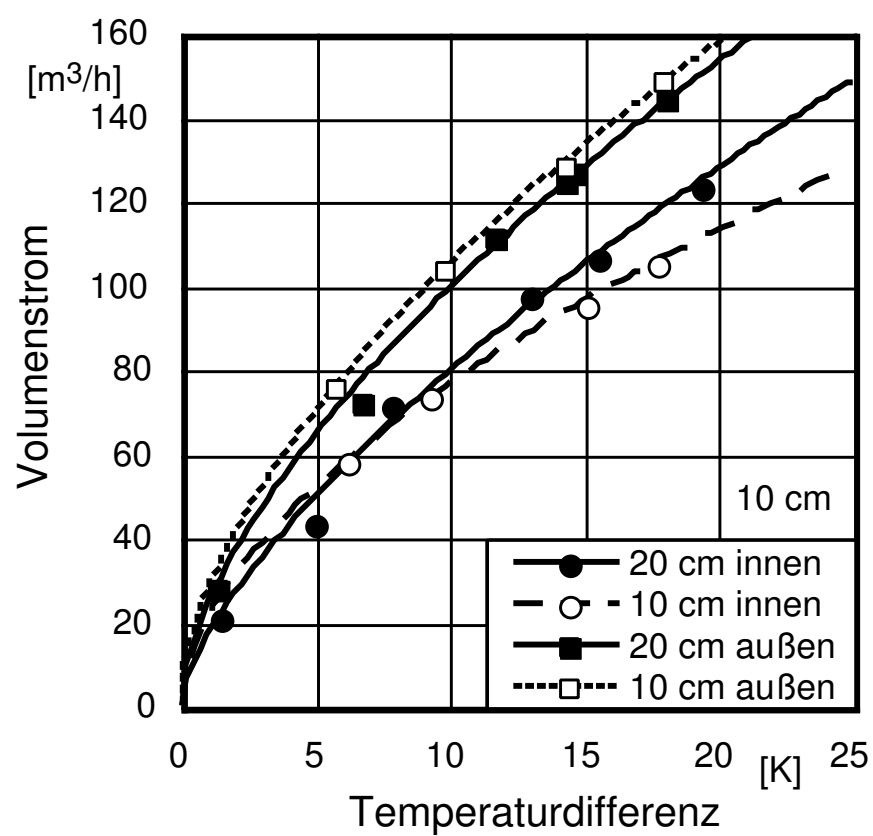

Bild 12: Zuluftvolumenstrom in Abhängigkeit von der Temperaturdifferenz für die Kippweite von $10 \mathrm{~cm}$ für unterschiedliche Laibungstiefen und -anordnungen (ohne Heizung). 
spielt die Tiefe der Laibung eine untergeordnete Rolle. Erst ab einer Temperaturdifferenz größer $20 \mathrm{~K}$ weicht der Zuluftvolumenstrom bei einer innenliegende Laibung von $10 \mathrm{~cm}$ Tiefe mehr als $10 \%$ von dem Volumenstrom bei einer Laibungstiefe von $20 \mathrm{~cm} \mathrm{ab.}$ 
Die Bilder 13 und 14 zeigen die gemessenen Zuluftvolumenströme in Abhängigkeit von der Temperaturdifferenz für die reduzierten Kippweiten von $3 \mathrm{~cm}$ und $6 \mathrm{~cm}$. Es werden die Varianten

- ohne Laibung / ohne Heizung,

- ohne Laibung / mit Heizung,

- mit $20 \mathrm{~cm}$ Laibung (innen) / ohne Heizung und

- mit $20 \mathrm{~cm}$ Laibung (innen) / mit Heizung

untersucht.

Für die Kippweite von $6 \mathrm{~cm}$ kann infolge der raumseitigen Laibung bei den Variante 'ohne Heizung' nur bei kleineren Temperaturdifferenzen - bis ca. $15 \mathrm{~K}$, eine reduzierende Wirkung festgestellt werden (Bild 13 oben). Die Variante 'ohne Laibung / mit Heizung' führt zu einem verminderten Volumenstrom von ca. $17 \%$. Die Kombination aus Laibung und Heizung ergibt eine Reduktion von rund $25 \%$.

Im unteren Teil von Bild 13 ist ebenfalls der Volumenstrom über der Wurzel der Temperaturdifferenz aufgetragen. Würde man die Kurven für die Varianten 'mit Heizung' extrapolieren, könnte man für diese Varianten einen Volumenstrom bei einer Temperaturdifferenz von $0 \mathrm{~K}$ bestimmen. Diese Aussage ist als nicht korrekt anzusehen, da bei einer Temperaturdifferenz von $0 \mathrm{~K}$ keine Heizung vorhanden ist und damit kein Luftaustausch stattfindet. Die Tendenz der Kurven zeigt jedoch, dass bei niedrigen Temperaturdifferenzen der Luftwechsel durch das Vorhandensein einer Heizung verstärkt werden würde. 

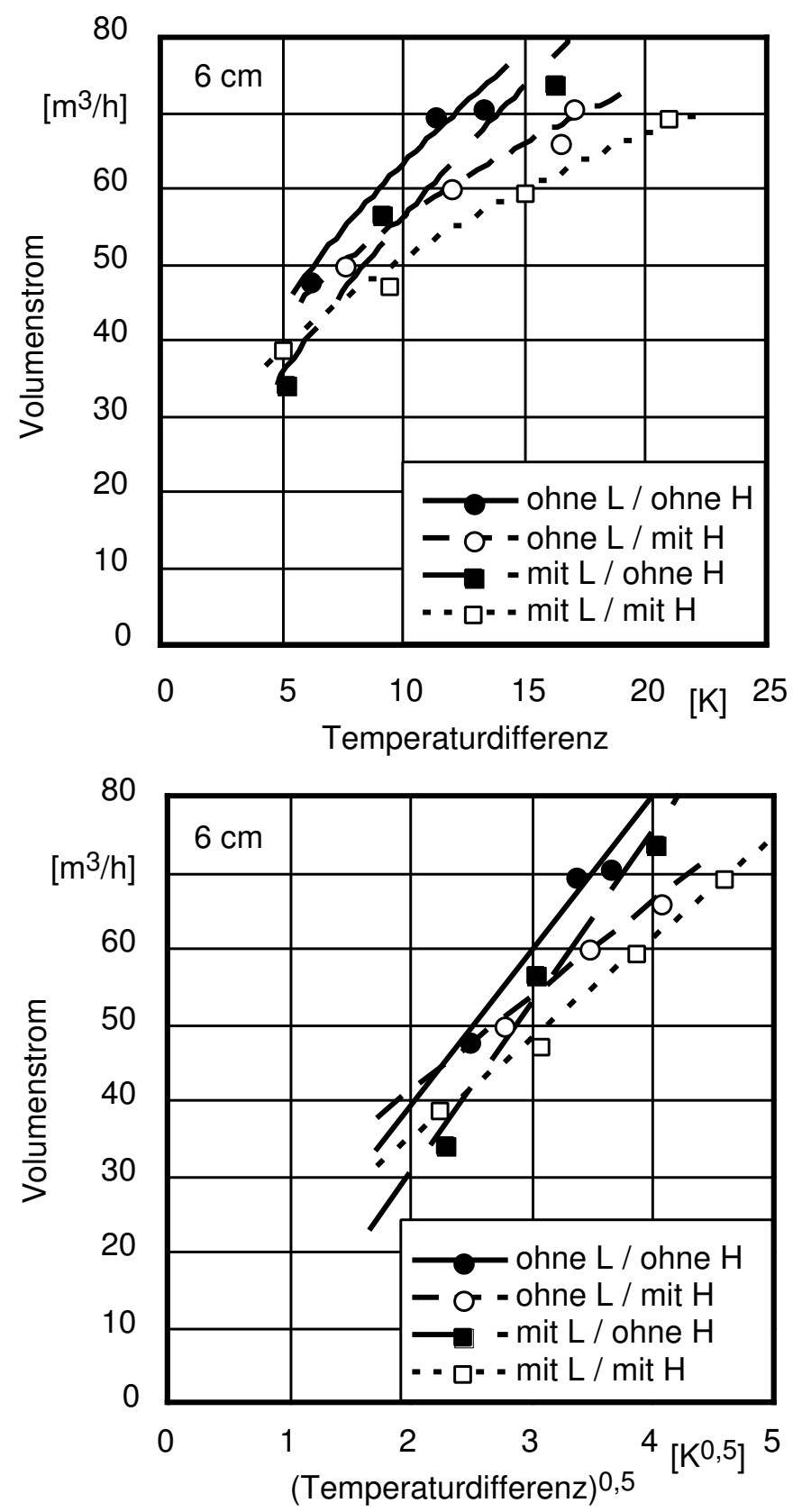

Bild 13: Zuluftvolumenstrom in Abhängigkeit von der Temperaturdifferenz für die reduzierte Kippweite von $6 \mathrm{~cm}$.. 

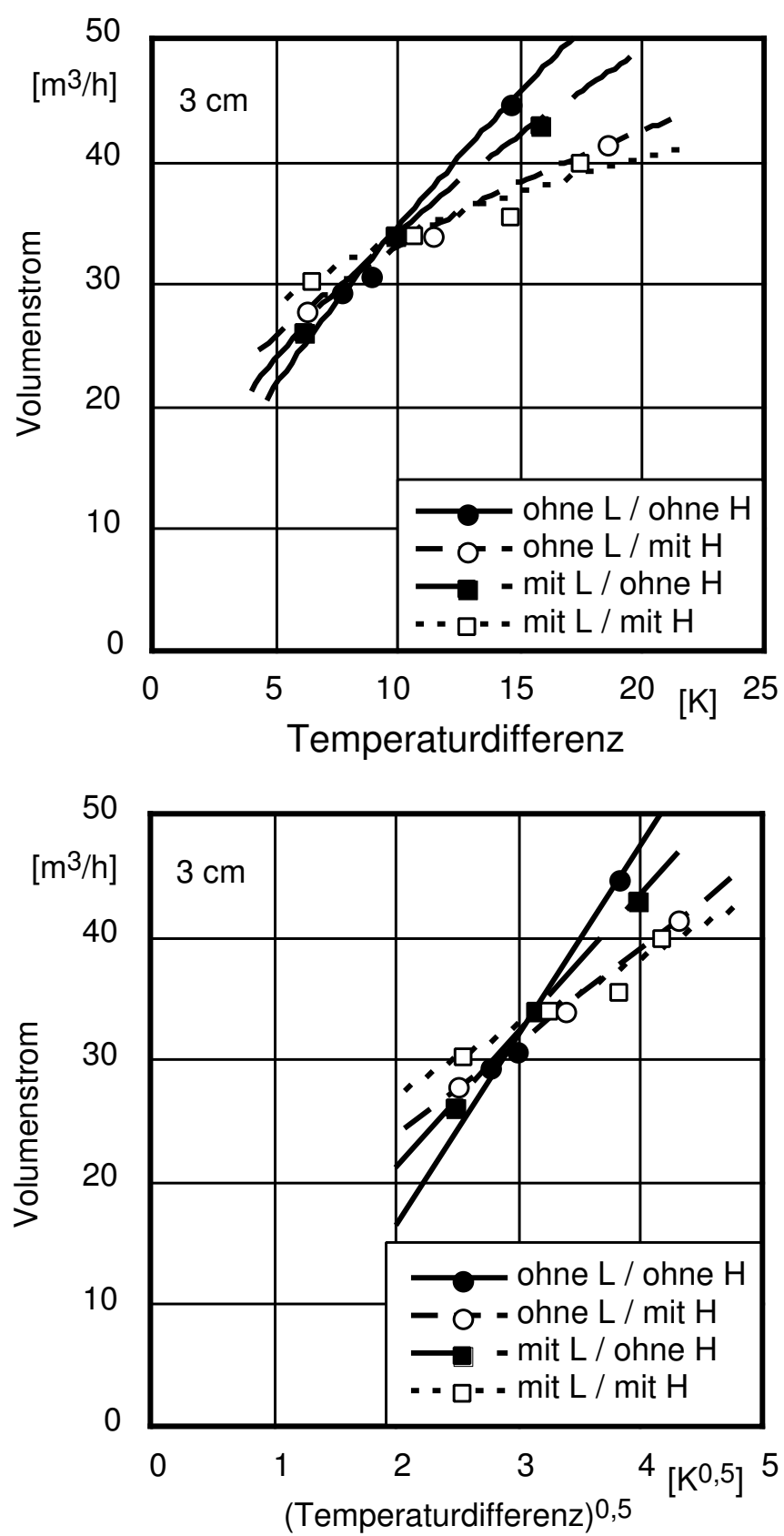

Bild 14: Zuluftvolumenstrom in Abhängigkeit von der Temperaturdifferenz für die reduzierte Kippweite von $3 \mathrm{~cm}$.

Die Messergebnissse für die reduzierte Kippweite von $3 \mathrm{~cm}$ sind in Bild 14 dargestellt. Für Temperaturdifferenzen größer $10 \mathrm{~K}$ entsprechen die Tendenzen der Messergebnisse den Kippweiten $6 \mathrm{~cm}$ und $10 \mathrm{~cm}$. Die Variante 'ohne Laibung / ohne Heizung' führt zu den größten, die Variante 'mit Laibung / mit Heizung' zu den geringsten Volumenströmen. Bei einer Temperaturdifferenz kleiner als $10 \mathrm{~K}$ wird durch die Extrapolation darauf hingedeutet, dass dieses Verhalten genau umgekehrt ist, d.h. durch einen unterhalb des Fensters angeordneten Heizkörpers wird der Luftaustausch bei niedrigen Temperaturdifferenzen verstärkt. Die raumseitige Laibung führt zu einer Reduktion von knapp $10 \%$. Die Varianten 'mit Heizung' 
weisen einen um ca. $25 \%$ geringeren Volumenstrom auf.

\subsubsection{Vergleich unterschiedlicher Kippweiten}

Der Einfluss einer innenliegenden Laibung von $20 \mathrm{~cm}$ Tiefe ist insbesondere bei der Standard-Kippweite von $10 \mathrm{~cm}$ zu verzeichnen. Die Reduktion zu der Variante 'ohne Laibung' beträgt ca. $15 \%$.

Für alle drei Kippweiten wird der Volumenstrom durch das Vorhandensein einer Heizung - Anordnung unterhalb des Fensters - verringert. Während bei einer Kippweite von $3 \mathrm{~cm}$ und $10 \mathrm{~cm}$ eine Reduktion von $22 \%$ stattfindet, beträgt sie bei den Kippweiten von $6 \mathrm{~cm}$ ca. $17 \%$.

Eine Kombination von raumseitiger Laibung und Heizung vermindert das Luftwechselpotential um rund $42 \%$ bei der Standardkippweite und um ca. $25 \%$ bei den reduzierten Kippweiten.

Signifikante Unterschiede der Volumenströme infolge der unterschiedlichen Randbedingungen treten bei $10 \mathrm{~cm}$ Kippweite ab einer Temperaturdifferenz von rund 5 $\mathrm{K}$, bei den anderen beiden Kippweiten ab einer Differenz größer ca. $10 \mathrm{~K}$ auf.

Bei den reduzierten Kippweiten weist die Extrapolation darauf hin, dass der Luftwechsel durch das Vorhandensein einer Heizung bei kleinen Temperaturdifferenzen verstärkt wird.

Wie erwartet, zeigt der Vergleich der Zuluftvolumenströme über die drei untersuchten Kippweiten, dass für die Variante 'ohne Laibung / ohne Heizung' der Volumenstrom für eine bestimmte Temperaturdifferenz annähernd linear mit der Öffnungsfläche zu- bzw. abnimmt. Bei der Temperaturdifferenz von $15 \mathrm{~K}$ beträgt für die Kippweite $10 \mathrm{~cm}$ der Volumenstrom ca. $130 \mathrm{~m}^{3} / \mathrm{h}$ (100\%), für die Kippweite von 6 cm ca. $78 \mathrm{~m}^{3} / \mathrm{h}(\approx 60 \%)$ und für die Kippweite von $3 \mathrm{~cm} \mathrm{ca.} 46 \mathrm{~m}^{3} / \mathrm{h}(\approx 35 \%)$.

Die Variante 'mit Laibung / mit Heizung' und einer Temperaturdifferenz von 15 $\mathrm{K}$ weist diese lineare Abhängigkeit nicht mehr auf. Der Volumenstrom über die Kippweite von $6 \mathrm{~cm}$ erreicht rund $77 \%$ und über die Kippweite von $3 \mathrm{~cm}$ knapp 49 $\%$ des maximalen Volumenstroms über die Kippweite von $10 \mathrm{~cm}$ (100\%).

Bei der Messreihe 'Kippweite $6 \mathrm{~cm}, \Delta \mathrm{T}=17 \mathrm{~K}$, ohne Laibung / mit Heizung', werden die Oberflächentemperaturen des Heizkörpers ermittelt. Sie betragen bis zu $100^{\circ} \mathrm{Cbei}$ ca. $1200 \mathrm{~W}$ während der Messung. Diese hohen Temperaturen sind notwendig, um gegenüber dem nichttemperierten Labor die gewünschten Temperaturdifferenzen zwischen Modellraum und Labor aufrecht zu erhalten. In der Realität sind solch hohe Temperaturen nicht anzutreffen. Dass diese Übertemperatur für die durchgeführten Untersuchungen akzeptabel ist, wird in folgender Überlegung deutlich.

Gängige Systemtemperaturen für den Vor- und Rücklauf von Heizungen betragen $70^{\circ} \mathrm{C} / 55^{\circ} \mathrm{C}[8]$. Es wird angenommen, dass der Heizkörper eine mittlere Temperatur von $63^{\circ} \mathrm{Caufweist}$. Bei einer Raumlufttemperatur von $20^{\circ} \mathrm{Cergibt}$ sich eine Temperaturdifferenz zwischen Heizkörper und Raumluft von $43 \mathrm{~K}$. Im Experiment beträgt 
die Raumlufttemperatur $40^{\circ} \mathrm{Cund}$ damit liegt eine Temperaturdifferenz von $60 \mathrm{~K}$ an. Für die Strömungsgeschwindigkeit w bei freier Konvektion gilt der Zusammenhang: $w \sim \sqrt{\Delta T}$ [41]. Mit den o.g. Temperaturdifferenzen ergibt sich, dass die Strömungsgeschwindigkeit in der Realität rund $15 \%$ kleiner als im Experiment ist. Die Übertemperatur führt infolge der etwas erhöhten Strömungsgeschwindigkeit dazu, dass der Einfluss der Heizung leicht überbewertet wird, in der Tendenz aber richtig ist. Ein Vergleich mit Literaturwerten ist nicht möglich, da weder in [37] noch in [16] die Oberflächentemperaturen der eingesetzten Heizkörper angegeben sind.

\subsubsection{Einfluss von Heizung, Ventilatoren und Gardine}

Die Anordnung der Heizung im Modellraum wird bei einer Kippweite von $10 \mathrm{~cm}$ ohne Laibung variiert (Bild 15). Zum einen ist der Heizkörper mittig unter dem Fenster und zum anderen mittig an der dem Fenster gegenüberliegenden Wand positioniert. Für die Variante 'Wand' wird im Schnitt ein um ca. $20 \%$ höherer Volumenstrom ermittelt als für Variante 'Fenster'. Die Tendenz des Ergebnisses stimmt mit den Strömungsbetrachtungen von [49, 48] und [39] überein. Für die Planung ist also die Angabe, wo die Heizung im Verhältnis zum Fenster positioniert ist, wichtig.

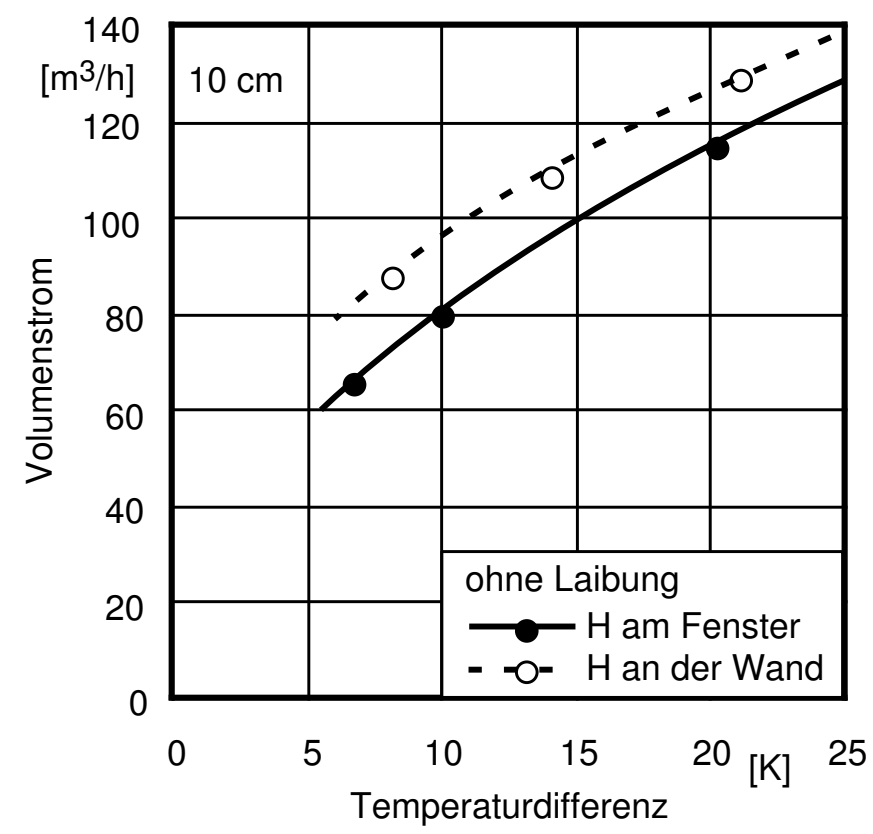

Bild 15: Zuluftvolumenstrom unter Berücksichtigung der Heizungsanordnung im Raum (Kippweite $10 \mathrm{~cm}$ ).

In Bild 16 sind Messergebnisse für die Variante 'ohne Laibung / ohne Heizung' und 'mit / ohne Ventilatoren' dargestellt. Der Einfluss von vier Tischventilatoren, die während der Messung die Luft verwirbeln um eine bessere Durchmischung der Luft zu gewährleisten, wird untersucht. Es ist deutlich zu erkennen, dass im Bereich sehr niedriger Temperaturdifferenzen die Ventilatoren zu größeren und bei 
höheren Temperaturdifferenzen zu geringeren Volumenströmen führen. Die Reduktion bei $15 \mathrm{~K}$ Temperaturdifferenz beträgt knapp 40 \%. Aus den Ergebnissen ist zu schließen, dass bei Untersuchungen von rein thermisch induzierten Luftwechseln der Einsatz von Ventilatoren zur Unterstützung der Durchmischung die Resultate deutlich beeinflusst.

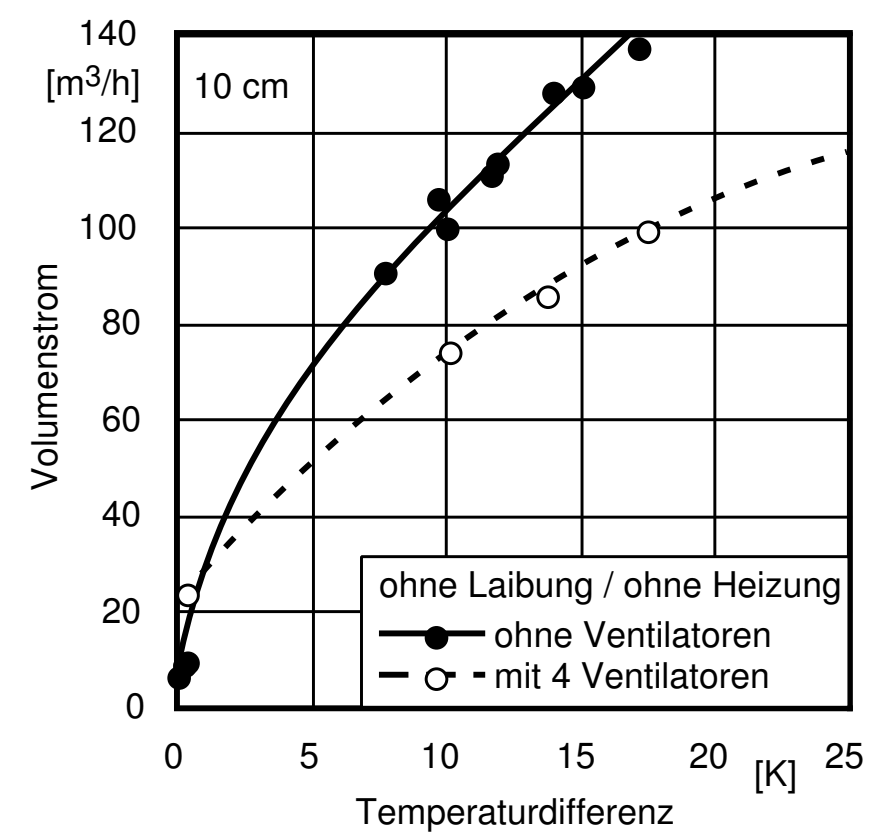

Bild 16: Zuluftvolumenstrom unter Berücksichtigung von vier Tischventilatoren (Kippweite $10 \mathrm{~cm}$ ).

Die Untersuchungen mit den unterschiedlichen Anordnungen der Heizung im Raum bzw. mit den Ventilatoren zeigen, dass der Luftwechsel durch der Raumausstattung bzw. -nutzung beeinflusst wird. Bei der Auslegung von Räumen in denen z.B. Maschinen plaziert werden sollen, die eine erhöhte Wärmeabgabe oder Ventilatoren zur Kühlung aufweisen, ist mit einem anderen Luftwechsel zu rechnen als bei einem leeren Raum.

Der Einfluss einer Gardine wird bei der Variante 'mit Laibung / ohne Heizung' untersucht. Es kann eine Reduktion des Volumenstroms um ca. $10 \%$ für Temperaturdifferenzen größer als $20 \mathrm{~K}$ festgestellt werden (Bild 17), d.h. bei geringen Temperaturdifferenzen stellt die untersuchte Gardine keinen Strömungswiderstand dar. Dieses Ergebnis ist jedoch nicht auf weniger durchlässige Stoffe zu übertragen. In [16] wird für durchlässige Stores als Orientierung eine Abminderung von 6 - 10 $\%$ und 15 - $30 \%$ für einen undurchlässigen Vorhang angegeben. 


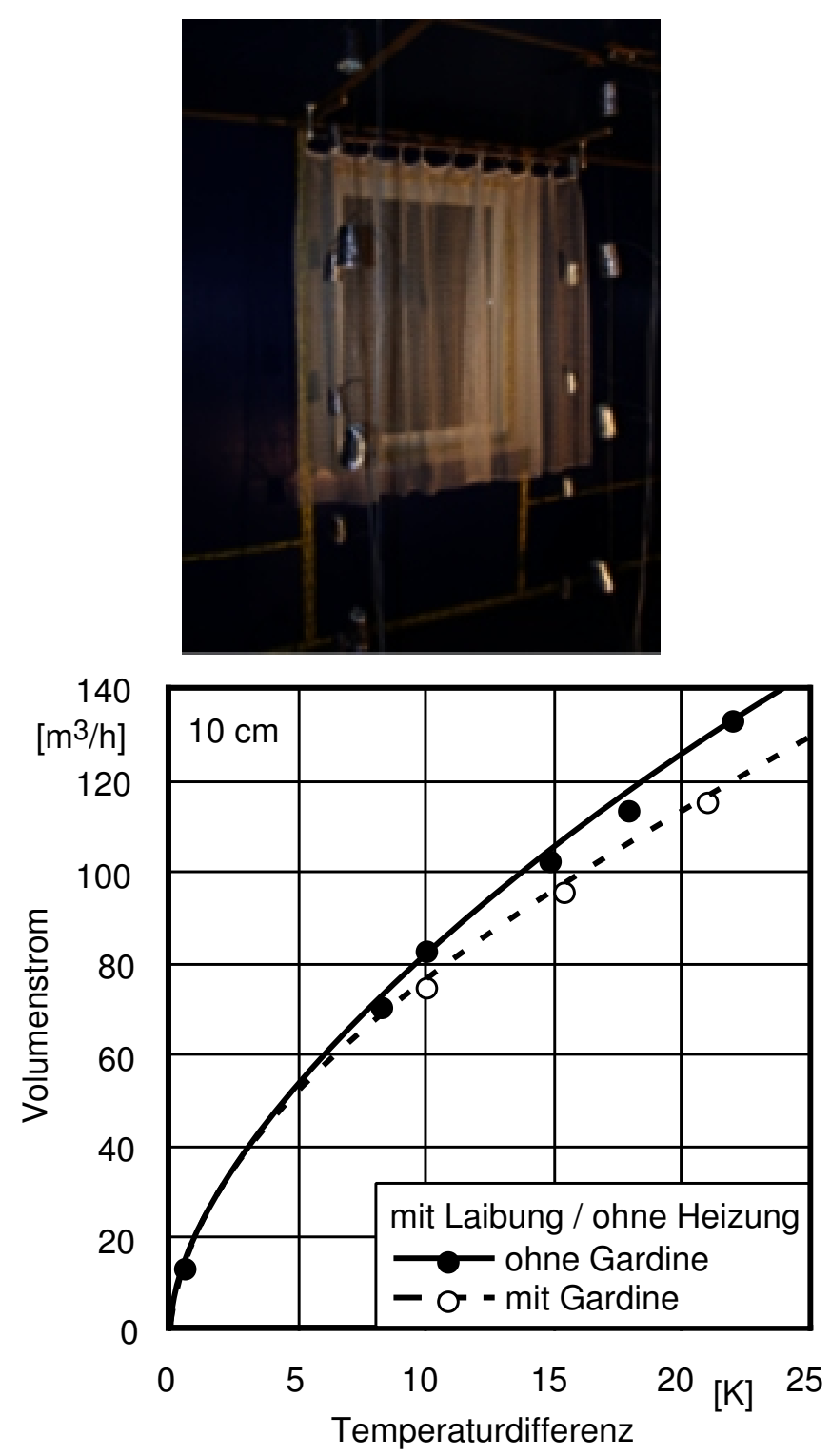

Bild 17: Zuluftvolumenstrom unter Berücksichtigung einer Gardine (Kippweite $10 \mathrm{~cm}$ ). 


\subsubsection{Vergleich mit Ansätzen aus der Literatur}

Die Messergebnissen für die Varianten 'ohne Laibung / mit Heizung' werden für alle drei Kippweiten mit den in Kapitel 3 vorgestellten Ansätzen aus der Literatur verglichen (Bild 18). Die Variante 'mit Laibung / mit Heizung' ist ergänzend aufgeführt. Das obere Bild zeigt die Ergebnisse für die Kippweite von $10 \mathrm{~cm}$, das mittlere Bild die für $6 \mathrm{~cm}$, und im unteren Bild sind die Ergebnisse für $3 \mathrm{~cm}$ Kippweite dargestellt.

Für die Kippweiten von $6 \mathrm{~cm}$ und $10 \mathrm{~cm}$ liegen die Messergebnisse der Variante 'ohne Laibung / mit Heizung' zwischen den berechneten Volumenströmen mit den Ansätzen Daler und Maas, d.h. mit dem Ansatz Daler wird bei vergleichbaren Randbedingungen der Volumenstrom um ca. $10 \% / 17 \%$ zu groß, mit dem Ansatz Maas um rund $27 \% / 22 \%$ zu gering berechnet.

Die Volumenströme für Variante 'ohne Laibung / mit Heizung' berechnet nach Weber $1 / 2$ betragen ca. $63 \%$ bzw. ca. $37 \%$ der Messwerte für die Kippweite von 10 cm und ca. $70 \%$ bzw. ca. $44 \%$ für die Messwerte der Kippweite von 6 cm.

Bei der Kippweite von $3 \mathrm{~cm}$ weist nur der Ansatz von Daler tendenziell eine Übereinstimmung mit den Messwerten auf. Die Ansätzen von Maas und Weber führen zu deutlich geringeren Volumenströmen, die in der Größenordnung von $45 \%$ bis $73 \%$ der Messwerte für die Variante 'ohne Laibung / mit Heizung' liegen.

Insgesamt zeigt sich, dass bis auf Daler mit allen Ansätzen für die Variante 'ohne Laibung / mit Heizung' zu geringe Volumenströme bestimmt werden. Dies gilt bis auf eine Ausnahme auch für die Variante 'mit Laibung / mit Heizung'. 


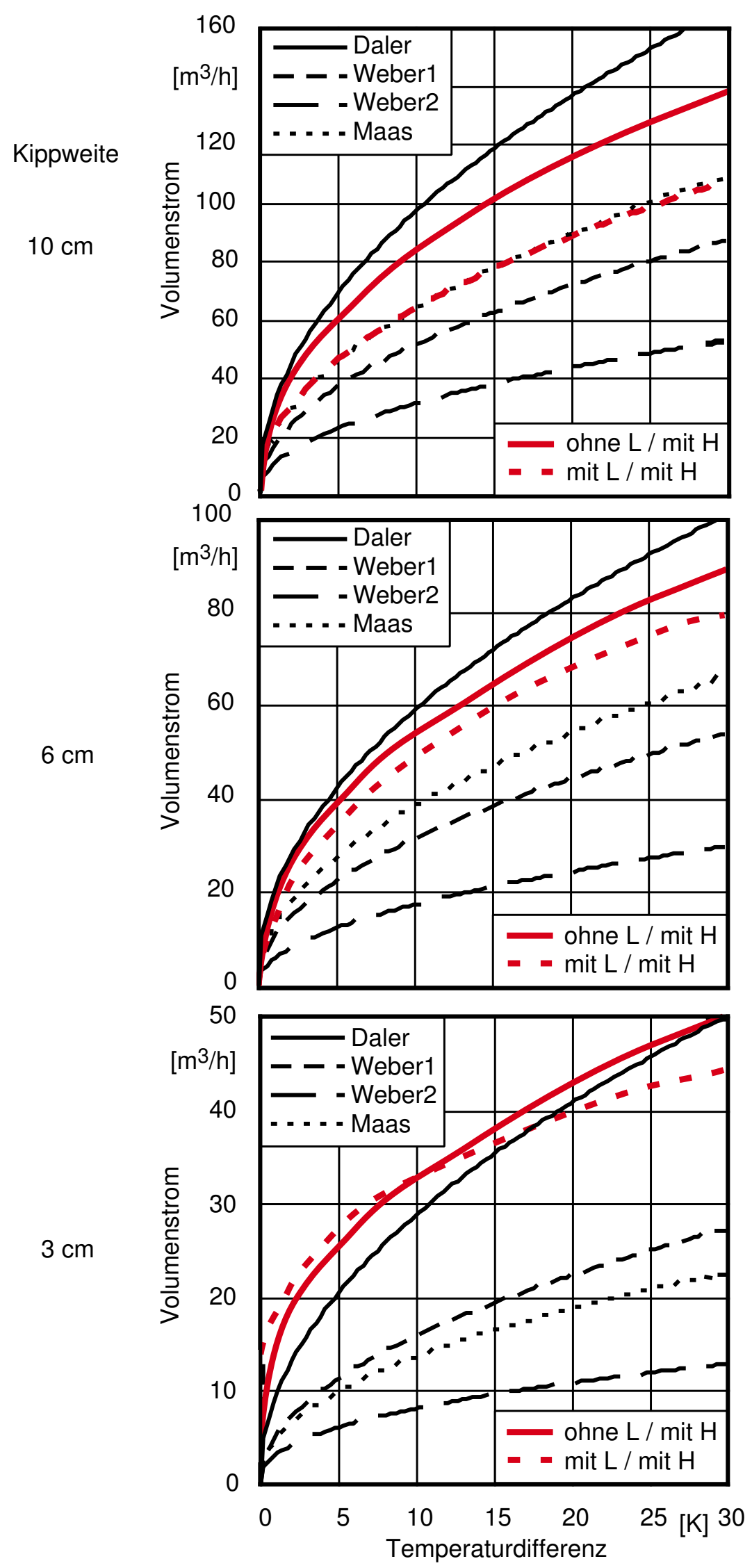

Bild 18: Vergleich der Messwerte mit den Ansätzen nach Daler, Weber und Maas. 


\subsection{Temperaturverteilung}

Während der Untersuchungen zur Bestimmung des Zuluftvolumenstroms erfolgt bei einigen Varianten eine detaillierte Betrachtung der Temperaturverteilung im Modellraum. Neben dem zeitlichen Verlauf der Raumtemperaturen soll auch der Lenkungseinfluss von Laibung und Heizung auf die Luftströmung ermittelt werden.

Die Aufnahme der Temperaturverteilung im Modellraum erfolgt für alle drei Kippweiten und jeweils die Varianten 'ohne Laibung / ohne Heizung', 'mit Laibung / ohne Heizung' und 'mit Laibung / mit Heizung'. Wegen der Übersichtlichkeit werden im folgenden Text nur die Lufttemperaturen der Varianten mit der Kippweite von $10 \mathrm{~cm}$ dargestellt und diskutiert. Alle anderen Ergebnisse können den Anhängen 12.2 bis 12.4 entnommen werden. Die Positionen der einzelnen Temperaturfühler sind Bild 9 zu entnehmen.

\subsubsection{Variante 'ohne Laibung / ohne Heizung'}

Für die Variante 'ohne Laibung / ohne Heizung' sind die Luftemperaturverläufe in Bild 19 dargestellt. Die Temperaturen der Messstellen A/B werden mit den anderen Varianten zusammengefasst (Bild 22). Die mittlere Temperaturdifferenz zwischen Modellraum und Labor beträgt $17 \mathrm{~K}$ und es wird ein Zuluftvolumenstrom von 138 $\mathrm{m}^{3} / \mathrm{h}$ bestimmt.

In Bild 19 sind die Temperaturverläufe für die Lufttemperaturen an den Positionen $\mathrm{C} / \mathrm{D} / \mathrm{E}, \mathrm{H} / \mathrm{I} / \mathrm{J}$ und M/N/O über der Versuchsdauer aufgetragen. Die Fensteröffnungsdauer ist eingetragen. Bevor das Fenster geöffnet wird, erfolgt die Injektion des Tracergases. Der Temperaturabfall ist darauf zurückzuführen, dass die Heizung während der Gaszugabe und -verteilung entfernt ist. Nach dem Schließen des Fensters ist deutlich zu erkennen, dass infolge der Ventilatoren die Luft gut durchmischt wird und eine homogene Temperatur aufweist.

Die Temperaturverläufe für die verschiedenen Positionen der Lufttemperaturen sind sehr ähnlich. Zu Beginn der Messung liegt eine homogene Temperaturverteilung vor. Alle Sensoren zeigen annährend $39^{\circ} \mathrm{C}$. Nach Beendigung der Messung - Fenster wird geschlossen und die Ventilatoren werden angestellt - ist ebenfalls deutlich zu erkennen, dass die Fühler nach einer kurzen Zeit - ca. 6 min - wieder eine einheitliche Temperatur aufweisen (ca. 35,3 ${ }^{\circ} \mathrm{C}$ ). Während der Messung bildet sich eine Temperaturschichtung aus, die zwar über die Raumbreite konstant ist, sich jedoch mit der Raumtiefe ändert.

Auffällig ist, dass nur die deckennahen Fühler in der Höhe 4 und 5 an allen Positionen einen linearen Temperaturabfall während der Lüftungsdauer aufweisen, während alle anderen Fühler in den ca. ersten 10 min einen exponentiellen Abfall zeigen und dann ebenfalls für die restliche Versuchsdauer in einen linearen Verlauf übergehen.

Der schnelle Temperaturabfall um ca. 3,5 K an allen bodennahen Temperaturfühlern in den ersten 10 min, deutet darauf hin, dass sich die kalte Luft hauptsächlich bodennah verteilt. Dort wird sie von der neu einströmenden kühleren Luft angehoben, 


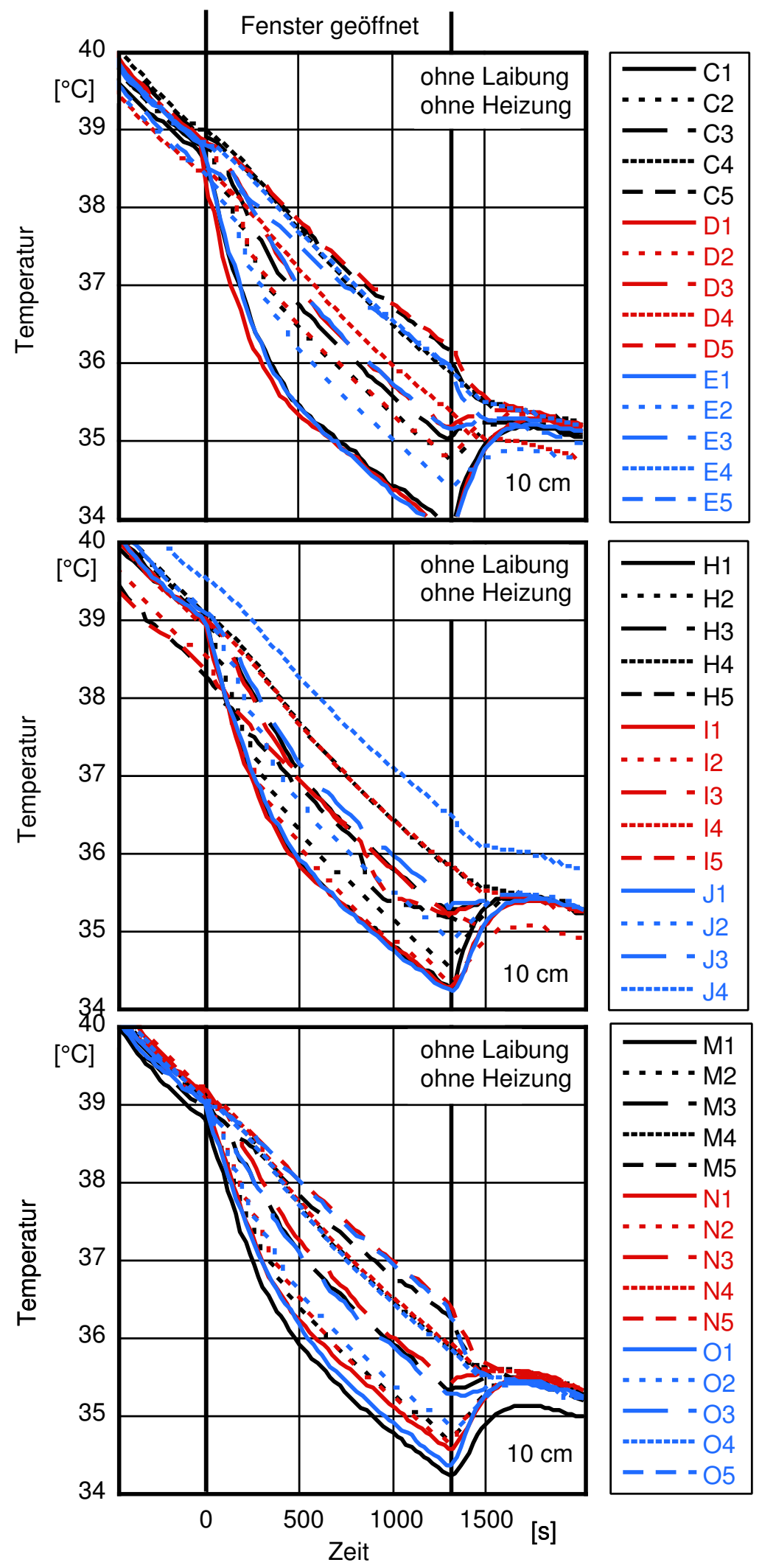

Bild 19: Zeitlicher Verlauf der Lufttemperaturen für die Variante 'ohne Laibung / ohne Heizung' an den Positionen C/D/E, H/I/J und $\mathrm{M} / \mathrm{N} / \mathrm{O}$ (Kippweite $10 \mathrm{~cm}$ ). 
so dass sich nach und nach eine konstante Temperaturschichtung ausbildet. Das gesamtes Temperaturniveau nimmt im Laufe der Zeit linear ab, d.h. die Raumluft kühlt stetig in Form einer Temperaturschichtung ab. Der Temperaturgradient beträgt von oben nach unten im vorderen und hinteren Teil des Raumes ca. 2,5 K und im mittleren Bereich rund 1,8 K. Im mittleren Teil des Raumes müssen sich Strömungsformen ausbilden, die zu einer größeren Durchmischung führen.

Zum Zeitpunkt, an dem das Fenster geschlossen wird, liegt folgende Temperaturverteilung vor: ein leichter Temperaturanstieg von vorne nach hinten um ca. $0,2 \mathrm{~K} \mathrm{im}$ deckennahen und um ca. 0,5 K im bodennahen Bereich sowie von unten nach oben um rund 2,5 $\mathrm{K}$ im fensternahen und um ca. 2,0 K im fensterfernen Bereich. Die tiefsten Raumlufttemperaturen sind im fensternahen Bereich am Boden festzustellen, während die höchsten Lufttemperaturen an der dem Fenster gegenüberliegenden Seite an der Decke auftreten.

\subsubsection{Variante 'mit Laibung / ohne Heizung'}

Für die Variante ' $10 \mathrm{~cm}$ Kippweite, mit Laibung / ohne Heizung' beträgt die mittlere Temperaturdifferenz zwischen Modellraum und Labor 15,5 K und der Zuluftvolumenstrom wird zu $107 \mathrm{~m}^{3} / \mathrm{h}$ bestimmt.

Prinzipiell verläuft die zeitliche Veränderung der Temperaturschichtung analog der Variante 'ohne Laibung / ohne Heizung' (Bild 20). Ein Unterschied tritt nur an den bodennahen Positionen D1/I1 auf. Diese Positionen befinden sich in der Mitte des Raumes. Sie weisen deutlich tiefere Lufttemperaturen auf, als bei der Variante 'ohne Laibung'. Dies wird auf einen Lenkungseffekt der Laibung zurückgeführt. Die Luft kann infolge der Laibung nicht seitlich des Fensters einströmen, sondern sie wird in die Mitte des Raumes gelenkt, d.h. die tiefste Temperatur ist mittig und bodennah vor dem Fenster zu lokalisieren. Die höchsten Temperaturen befinden sich wie bei der Variante 'ohne Laibung' deckennah an der dem Fenster gegenüberliegenden Wand.

Zum Zeitpunkt, an dem das Fenster geschlossen wird, liegt folgende Temperaturverteilung vor: ein leichter Temperaturanstieg von vorne nach hinten um ca. 0,3 K im deckennahen und um ca. $1 \mathrm{~K}$ im bodennahen Bereich sowie von unten nach oben um ca. $3 \mathrm{~K}$ im fensternahen und um ca. 2,5 K im fensterfernen Bereich.

\subsubsection{Variante 'mit Laibung / mit Heizung'}

Die mittlere Temperaturdifferenz für die Variante '10 cm Kippweite, mit Laibung / mit Heizung' beträgt zwischen Modellraum und Labor 18,6 K und es wird ein Zuluftvolumenstrom von $78 \mathrm{~m}^{3} / \mathrm{h}$ bestimmt. Der Heizkörper befindet sich während der Messung unterhalb des Fensters. Die aufgezeichneten Temperaturverläufe der Lufttemperaturen sind Bild $21 \mathrm{zu}$ entnehmen.

Im Vergleich zu den Varianten 'ohne Heizung' besteht bei der Variante 'mit Heizung' schon zu Beginn der Messung im Raum eine deutliche Temperaturschichtung 

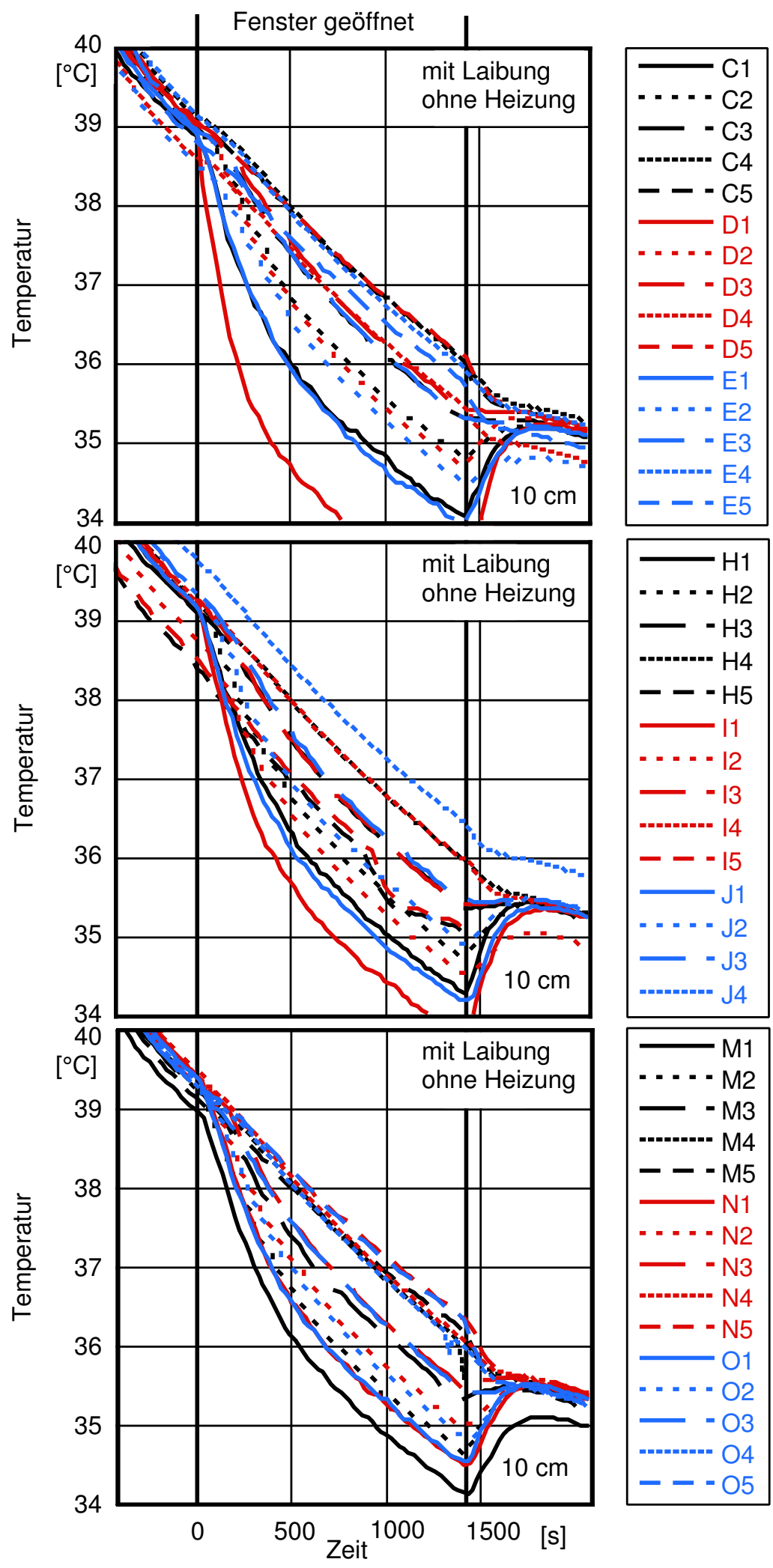

$--\mathrm{H} 5$
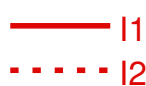

$-\quad-13$

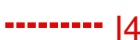

$--15$
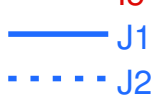

- - J3

45
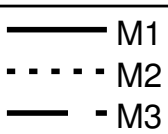

-+1+-1+" M4
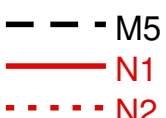

- $-\mathrm{N} 3$

-.-.-.- N4

$--\mathrm{N} 5$
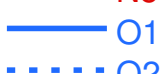

$-\quad-03$

-ே+m-1- 04

Bild 20: Zeitlicher Verlauf der Lufttemperaturen bei der Variante 'mit Laibung / ohne Heizung' an den Positionen C/D/E, H/I/J und M/N/O (Kippweite $10 \mathrm{~cm}$ ). 
von unten nach oben mit zunehmenden und von vorne nach hinten mit abnehmenden Lufttemperaturen. An den fensternahen seitlichen Temperaturfühlern C5/E5, die $20 \mathrm{~cm}$ von der Decke entfernt sind, ist der Einfluss des Heizkörpers deutlich zu registrieren. Diese Temperaturen liegen im Schnitt $2 \mathrm{~K}$ höher als die der nächst tieferen Ebene. Im mittleren und hinteren Bereich des Raumes nimmt dieser Temperaturvorsprung soweit ab, dass im hinteren Bereich die zwei obersten Lufttemperaturebenen die gleiche Temperatur aufweisen. Auffällig ist, dass nach dem Öffnen des Fensters die Lufttemperaturen der deckennahen Positionen C5 und E5 innerhalb von rund 10 Minuten um ca. $2 \mathrm{~K}$ fallen. Im gleichen Zeitraum sinken die bodennahen Temperaturen (C1/D1) nur um maximal ca. 1,5 K, welches im Vergleich mit den Varianten 'ohne Heizung' sehr wenig ist. Dies deutet darauf hin, dass die einund ausströmende Luft von der Heizung deutlich beeinflusst wird.

Der gesamte Temperaturverlauf stellt sich für die Variante 'mit Heizung' anders als für die Varianten 'ohne Heizung' dar. Bei dem Vorhandensein einer Heizung nimmt die Temperatur in allen Raumhöhen in den ersten 10 min exponentiell ab und bleibt für die weitere Messdauer relativ konstant, d.h. eine annähernd konstante Temperaturschichtung stellt sich ein. Der Temperaturgradient im vorderen Bereich beträgt rund 3,5 K, im mittleren und im hinteren Bereich ca. $3 \mathrm{~K}$. Die tiefsten Temperaturen im Modellraum sind bodennah an der dem Fenster gegenüberliegenden Seite zu verzeichnen. Über dem Fenster sind die Lufttemperaturen am höchsten.

Insgesamt kann festgestellt werden, dass während der Messung von vorne nach hinten eine Reduktion der Temperaturen stattfindet, im deckennahen Bereich um ca. $2 \mathrm{~K}$, im Bereich des Fußbodens um ca. $1 \mathrm{~K}$. Von oben nach unten beträgt die Temperaturabnahme im fensternahen Bereich ca. 3,5 K und im fensterfernen ca. 2,5 $\mathrm{K}$.

\subsubsection{Luftemperaturen im fensternahen Bereich}

Für alle Varianten sind die Lufttemperaturen an den fensternahen Positionen A und B Bild $22 \mathrm{zu}$ entnehmen. Im oberen Teil des Bildes sind die Verläufe für die Variante 'ohne Laibung / ohne Heizung', in der Mitte für die Variante 'mit Laibung / ohne Heizung' und im unteren Teil für die Variante 'mit Laibung / mit Heizung' dargestellt.

Für die Variante 'ohne Laibung / ohne Heizung' zeigt sich, dass der Temperaturverlauf in diesem Bereich den anderen Lufttemperaturen (Bild 19) sehr ähnlich ist. Dies bedeutet, dass die einfallende Luft nur seitlich des Fensters einströmt und keine Strömungskomponente senkrecht zum Fenster aufweist.

Der Lenkungseffekt einer Laibung wird durch die Lufttemperaturen an den Positionen A/B bei der Variante 'mit Laibung / ohne Heizung' bestätigt. Die Lufttemperaturen in der unteren Hälfte der Raumhöhe liegen z.T. deutlich niedriger als bei der Variante 'ohne Laibung' bzw. den Lufttemperaturen an den bodennahen Positionen C/D/E (Bild 20). Die einströmende Luft wird kanalisiert und strömt nicht nur seitlich sondern auch senkrecht zum Fenster in den Modellraum. Die Temperaturverläufe weisen auf eine starke Verwirbelung im fensternahen Bereich hin. 

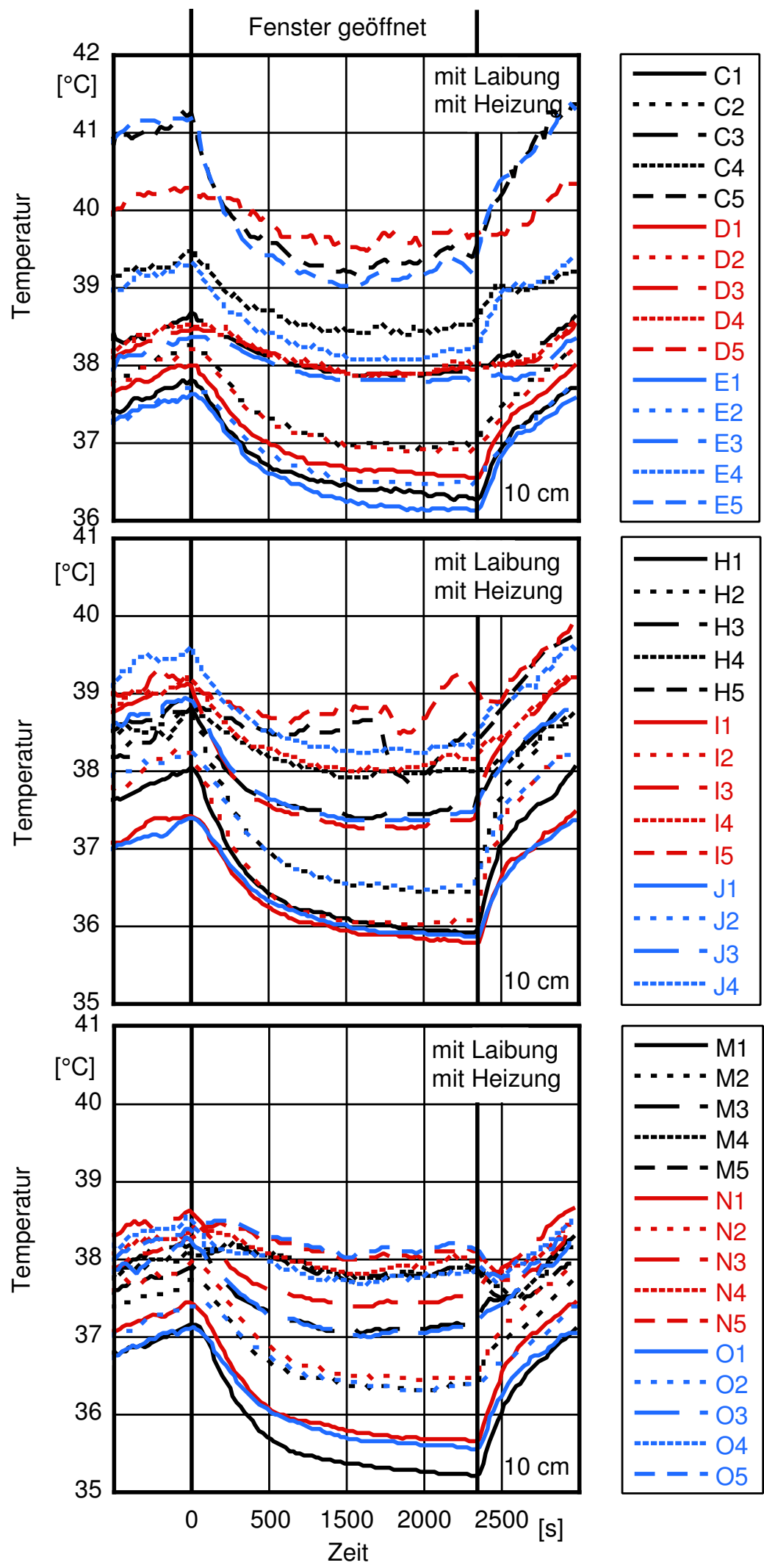

Bild 21: Zeitlicher Verlauf der Lufttemperaturen bei der Variante 'mit Laibung / mit Heizung' an den Positionen C/D/E, H/I/J und $\mathrm{M} / \mathrm{N} / \mathrm{O}$ (Kippweite $10 \mathrm{~cm}$ ). 
Der Temperaturverlauf an den Positionen A/B für die Variante 'mit Laibung / mit Heizung' zeigt, dass die deckennahen Temperaturen (A5/B5) deutlich höher liegen als bei den anderen entsprechenden Lufttemperaturen im Raum (Bild 21). Dies ist naheliegend, da in der Nähe auch die Heizung steht.

\subsubsection{Zusammenfassung der Temperaturverläufe}

Die gemessenen Temperaturverläufe zeigen für alle Varianten, dass die kalte Luft sich am Boden sammelt und durch neue einströmende Luft nach oben gehoben wird. Hieraus entsteht eine Temperaturschichtung im Raum, die über die Raumbreite weitgehend konstant ist und sich mit der Raumtiefe ändert. Während bei den Varianten 'ohne Heizung' die tiefste Temperatur im fensternahen Bereich am Boden zu finden ist, stellt sich die niedrigste Temperatur bei der Variante 'mit Heizung' bodennah an der dem Fenster gegenüberliegenden Wand ein. Die höchsten Temperaturen im Raum sind jeweils diagonal gegenüber anzutreffen.

Die Lufttemperaturen zeigen für alle drei Varianten in den ca. ersten 10 min Öffnungszeit einen exponentiellen Abfall. Während bei den Varianten 'ohne Heizung' der Temperaturabfall nach rund $10 \mathrm{~min}$ in einen linearen Verlauf übergeht und über die gesamte restliche Lüftungsdauer anhält, stellen sich bei der Variante 'mit Heizung' überall relativ konstante Temperaturen ein.

Insgesamt sind für die Varianten 'ohne Heizung' eine deutliche Verminderung der Lufttemperaturen während der Fensteröffnungszeit festzustellen. Die stärkste Reduktion der Temperatur ist bodennah zu verzeichnen. Die Temperaturabnahme wird häufig dazu führen, dass das Fenster nach einer relativ kurzen Zeit wieder geschlossen wird, da es dem Nutzer insbesondere an den Füßen zu kalt wird.

Ist eine Heizung vorhanden, ist der Temperaturabfall nicht so ausgeprägt. Dies kann dazu führen kann, dass das Fenster länger als für einen als notwendig erachteten Luftwechsel geöffnet bleibt.

Bei der Variante 'mit Laibung / ohne Heizung' ist ein deutlicher Lenkungseffekt durch die Laibung festzustellen. Die bodennahen Temperaturen mittig vor dem Fenster sind niedriger als für den Fall 'ohne Laibung'. 


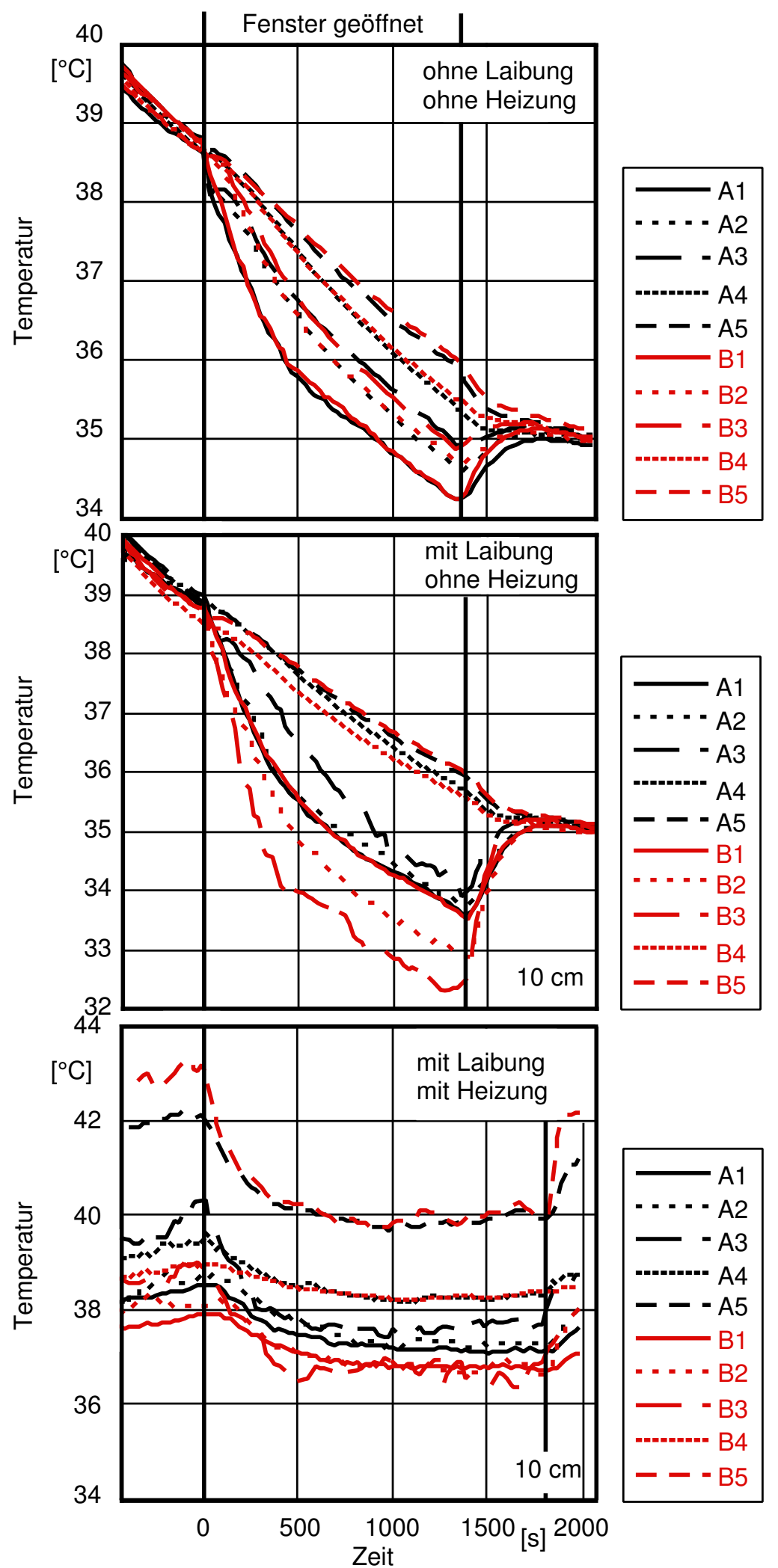

Bild 22: Zeitliche Temperaturverläufe im Bereich des Fensters für alle Varianten (Positionen A/B, Kippweite $10 \mathrm{~cm}$ ). 


\section{Numerische Simulation}

\subsection{Zur Simulation von Auftriebsströmungen}

Die Anwendung eines Computational Fluid Dynamics (CFD)-Programms zur Simulation von auftriebsinduzierten Strömungen findet in der Wissenschaft und in der Praxis eine immer größer werdende Verbreitung. Steigende Rechnerleistungen und leistungsstarke Programme erlauben es, immer komplexere Problemstellungen zu simulieren.

In [14] wird der thermisch induzierte Luftwechsel über einem Kippfenster als dreidimensionales Modell simuliert. Die über das Kippfenster rechnerisch bestimmten Massenströme werden zur Ableitung eines Korrekturfaktors für ein theoretisches Modell zur Bestimmung von Volumenströmen über ein Kippfenster herangezogen (Abschnitt 3.4 und 3.5).

Unter dem Gesichtspunkt der thermischen Behaglichkeit wird in [59] der Luftaustausch über ein Kippfenster mit CFD simuliert und messtechnisch untersucht. Der Schwerpunkt liegt auf der Bestimmung der Luftgeschwindigkeiten im Raum. Die Ergebnisse zeigen, dass sich die Raumluftströmung qualitativ mit CFD nachbilden lässt. Eine Temperaturschichtung mit zur Decke ansteigenden Temperaturen sowie eine abnehmende Luftgeschwindigkeit in Bodennähe vom fensternahen zum fensterfernen Bereich, wird durch Versuch und Simulation gleichermaßen ermittelt. Schwierigkeiten gibt es bei der Ableitung einer quantitativen Aussage aus der Strömungssimulation. Im untersuchten Fall treten die größten Abweichungen zwischen Simulation und Messung für die Luftgeschwindigkeiten im Bereich des Fensters auf. Es wird darauf hingewiesen, dass die Aussagekraft von Simulationsergebnissen ohne den Abgleich mit Messwerten eingeschränkt ist. Fehlen Messwerte, so ist die Gefahr groß ungenaue bzw. sogar falsche Ergebnisse zu erhalten.

Schwerpunkt der Arbeit von [27] liegt auf der Messung und Simulation einer thermisch induzierten Auftriebsströmung in einer Modellbox mit den Maßen 0,49 x 0,74 x 1,10 m (H x B x L). Der Einfluss von verschiedenen Gitterstrukturen, Turbulenz- und Strahlungsmodellen, Betrachtung von Symmetrieebenen und zwei- bzw. dreidimensionalen Modellen wird untersucht. Als Ergebnis dieser Studie wird für auftriebsbestimmte Strömungen empfohlen, den Einsatz von zweidimensionalen Modellen und Symmetrieebenen zu vermeiden, instationäre Berechnungen mit kurzen Zeitschritten durchzuführen sowie aufwändige Wand- und Strahlungsmodelle zu verwenden.

Auftriebsströmungen in einem dreigeschossigen Treppenhaus werden in [43] beschrieben. In der Arbeit wird darauf hingewiesen, dass Auftriebsströmungen mit CFD schwierig zu modellieren sind. Die Hauptgründe hierfür sind die geringen treibenden Kräfte, welche zu numerischen Instabilitäten führen, die Wahl des Turbulenzmodelles sowie die Definition der Randbedingungen, welche i.d.R. weder exakt noch vollständig vorliegen. Die Ergebnisse der CFD-Simulationen zeigen jedoch recht gute Übereinstimmungen mit den punktuell bestimmten Temperaturen und Luftgeschwindigkeiten sowie der gemessenen Tracergas-Konzentration im realen Treppenhaus. 
Die Einsatzmöglichkeiten von CFD zur Simulation von thermisch induzierten Auftriebsströmungen sind vielfältig, jedoch müssen die Ergebnisse kritisch hinterfragt werden. Schon geringe Änderungen an den Randbedingungen und Simulationsparametern können statt zu brauchbaren Resultaten zu völlig unrealistischen Ergebnissen führen [27].

\subsection{Grundlagen}

CFD-Simulationsprogramme bieten die Möglichkeit, mit einer feinen zeitlichen und räumlichen Auflösung Fluidströmungen zu modellieren. Grundlage der numerischen Berechnung von Strömungen bilden die Erhaltungsgleichungen für Masse, Impuls und Energie. Eine vertiefte Darstellung der Grundgleichungen für die Strömungsmechanik finden sich z.B. in [41] und [61]. Die Turbulenzmodellierung ist z.B. in [21] beschrieben.

Eine Strömung kann zwei grundsätzlich verschiedene Zustände annehmen. Eine laminare Strömung liegt vor, wenn die Strömung in parallelen Schichten erfolgt. Ist die Strömung turbulent, ist ein Austausch zwischen den einzelnen Schichten erkennbar und es treten wirbelartige Zufallsbewegungen auf. Ob eine Strömung als laminar oder turbulent anzusehen ist, kann mit Hilfe dimensionsloser Kennzahlen ermittelt werden. Bei einer erzwungenen Konvektion ist die Reynolds-Zahl Re die entscheidende Kennzahl

$$
R e=\frac{w L}{\nu} \quad[-]
$$

mit w, der charakteristischen Geschwindigkeit - z.B. mittlere Geschwindigkeit in einem Rohrquerschnitt - und L, der charakteristischen Länge - z.B. Rohrdurchmesser - und der kinematischen Viskosität $\nu$. Strömt ein Medium z.B. durch ein kreisrundes Rohr, liegt eine laminare Strömung vor, wenn $\operatorname{Re}<2300$ ist. Bei größeren Reynolds-Zahlen spricht man von einer turbulenten Strömung. Die Reynolds-Zahl kennzeichnet das Verhältnis von Trägheitskraft $F_{T} \sim w^{2} / L$ zur Reibungskraft $F_{R} \sim(\nu w) / L^{2}$.

Für die freie Konvektion spielt die Reynolds-Zahl keine Rolle, da keine charakteristische Geschwindigkeit gegeben ist. Stattdessen ist eine Kennzahl, die den charakteristischer Wert des hydrostatischen Auftriebs $F_{A} \sim g \beta|\Delta T|$ beinhaltet, anzusetzen, wobei $\beta$ (=1/T für Gase) der thermische Ausdehnungskoeffizient ist [44]. Für die Beschreibung der natürlichen Konvektion wird die Grashof-Zahl Gr

$$
G r=\frac{g \beta \Delta T L^{3}}{\nu^{2}} \quad[-]
$$

herangezogen. Sie beschreibt das Verhältnis von Auftriebskraft $F_{A}$ zur Reibungskraft $F_{R}$. Bei freien Konvektionsströmungen, in denen Auftrieb, Zähigkeit und Wärmeleitung eine Rolle spielen werden die Grashof-Zahl Gr und die Prandtl-Zahl Pr zur Rayleigh-Zahl Ra kombiniert:

$$
R a=\operatorname{Gr} \operatorname{Pr} \quad[-]
$$


Während die Reynolds-Zahl und die Grashof-Zahl von den geometrischen, dynamischen und den thermodynamischen Parametern des jeweiligen Problems abhängen, ist die Prandtl-Zahl eine reine Stoffgröße:

$$
\operatorname{Pr}=\frac{c_{p} \mu}{\lambda} \quad[-]
$$

Sie stellt das Verhältnis aus kinematischer Zähigkeit $\nu=\mu / \rho$ und Temperaturleitfähigkeit $a=\lambda /\left(\rho c_{p}\right)$ dar. Die Prandtl-Zahl kann als Verhältnis des molekularen Impuls- und Wärmetransports interpretiert werden [13]. Für Luft ist $\operatorname{Pr}=0,7$ ein Näherungswert, der praktisch für alle Temperaturen oberhalb von $-50^{\circ} \mathrm{Cverwendet}$ werden kann [44].

Die Rayleigh-Zahl ist für die Stabilität natürlicher Konvektionsströmungen maßgebend. Eine laminare Strömung liegt vor, wenn $\mathrm{Ra}<10^{8}$ ist. Für $\mathrm{Ra}>10^{10}$ ist eine turbulente Strömung zu erwarten. Etwa zwischen $10^{8}$ und $10^{10}$ liegt ein Übergangsgebiet [44].

Für die im Rahmen dieser Arbeit beschriebenen Untersuchungen an einem Kippfenster mit den Kippweiten von $3 \mathrm{~cm}, 6 \mathrm{~cm}$ und $10 \mathrm{~cm}$ werden die jeweiligen um die Rahmenüberschneidung reduzierten Fensterhöhen als charakteristische Länge L eingesetzt und die entsprechenden Rayleigh-Zahlen für eine Temperaturdifferenz von $15 \mathrm{~K}-\mathrm{Ra} \approx 2 \cdot 10^{9}(10 \mathrm{~cm}), \mathrm{Ra} \approx 1 \cdot 10^{9}(6 \mathrm{~cm})$ und $\mathrm{Ra} \approx 4 \cdot 10^{8}(3 \mathrm{~cm})$ - erhalten. Die Rayleigh-Zahl für die Bedingungen im Raum ergibt sich mit der Raumhöhe von 2,5 m als charakteristischer Länge zu rund $1 \cdot 10^{10}$. Im Bereich des Fensters liegt keine ausgeprägte Strömungsform vor, während man im Raum von einer annähernd turbulenten Strömung ausgehen kann.

Die Archimedes-Zahl Ar

$$
A r=\frac{G r}{R e^{2}} \quad[-]
$$

spiegelt das Verhältnis von freier und erzwungener Konvektion bzw. Auftriebskraft $F_{A}$ zur Trägkeitskraft $F_{T}$ [60] wieder. Wird Ar $\gg 1$, dominiert die freie Konvektion, bei $\mathrm{Ar} \ll 1$ überwiegt die erzwungene Konvektion und bei $\mathrm{Ar} \approx 1$ sind beide Effekte signifikant [12]. Aus den Simulationen kann für den Modellraum $\mathrm{Ar} \approx 60$ bestimmt werden, d.h. es liegt eine freie Konvektion vor. 


\subsection{Erstellung des Modells}

Der Modellraum wird im Maßstab 1:1 mit 2,5 x 3,6 x 5,0 m (H x B x L) abgebildet. Die Modellraumwände sowie der Fensterflügel werden als Fläche modelliert. Das Volumen des Labors ist in Anbetracht der zu erwartenden Anzahl an Knoten, auf einen Streifen mit der Breite des Modellraumes reduziert. Bild 23 zeigt eine Skizze der Geometrie des CFD-Modells.

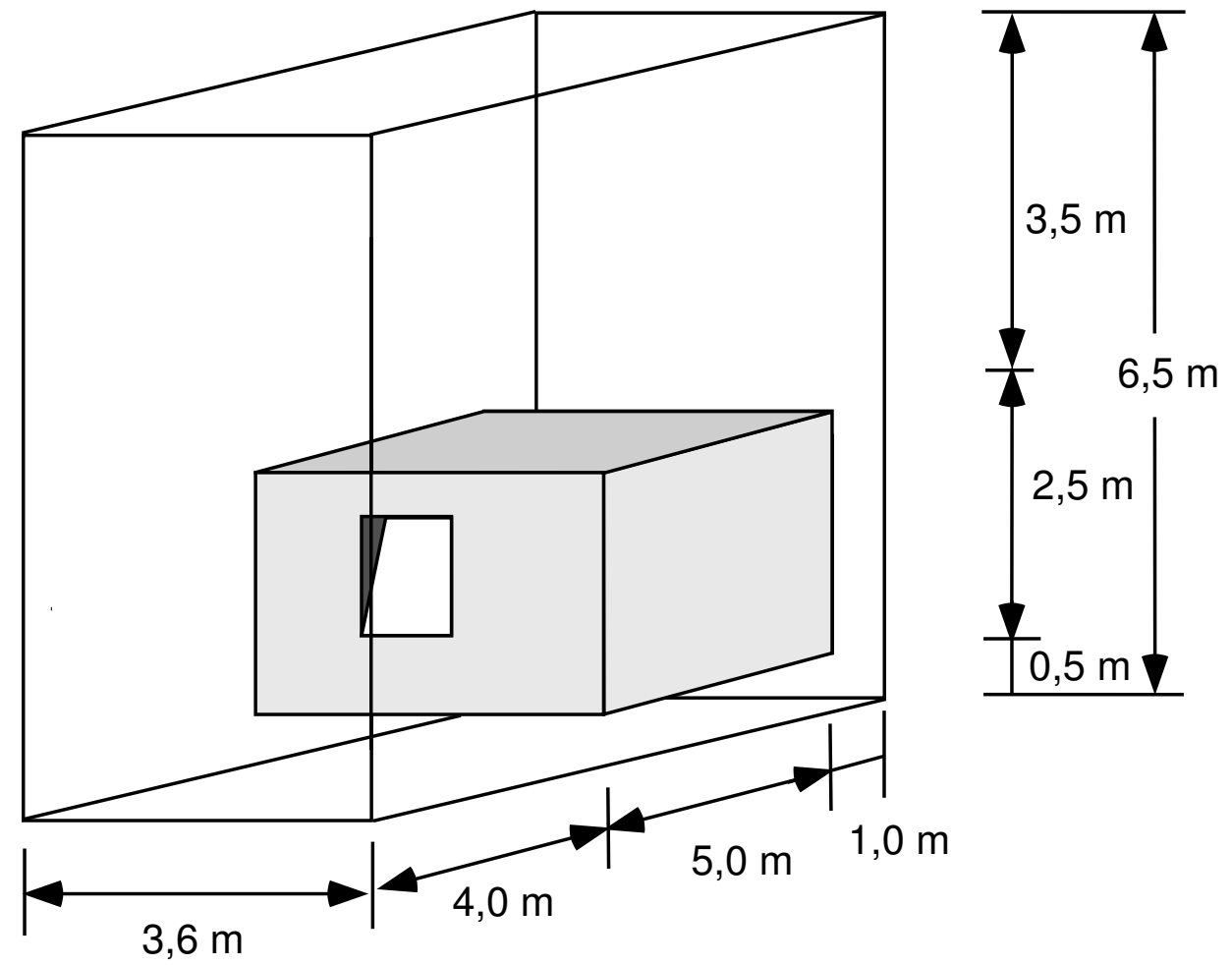

Bild 23: Skizze der Geometrie des CFD-Modells.

Die Vernetzung erfolgt mit einem Tetraeder-Gitter, wobei der Modellraum feiner vernetzt ist als das umgebende Labor. Die Geometrie und die Berechnungsgitter werden mit dem Programm Gambit 2.04 [21] erstellt. In dem verwendeten Simulationsprogramm Fluent 6.0 wird in einem zweiten Schritt das Berechnungsgitter im Bereich des Kippfensters verfeinert. Anschließend beläuft sich die Gesamtanzahl der Knoten auf ca. 110000, wobei rund $60 \%$ der Knoten auf den Modellraum entfallen. In Bild 24 ist eine Seitenansicht des CFD-Gittermodells dargestellt. Das Berechnugsgitter in dem mit einem weißen Rechteck gekennzeichneten Bereich wird in Fluent nochmals verfeinert. Dabei werden die einzelnen Tetraeder in acht Zellen unterteilt. Die Gitterpunkte in der Öffnungsfläche des Kippfensters nach der Verfeinerung zeigt Bild 25. 


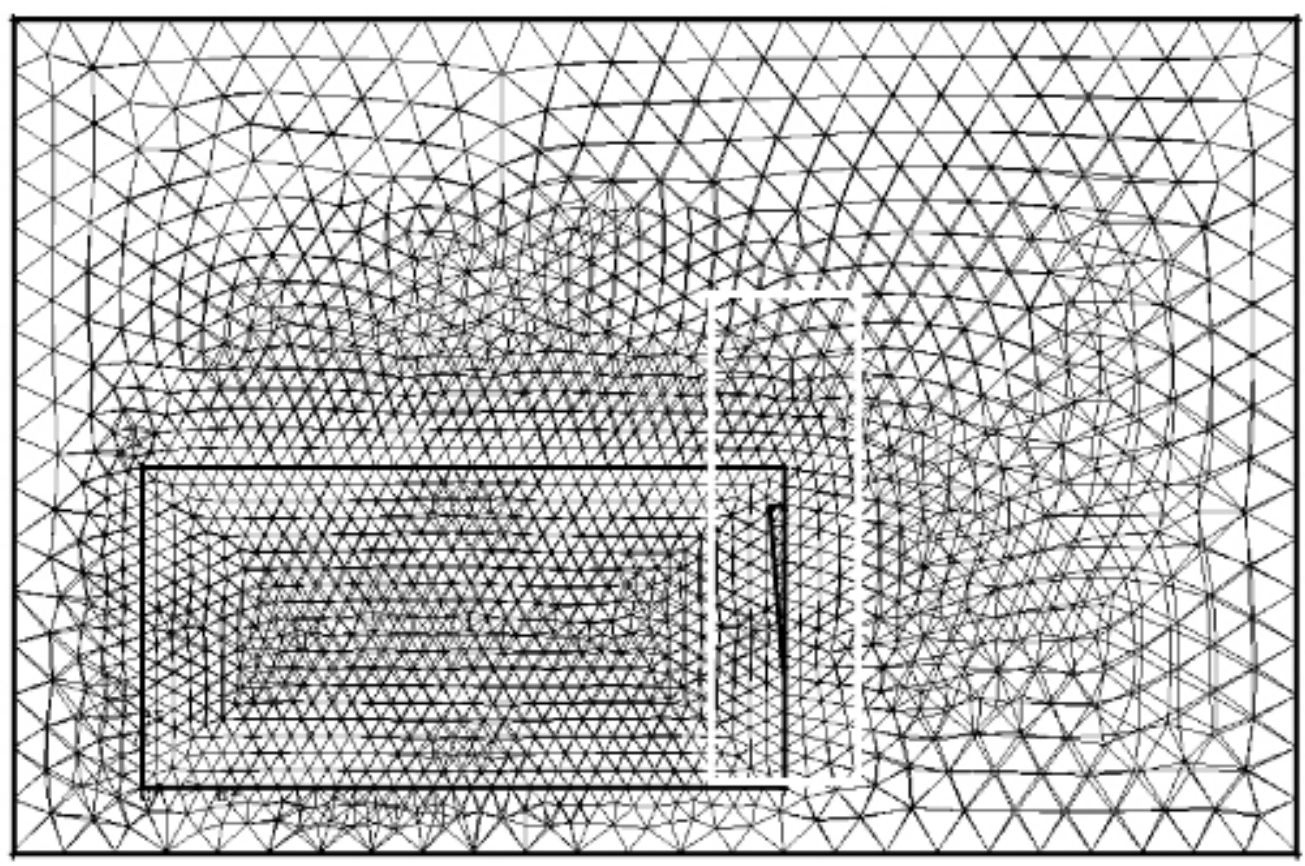

Bild 24: Seitenansicht des CFD-Modells mit Gitterpunkten. Das Gitter in dem weiß umrandeten Bereich wird nochmals verfeinert.

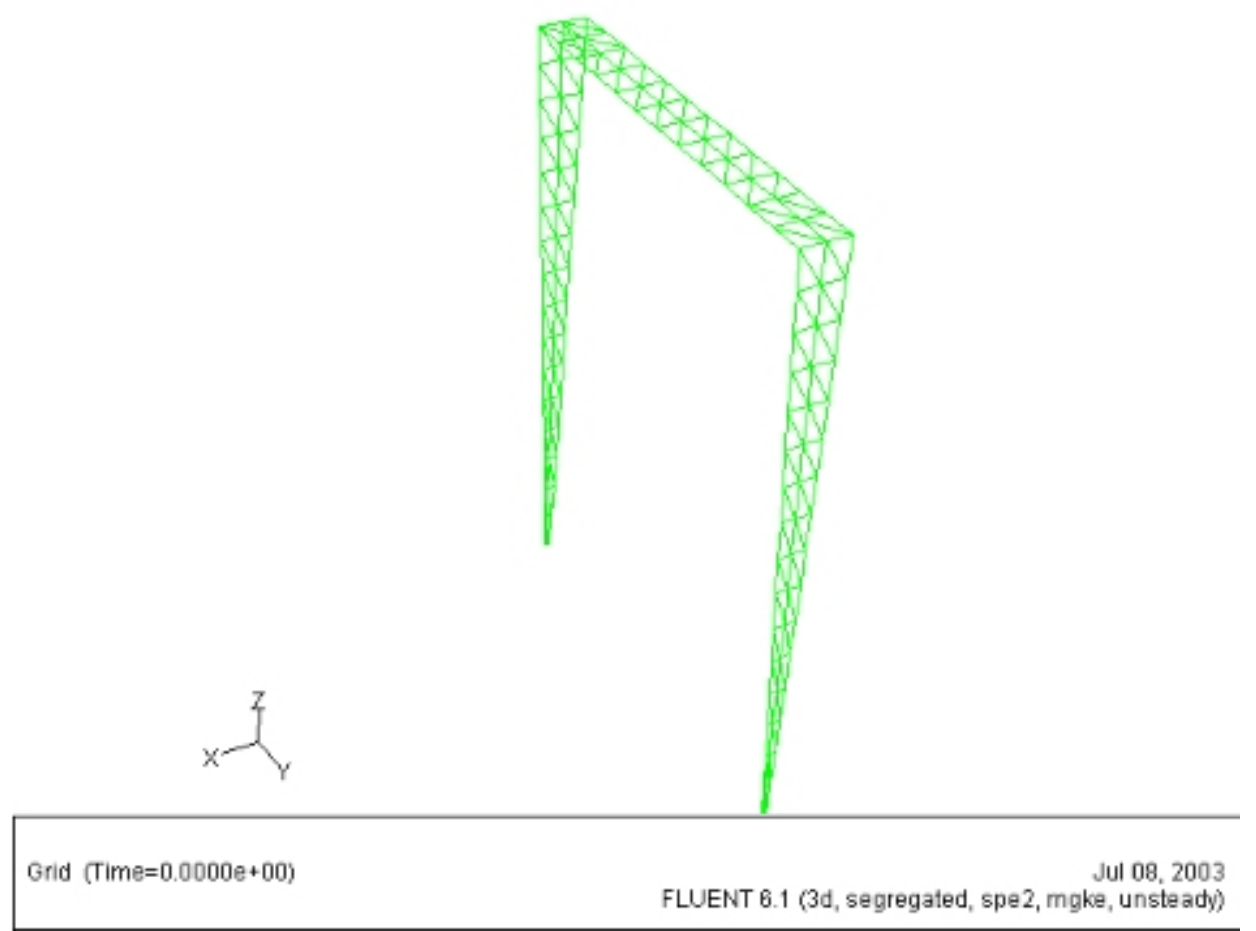

Bild 25: Gitterpunkte in der Ebene der Öffnungsfläche des Kippfensters nach der Verfeinerung. 


\subsection{Modellparameter}

Bei der Wahl der Randbedingungen fließen z.T. die Ergebnisse einer früheren Dissertation [27] am Fachgebiet Bauphysik ein. In dieser Arbeit hat sich gezeigt, dass es für die Berechnung von freier Konvektion vorteilhaft ist, mit dreidimensionalen Modellen, ohne Symmetrieebene und instationär zu rechnen. Die Luft kann als inkompressibles ideales Gas betrachtet werden. Eine gute Konvergenz ist mit kleineren Zeitschritten, als von Fluent vorgeschlagen wird, zu erwarten.

Als Turbulenz-Modell wird das Renormalization-Group (RNG) k- $\epsilon$-Modell eingesetzt. Das RNG k- $\epsilon$-Modell ist eine Weiterentwicklung des $\mathrm{k}-\epsilon$-Modells, welches auf den sog. Reynolds-averaged Navier-Stokes-Gleichungen (RANS) [21], beruht. Um Aussagen über turbulente Strömungen treffen zu können werden die in den Navier-Stokes-Gleichungen vorkommenden Größen nach der Reynolds-Mittelung [21] in einen zeitlichen Mittelwert und eine Schwankungsgröße, welche die zeitliche Abweichung der Größe von ihrem Mittelwert angibt, zerlegt.

Zur Bestimmung der Schwankungsgrößen sind im k- $\epsilon$-Modell zwei zusätzliche Gleichungen zu lösen. Zum einen muss eine Gleichung zur Bestimmung der turbulenten kinetischen Energie k - verantwortlich für die Entstehung der Turbulenz - und zum anderen eine Gleichung für die Dissipationsgeschwindigkeit der kinetischen Energie $\epsilon$ - verantwortlich für das Abklingen der Turbulenz - gelöst werden [21].

Das RNG k- $\epsilon$-Modell wird gewählt, da dies im Gegensatz zum k- $\epsilon$-Modell, auch für nicht voll turbulent ausgebildete Strömungen gültig ist [21]. In [43] und [31] wird ebenfalls das RNG k- $\epsilon$-Modell für auftriebsinduzierte Strömungen verwendet und beide Autoren halten das Modell für geeignet.

In Wandnähe führt der Reibungseinfluss zur Bildung einer Strömungsgrenzschicht. Die Geschwindigkeit von der freien Strömung wird an der Wandoberfläche auf den Wert Null reduziert. Unmittelbar an der Wand ist die Strömung laminar, weiter weg geht sie i.d.R. in eine turbulente Strömung über. Die wandnahen Strömungsverhältnisse werden von den o.g. Turbulenzmodellen nicht abgebildet, so dass hierfür z.B. spezielle semi-empirischen Wandfunktionen eingesetzt werden können. Diese Funktionen berücksichtigen sowohl den laminaren Bereich der Grenzschicht üblicherweise durch eine lineare Geschwindigkeitsverteilung angenähert - sowie den turbulenten Bereich, in dem die Geschwindigkeit im Allgemeinen logarithmisch modelliert wird [21].

Zur Beschreibung der wandnahen Strömungsverhältnisse wird die 'Standard-Wandfunktion' verwendet. Kriterium für die Gültigkeit der 'Standard-Wandfunktion' ist der dimensionslose Wandabstand $\mathrm{y}^{+}$. Er ist definiert als

$$
y^{+}=y \frac{u_{\tau}}{\nu}=\frac{\bar{u}(y)}{u_{\tau}} \quad[-]
$$

mit der Wandschubspannungsgeschwindigkeit $u_{\tau}=\sqrt{\tau_{W} / \rho}$ und der mittleren Strömungsgeschwindigkeit $\bar{u}(y)$ im Abstand y zur Wand [61, 21, 33]. Gleichung 25 
beschreibt die Geschwindigkeitsverteilung für die laminare Unterschicht. Für den vollturbulenten Bereich wird ein logarithmischer Ansatz eingesetzt.

Der logarithmische Ansatz der 'Standard-Wandfunktion' ist für $\mathrm{y}^{+}>30 \sim 60$ gültig. Er wird jedoch eingesetzt, sobald $\mathrm{y}^{+}>11,225$ ist. Für kleinere Werte gilt der lineare Ansatz (Gl. 25). Ein Wert von $\mathrm{y}^{+} \approx 30$ ist anzustreben [21].

Fluent bietet die Möglichkeit, $\mathrm{y}^{+}$-Werte anzuzeigen, und eine Überprüfung der modellierten Modellraumwände zeigt, dass $\mathrm{y}^{+}$in dem Bereich zwischen 20 und 60 liegt, wobei rund $80 \%$ der Flächen einen Wert von $\mathrm{y}^{+} \sim 30-40$ aufweisen.

Analog zu den Strömungsgrenzschichten werden auch die thermischen Grenzschichten mit der 'Standard-Wandfunktion' berechnet. Ein linearer Ansatz wird für die wandnahe Schicht verwendet, bei der die Wärmeleitung eine große Rolle spielt. Für turbulente Bereiche in denen turbulente Effekte die Wärmeleitung dominieren wird ein logarithmischer Ansatz eingesetzt [21]. Bei Gasströmungen sind die Dicke der Temperatur- und Strömungsgrenzschichten von gleicher Größenordnung [33].

Die Berücksichtigung des Strahlungsaustausches kann durch den Einsatz eines Strahlungsmodells erfolgen, jedoch wird die Rechenzeit infolge der zusätzlich zu lösenden Gleichungen erheblich erhöht. Aus diesem Grund wird ein Fall mit und ohne Verwendung eines Strahlungsmodells betrachtet, um den Einfluss der Strahlung auf das Ergebnis zu quantifizieren. Als Strahlungsmodell wird das Discrete-Ordinate (DO)Modell verwendet. Der Vergleich der Ergebnisse zeigt, dass unter Berücksichtigung der Strahlung ein um knapp $3 \%$ erhöhter Volumenstrom berechnet wird. Diese recht geringe Abweichung steht einer deutlich verlängerten Rechenzeit gegenüber, so dass für die durchgeführten Simulationen auf den Einsatz eines Strahlungsmodells verzichtet wird.

Für den Abgleich der Ergebnisse aus den CFD-Simulationen mit den Messwerten werden exemplarisch einige Varianten der messtechnisch untersuchten Kippstellungen simuliert. Der Zuluftvolumenstrom wird über eine 'rechnerische' TracergasMessung bestimmt. Zu Beginn der Simulation wird der Modellraum mit 100 ppm Distickstoffoxid initiiert und der Konzentrationsabfall über die Zeit protokolliert. Die 'rechnerische' Lüftungsdauer wird in Abhängigkeit von den Randbedingungen der Wandtemperaturen gewählt. Werden die Wandtemperaturen konstant gehalten (Wandtemperatur gleich Startzeit-Lufttemperatur), entspricht die 'rechnerische' der entsprechend messtechnischen Lüftungsdauer. Die Bestimmung des Zuluftvolumenstroms erfolgt nach Gl. 3. Bei variablen Wandtemperaturen, erfolgt ebenfalls die Auswertung nach Gl. 3, jedoch wird der maximal sich einstellende Volumenstrom bestimmt.

Bei den verwendeten Materialien werden folgende Parameter zugrunde gelegt:

$$
\text { Luft } \begin{aligned}
\rho & =1,225 \mathrm{~kg} / \mathrm{m}^{3}, \mathrm{c}_{\mathrm{p}}=1006,43 \mathrm{~J} /(\mathrm{kg} \mathrm{K}), \\
\lambda & =0,0242 \mathrm{~W} /(\mathrm{m} \mathrm{K}), \mu=0,017894 \mathrm{~g} /(\mathrm{m} \mathrm{s})[21] \\
\mathbf{N}_{2} \mathbf{O} \rho & =1,85 \mathrm{~kg} / \mathrm{m}^{3}, \mathrm{c}_{\mathrm{p}}=1040,61 \mathrm{~J} /(\mathrm{kg} \mathrm{K}), \\
\lambda & =0,017 \mathrm{~W} /(\mathrm{m} \mathrm{K}), \mu=0,020504 \mathrm{~g} /(\mathrm{m} \mathrm{s})[47]
\end{aligned}
$$

Dämmung $\rho=33 \mathrm{~kg} / \mathrm{m}^{3}, \mathrm{c}_{\mathrm{p}}=1450 \mathrm{~J} /(\mathrm{kg} \mathrm{K}), \lambda=0,035 \mathrm{~W} /(\mathrm{m} \mathrm{K})[3]$ 
Initialisiert wird der Modellraum mit den Lufttemperaturen 297 K, 302 K, 307 K oder $312 \mathrm{~K}$ und das Labor mit der Lufttemperatur $292 \mathrm{~K}$. Diese Temperaturen werden in Anlehnung an die Laborversuche gewählt und führen zu den StartTemperaturdifferenzen über das Kippfenster von $5 \mathrm{~K}, 10 \mathrm{~K}, 15 \mathrm{~K}$ und $20 \mathrm{~K}$. Die Start-Konzentration des Tracergases beträgt 100 ppm im Modellraum. Auf die Modellierung eines Heizkörpers wird bei den Simulationen verzichtet.

Die Iterationen erfolgen mit konstanten Zeitschritten, wobei im Laufe der Simulation der Startzeitschritt von 0,1 s sukzessive auf ca. $1 \mathrm{~s}$ erhöht wird. Infolge der sehr kurzen Zeitschritte und der großen Anzahl an Gitterpunkten sind für die Berechnung von ca. 10 min Echtzeit ungefähr sieben Tage Simulationszeit zu veranschlagen. Der Großteil der durchgeführten Simulationen erfolgt mit einem PC Athlon 1400 und 1 GB RAM. Einige Simulationen werden mit einem PC Athlon 1800 und 0,5 GB RAM durchgeführt.

Neben der Simulation der Zuluftvolumenströme werden auch z.T. Lufttemperaturen an Raumpositionen berechnet, für die im Rahmen der messtechnischen Untersuchungen Messwerte ermittelt werden. Die simulierten und messtechnisch bestimmten Werte werden gegenübergestellt.

Um einen Einblick in die Luftbewegungen im Raum während der Lüftungszeit zu erlangen, werden exemplarisch für einen Fall die simulierten Strömungspfade der ein- und ausströmenden Luft aufgezeigt. 


\section{Vergleich Messung - CFD-Simulation}

\subsection{Gegenüberstellung der Randbedingungen}

Für die Simulation werden das Labor und der Modellraum jeweils mit einer konstanten Temperatur initiiert. Die Initiierung des Modellraumes mit Tracergas erfolgt mit einer exakten Konzentration. Es liegt eine ideale Durchmischung in ruhender Luft vor. Zum Startzeitpunkt ist das Fenster geöffnet. Die Simulationsergebnisse der einzelnen Parameter werden pro Rechenschritt als volumen- bzw. flächengemittelte Werte abgespeichert. Der Einfluss von Strahlung wird nicht berücksichtigt. Die Geometrie des Modells entspricht im Detail nicht der Realität. Die Rahmentiefe des Blendrahmens und des Kippflügels sind nicht abgebildet.

In der Realität wird im Modellraum vor und nach Beendigung der Messung mit zwei Ventilatoren eine vollständige Durchmischung von Tracergas und Raumluft angenähert. Als Folge der aktiven Durchmischung befindet sich zur Startzeit eine leichte Luftbewegung im Modellraum. Das Fenster ist während der Mischzeit geschlossen. Zum Startzeitpunkt der Messung werden die Ventilatoren abgestellt und das Fenster von außen geöffnet. Die Konzentration des Tracergases wird an vier Stellen im Modellraum bzw. Labor entnommen (Bild 8) und daraus eine mittlere Konzentration bestimmt. Lokale Konzentrationsschwankungen können nicht ausgeschlossen werden. Die Messzeiterfassung erfolgt durch einen Fensteröffnungskontakt.

\subsection{Gegenüberstellung der Ergebnisse}

\subsubsection{Zuluftvolumenströme}

In Bild 26 sind die Zuluftvolumenströme aus der rechnerischen und der messtechnischen Tracergas-Messung in Abhängigkeit von der anliegenden Temperaturdifferenz für die Kippweiten $6 \mathrm{~cm}$ und $10 \mathrm{~cm}$ und der Variante 'ohne Laibung / ohne Heizung' aufgetragen. Es zeigt sich, dass für beide Kippweiten mit der CFDSimulation ein größerer Volumenstrom als durch die Messungen bestimmt wird. Bei einer Temperaturdifferenz von $5 \mathrm{~K}$ beträgt die Differenz ca. $24 \%$ bei beiden Kippweiten. Für die Temperaturdifferenz von $20 \mathrm{~K}$ liegen die Ergebnisse von Messung und Rechnung für beide Kippweiten nur noch ca. $8 \%$ auseinander. Im Mittel beträgt die Differenz rund $13 \%$.

Der Grund für die auftretenden Abweichungen liegt vermutlich darin, dass der Fensterrahmen nicht detailliert modelliert ist. Die in der Realität vorhandene Tiefe des Rahmens sowie der Überschneidungsbereich vom senkrechten Fensterrahmen und des Kippflügelrahmens finden im Modell keine Berücksichtigung, d.h. einerseits weist die Geometrie der Strömungsöffnung einen geringeren Strömungswiderstand als die reale Öffnungsgeometrie auf und andererseits ist die lichte Öffnungsfläche des Kippfensters in der Simulation um ca. $12 \%$ größer als die reale Fläche (Abschnitt Flaeche). Trotz der Vereinfachungen in der Modellierung der Geometrie sind 
die Übereinstimmungen der berechneten und gemessenen Ergebnisse als sehr gut zu erachten.

Bei der Modellierung von einer entsprechenden Rahmentiefe bzw. von einer Laibung treten Schwierigkeiten bei der Gittergenerierung auf. Die Notwendigkeit, lokal ein sehr feines Gitter im Bereich des Fensterflügels zu erzeugen, welches mit einem weniger feinen Gitter im Modellraum korrespondiert, führt zu Problemen.

Numerische Probleme treten bei der Simulation der Kippweite von $3 \mathrm{~cm}$ auf. Es kann keine Konvergenz erreicht werden.

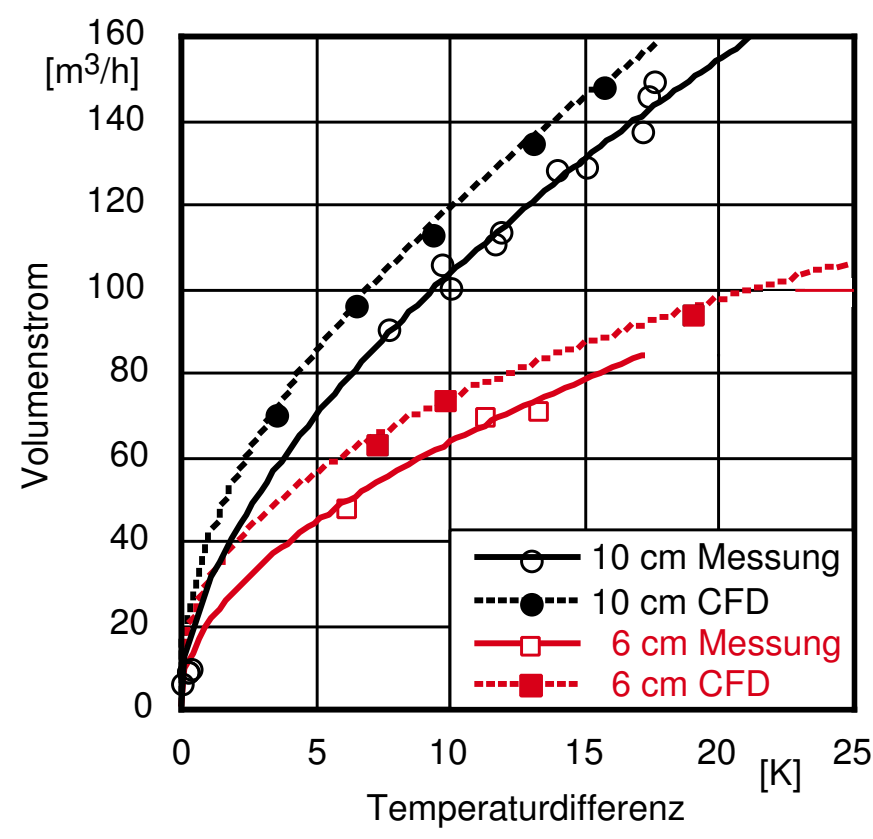

Bild 26: Vergleich der Zuluftvolumenströme der 'rechnerischen' und der messtechnischen Tracergas-Messung (Kippweite $6 \mathrm{~cm}$ und $10 \mathrm{~cm}$, ohne Laibung, ohne Heizung).

Die aus den Massenströmen berechneten Volumenströme über das Rechteck - Abluftvolumenstrom - und die beiden Dreiecke - Zuluftvolumenstrom - sind in Bild 27 für die Kippweite von $10 \mathrm{~cm}$ in Abhängigkeit von der Zeit aufgetragen. Die Kurve des Volumenstroms, der durch die Dreiecke strömt, stellt die Summe aus den Volumenströmen durch beide Dreiecke dar. Die mittlere Temperaturdifferenz beträgt $16 \mathrm{~K}$. Die Wandtemperaturen sind konstant bzw. frei gehalten.

Die entsprechend berechneten Zuluftvolumenströme aus der simulierten TracergasMessung sind ebenfalls in Bild 27 dargestellt. Bei konstant gehaltenen Wandtemperaturen ergibt sich nach ca. $250 \mathrm{~s}$ ein konstanter Volumenstrom. Im Fall der variabel gehaltenen Wandtemperaturen stellt sich ein variabler Volumenstrom ein, der nach rund $100 \mathrm{~s}$ ein Maximum erreicht.

Die jeweils maximalen Volumenströme weisen denselben Wert bei fast identischer Temperaturdifferenz - $\approx 16 \mathrm{~K}$ - auf. Dieser Wert wird für den Vergleich mit den Messwerten herangezogen, d.h. mit beiden Varianten kann der Zuluftvolumenstrom über eine rechnerische Tracergas-Messung bestimmt werden. 
Vergleicht man den aus dem Massenstrom bzw. aus der Tracergas-Messung ermittelten Zuluftvolumenstrom miteinander, ist zu erkennen, dass der aus dem Massenstrom bestimmte Zuluftvolumenstrom rund $40 \%$ kleiner ist als der über die berechnete Tracergas-Messung ermittelte Volumenstrom. Die Ursache liegt darin, dass an den seitlichen Öffnungen eine bidirektionale Strömung anliegt, der Massenstrom jedoch flächengemittelt berechnet wird und sich damit ein Teil des $\mathrm{Zu}$ - und Abluftstromes aufhebt. Eine Berechnung des Zuluftvolumenstroms aus dem Massenstrom, wenn er nicht in absolute Massenströme in beide Richtungen aufgelöst werden kann, führt zu falschen Werten.

Insgesamt zeigt sich, dass die zeitlichen Verläufe der verschieden berechneten Volumenströme unterschiedlich verlaufen. Während die aus dem Massenstrom berechneten Volumenströme zu Beginn der Lüftung einen maximalen Volumenstrom aufweisen, der im Laufe der Lüftungszeit abnimmt, erreichen die aus der rechnerischen Tracergas-Messung berechneten Volumenströme erst nach einer bestimmten Zeit einen maximalen Wert.

Dieses Verhalten wird darauf zurückgeführt, dass sich sofort nach dem Start der Simulationsrechnung ein Massenstrom einstellt, jedoch z.T. eine Kurzschlussströmung entsteht. Ein Teil der einströmenden Luft strömt direkt wieder nach außen, so dass die Konzentration im Raum nur im geringen Maße beeinflusst wird. Erst wenn ein größeres Volumen des Raumes an dem Luftwechsel beteiligt ist, erfolgt eine Reduktion der Konzentration und damit ein über die Tracergas-Technik bestimmbarer Volumenstrom.

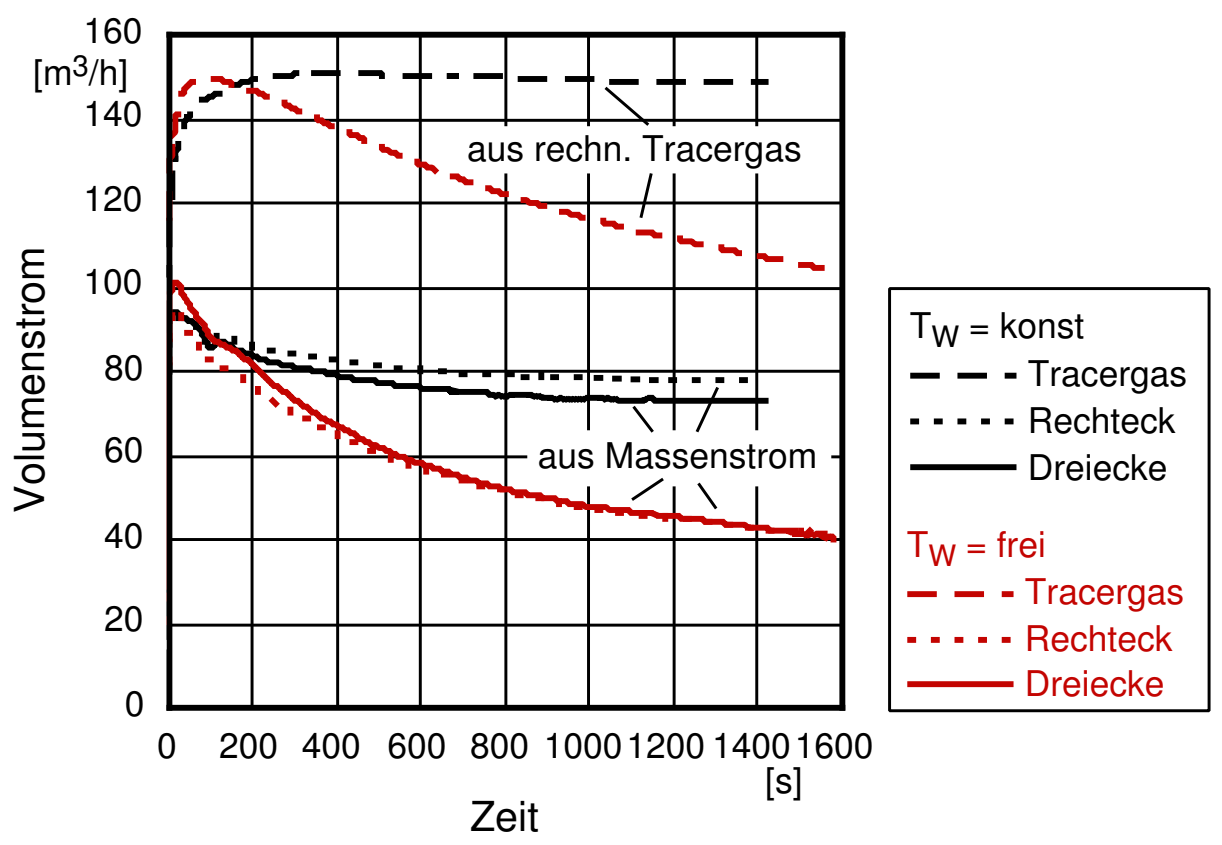

Bild 27: Volumenströme bestimmt aus dem Massenstrom und 'rechnerischen' Tracergas-Messung (Kippweite $10 \mathrm{~cm}$, ohne Laibung, ohne Heizung). 


\subsubsection{Temperaturen}

In Bild 28 ist der simulierte zeitliche Lufttemperaturverlauf an den Positionen C/D/E, H/I/J und M/N/O, analog der gemessenen Positionen, dargestellt. Für die Simulation sind die Temperaturen der Wände konstant gehalten. Initiiert ist der Modellraum mit $39{ }^{\circ} \mathrm{Cund}$ das Labor mit $19{ }^{\circ} \mathrm{C}$. Die Kippweite beträgt $10 \mathrm{~cm}$ (Variante 'ohne Laibung / ohne Heizung').

Auffällig ist, dass die Temperaturverläufe und -schichtungen in allen drei Bildern sehr ähnlich sind. Deutlich sind Gruppierungen der Temperaturen in Abhängigkeit von der Höhe zu erkennen. Dies deutet stark auf eine Bildung von horizontalen Temperaturschichten hin. In der untersten Schicht - ca. $20 \mathrm{~cm}$ vom Boden entfernt - ist eine Temperaturerhöhung von ca. $1 \mathrm{~K}$ vom fensternahen zum fensterfernen Bereich zu verzeichnen.

Der Vergleich mit den Messwerten aus Abschnitt TempImModellraum1 zeigt, dass die berechneten Temperaturverläufe den gemessenen Temperaturverläufen für die Variante 'ohne Laibung / ohne Heizung' z.T. ähnlich sind. Der exponentielle Temperaturabfall in den ca. ersten 10 min sowie die annähernd konstante Temperaturverteilung über die Raumbreite stimmen überein. Die tiefste Temperatur tritt in beiden Temperaturverläufen bodennah im Bereich des Fensters auf.

Deutliche Unterschiede zwischen den Temperaturverläufen sind zum einen in dem Temperaturabfall in den ersten $10 \mathrm{~min}$ zu verzeichnen. Die berechneten Temperaturen fallen in etwa der gleichen Zeit um 1 - $2 \mathrm{~K}$ tiefer als die gemessenen Temperaturen. Zum anderen stellt sich bei den berechneten Temperaturen nach ca. $10 \mathrm{~min}$ eine konstante Temperaturschichtung ein. Dieses Verhalten ist bei den gemessenen Temperaturen nur in Verbindung mit der Heizung festzustellen.

In Bild 29 ist der Lufttemperaturverlauf mit variabel gehaltener Wandtemperatur dargestellt. Deutlich ist ein exponentieller Abfall der Temperaturen in Abhängigkeit von der Zeit zu erkennen. Es stellt sich kein konstanter Zustand ein, somit entsprechen die berechneten Temperaturverläufe qualitativ den gemessenen Temperaturverläufe von Variante 'ohne Laibung / ohne Heizung' (Abschnitt TempImModellraum1). Der Temperaturabfall in den ca. ersten $10 \mathrm{~min}$ ist bei den berechneten Verläufen jedoch deutlich größer als bei den gemessenen, d.h. die berechneten Temperaturen liegen um ca. $5 \mathrm{~K}$ niedriger als die gemessenen.

Die Diskrepanz der Temperaturen kann nur auf erhöhte Wärmeverluste über die Wände bei der Simulation zurückgeführt werden. Der Wärmeübergang wird von CFD überschätzt. Der Ansatz zur Bestimmung der thermischen Grenzschicht ist u.a. eine Funktion von der turbulenten Prandtl-Zahl $\operatorname{Pr}_{t}$ [21, 33]. Die turbulente Prandtl-Zahl stellt das Verhältnis der turbulenten Austauschgrößen von Impuls- und Wärmeaustausch da. In [33] werden turbulente Prandtl-Zahlen für Gase zwischen 0,7 und 1 angegeben. In Fluent beträgt der default-Wert $\operatorname{Pr}_{t}=0,85$ [21], d.h. es wird der Mittelwert aus dem o.g. Bereich eingesetzt.

Da die Prandtl-Zahl das Verhältnis von Impuls- zu Wärmeaustausch beschreibt, muss die Prandtl-Zahl größer werden, wenn der Wärmeaustausch reduziert werden soll, d.h. $\operatorname{Pr}_{\mathrm{t}}=0,85$ ist für diesen Fall zu klein. Nach [19] ist es legitim, die 

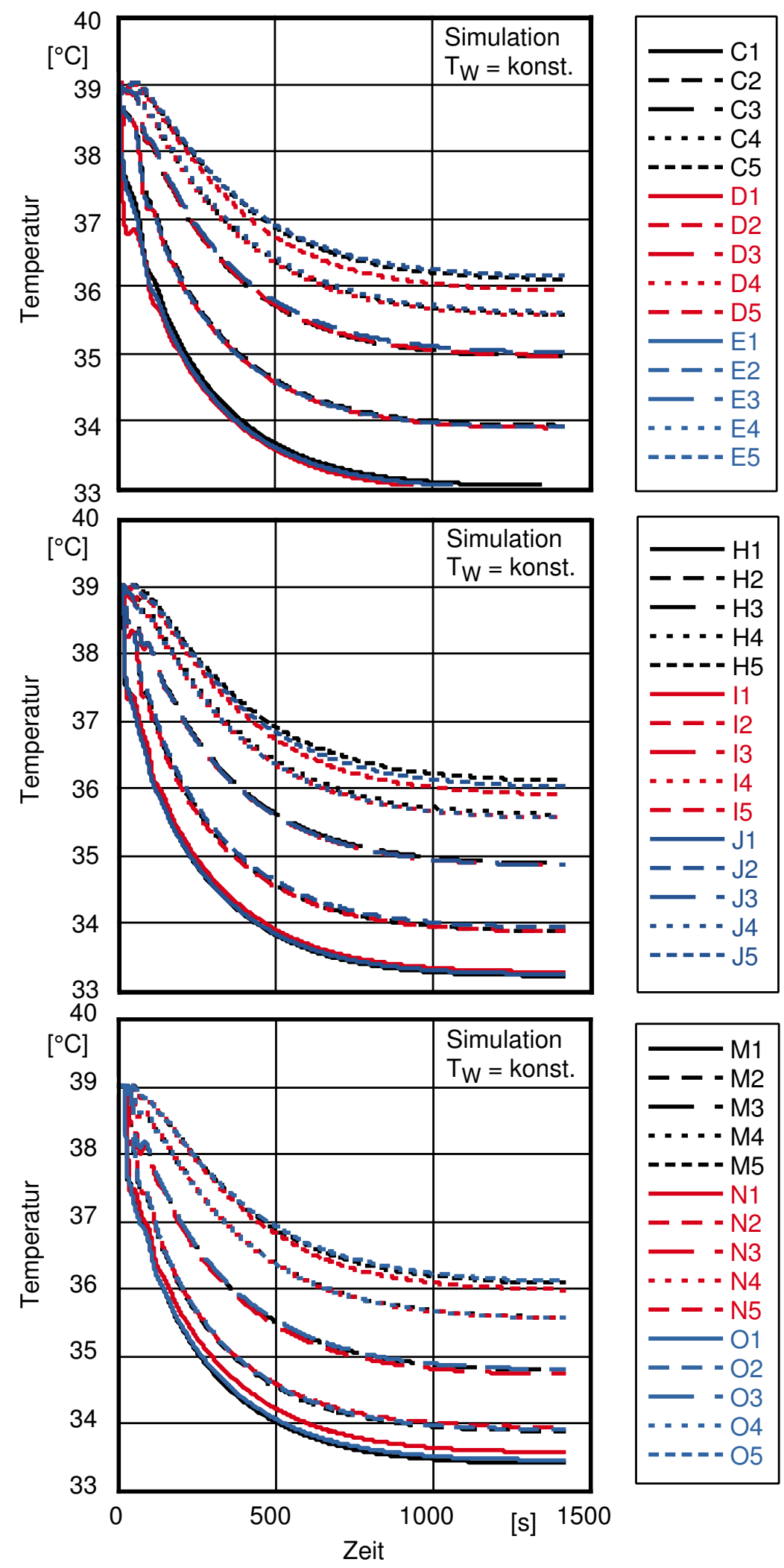

Bild 28: Darstellung der berechneten Lufttemperaturverteilung im Modellraum an den Positionen C/D/E, H/I/J und M/N/O mit konstant gehaltenen Wandtemperaturen (Kippweite $10 \mathrm{~cm}$ ). 
turbulente Prandtl-Zahl für Gase in dem Bereich zwischen 0,5 und 2 zu variieren, um damit ein simuliertes Ergebnis an Messwerte anzupassen. In den Simulationen wird der default-Wert beibehalten, so dass die Abweichungen zwischen berechneten und simulierten Lufttemperaturen auf die nicht angepasste turbulente Prandtl-Zahl zurückgeführt werden können. 


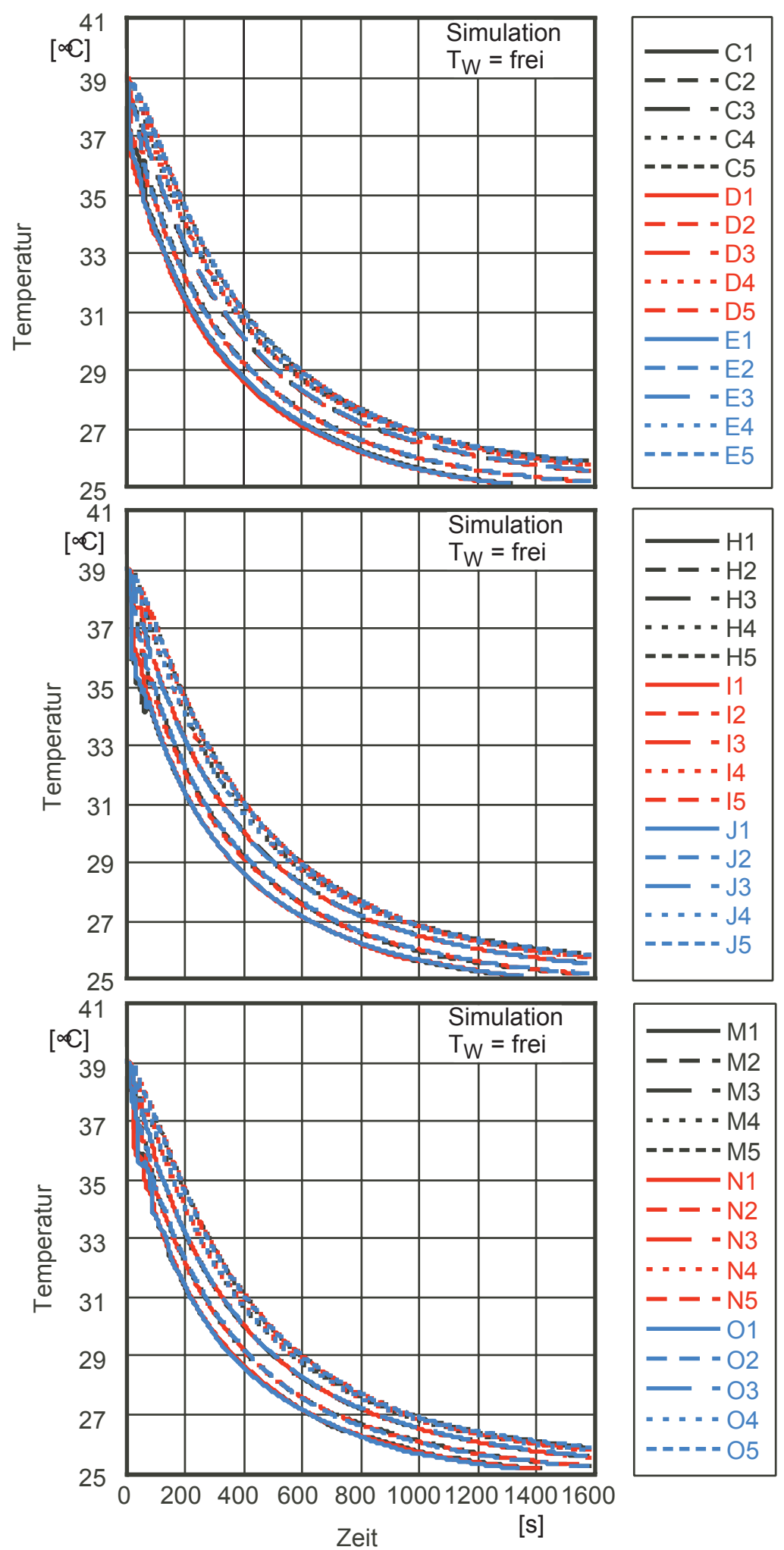

Bild 29: Darstellung der berechneten Lufttemperaturverteilung im Modellraum an den Positionen C/D/E, H/I/J und M/N/O mit variabler Wandtemperatur (Kippweite $10 \mathrm{~cm}$ ). 


\subsection{Stromlinien}

Die folgenden Bilder 30 bis 33 stellen die mit Fluent berechneten Stromlinien für die Variante ' $10 \mathrm{~cm}$ Kippweite, ohne Laibung / ohne Heizung' bei $20 \mathrm{~K}$ StartTemperaturdifferenz für verschiedene Zeitschritte - $20 \mathrm{~s}, 40 \mathrm{~s}, 59 \mathrm{~s}$ und $122 \mathrm{~s}-$ dar. Jeweils im oberen Bild sind die Stromlinien dargestellt, die die Luftbewegung ausgehend von der seitlichen Dreiecksfläche beschreiben. Anschaulich bedeutet dies, das ein Luftpaket beobachtet wird, welches in der Dreiecksfläche startet und sich entweder in den Raum oder nach außen bewegt. Da die Stromlinien, ausgehend von den beiden Dreiecksflächen, sich symmetrisch verhalten ist für die Übersichtlichkeit nur eine Seite dargestellt. Im unteren Bild sind die Stromlinien abgebildet, die in der Rechteckfläche des Kippfensters enden, d.h. es werden Luftpakete verfolgt, die im Raum starten und irgendwann in der rechteckigen Öffnungsfläche enden. Somit zeigen die Bildern 30 bis 33, welche Luftbewegungen im Raum zu erwarten sind.

Bei der Betrachtung der einströmenden Luft durch das Dreieck ist deutlich zu erkennen, dass die Luft seitlich des Fensters auf den Boden und entlang der Kante Wand / Fußboden weiter in den Raum strömt. Dabei erwärmt sich die Luft und steigt nach oben. Es bildet sich eine Walze aus, die in den rund ersten $40 \mathrm{~s}$ Lüftungsdauer immer tiefer in den Raum vordringen. Nach ca. $60 \mathrm{~s}$ Lüftungsdauer fängt die Walze an sich aufzulösen. Im weiteren kommt es zu einer Schichtenbildung, d.h. die einströmende Luft schiebt sich unter die vorhandene Luft und hebt diese als gesamte Schicht an.

Die Stromlinien zeigen, dass zu Beginn der Lüftungsdauer eine Anlaufphase stattfindet. Im vorliegenden Fall ist diese nach ein bis zwei Minuten abgeschlossen. In [14] wird ein Zeitraum zur Simulation der Massenströme zur Berechnung von Zuluftvolumenströme über ein Kippfenster von ungefähr $20 \mathrm{~s}$ angegeben, bis konstante Bedingungen zu erwarten sind. Obwohl der hierbei untersuchte Raum rund $15 \%$ und die lichte Öffnung des Fensters ca. $25 \%$ kleiner ist, als der betrachtete Modellraum und das untersuchte Fenster in dieser Arbeit, erscheint der Zeitraum als sehr kurz.

Aus dem gesamten Strömungsbild ist abzuleiten, dass in den ersten Sekunden der Öffnungsdauer mit einer starken Luftzirkulation im Bereich des Fensters zu rechnen ist. Eine Kurzschlussströmung für einen Teil der Luft kann nicht ausgeschlossen werden. Mit Hilfe der Kurzschlussströmung ist zu erklären, dass der Volumenstrom bei der Tracergasmessung erst nach einer gewissen Zeit das Maximum erreicht. Bei einer Kurzschlussströmung strömt zwar Luft über das Fenster ein und aus, jedoch ist die Konzentrationsänderung im Raum sehr gering. Erst nachdem ein größerer Teil des Raumvolumens an dem Luftwechsel beteiligt ist, erfolgt eine Reduktion der Konzentration.

Die dargestellten Stromlinien sind mit der Randbedingung 'variable Wandtemperaturen' erzeugt. Derselbe Verlauf kann mit der Bedingung 'konstante Wandtemperaturen' ermittelt werden. D.h. diese Randbedingung weist für die Ausbildung der Stromlinien eine untergeordnete Rolle auf. 

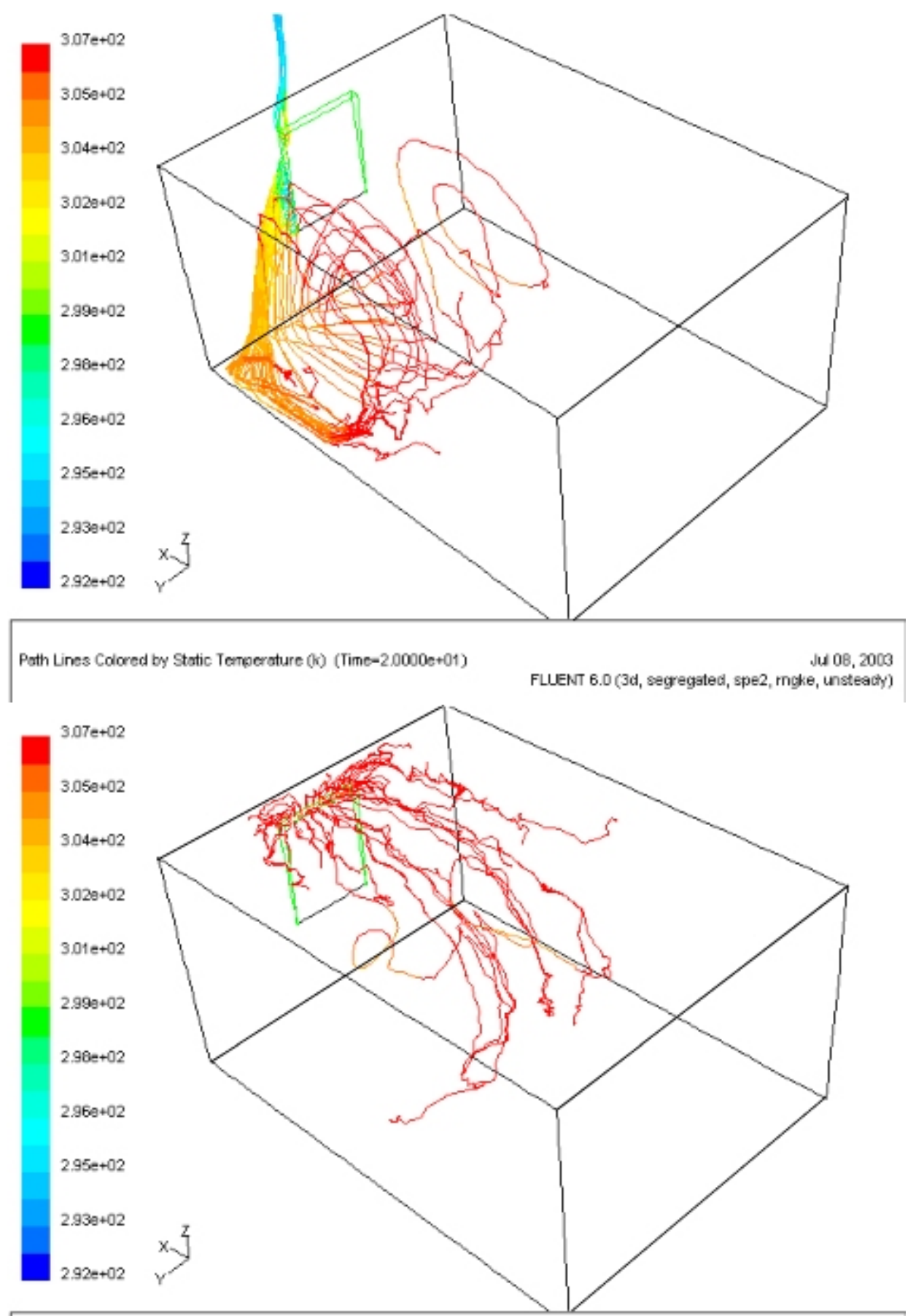

Path Lines Colored by Static Temperature (k) (Time-2.0000e+01)

Jui 08,2003 FLUENT 6.0 (3d, segregsted, spe2, mgke, unsteach)

Bild 30 Stromlinien nach $20 \mathrm{~s}$ (Kippweite $10 \mathrm{~cm}$, ohne Laibung, ohne Heizung). Die Temperaturen der Strömungspfade sind farbig dargestellt, rot: $307 \mathrm{~K}$, blau: $292 \mathrm{~K}$. 

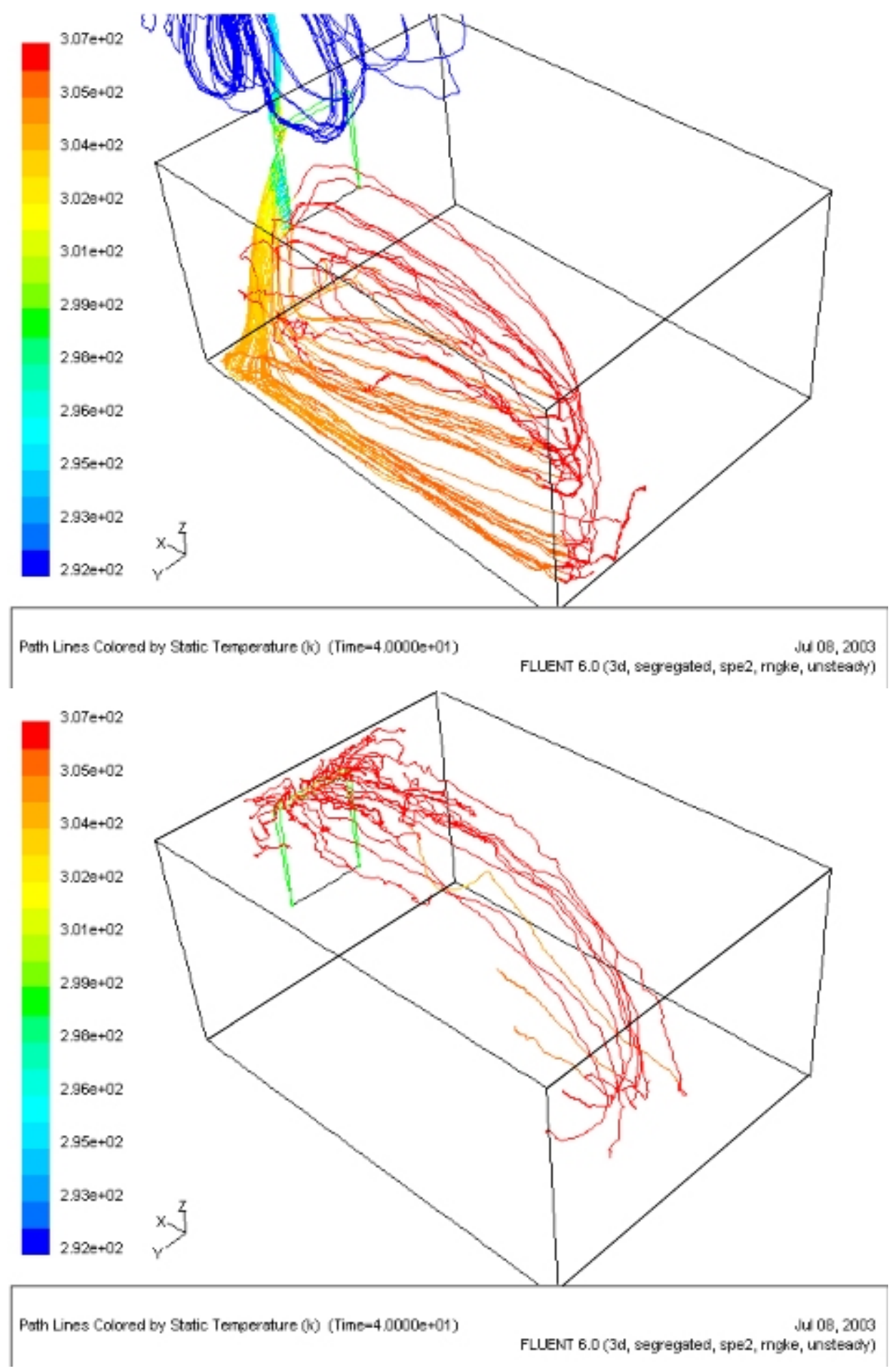

Bild 31 Stromlinien nach $40 \mathrm{~s}$ (Kippweite $10 \mathrm{~cm}$, ohne Laibung, ohne Heizung). Die Temperaturen der Strömungspfade sind farbig dargestellt, rot: $307 \mathrm{~K}$, blau: $292 \mathrm{~K}$. 

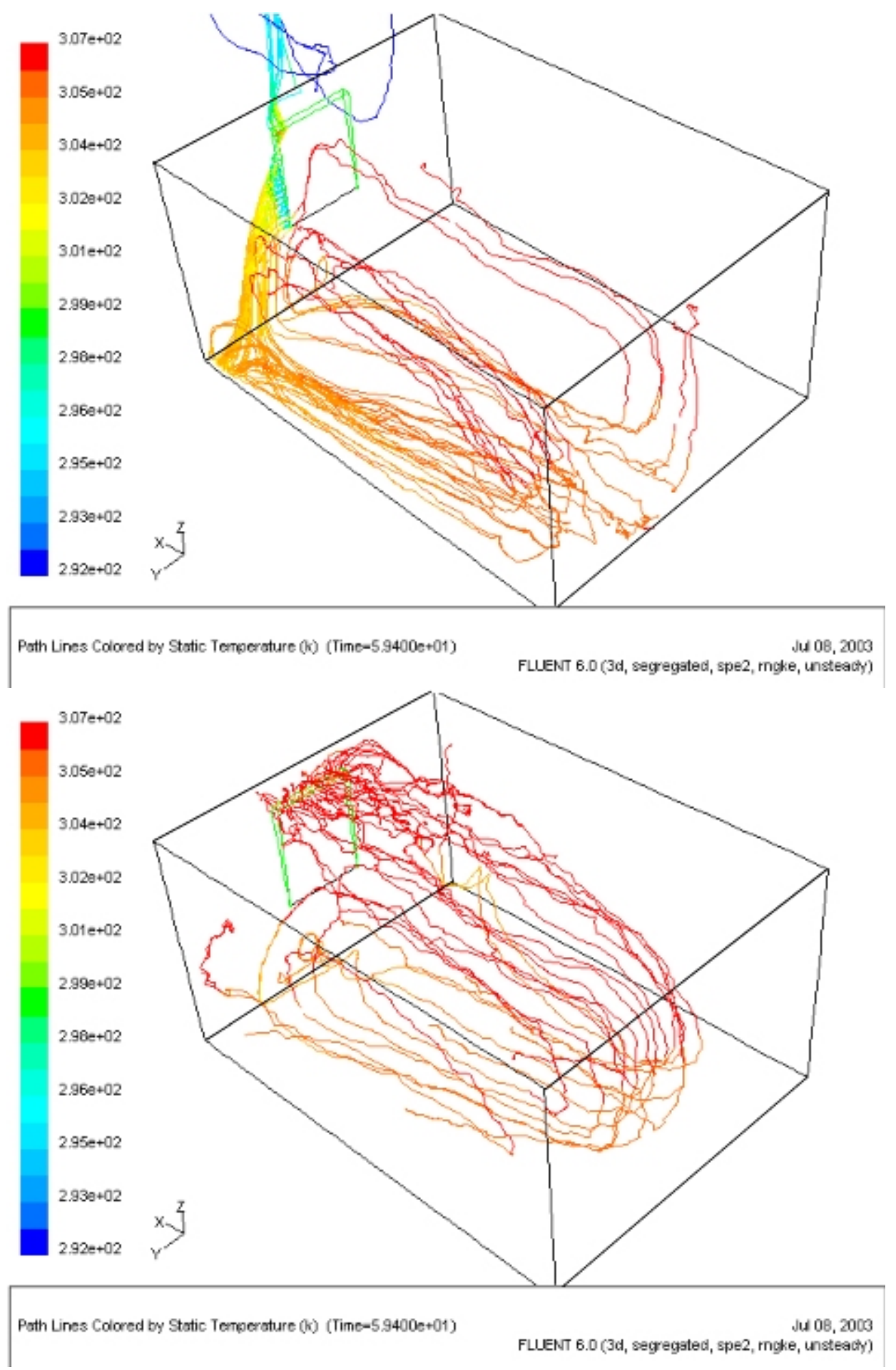

Bild 32 Stromlinien nach 59 s (Kippweite $10 \mathrm{~cm}$, ohne Laibung, ohne Heizung). Die Temperaturen der Strömungspfade sind farbig dargestellt, rot: $307 \mathrm{~K}$, blau: $292 \mathrm{~K}$. 

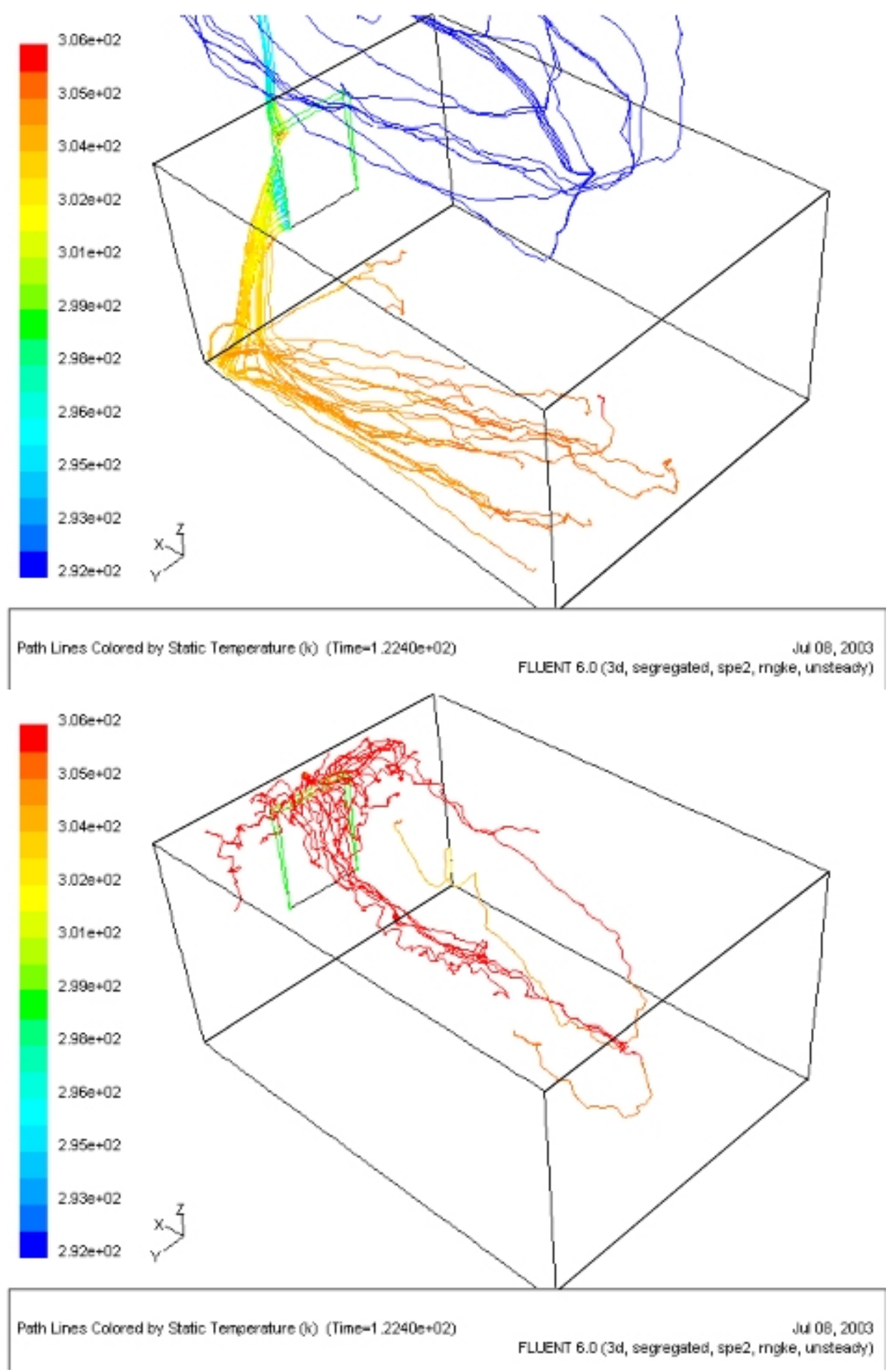

Bild 33 Stromlinien nach $122 \mathrm{~s}$ (Kippweite $10 \mathrm{~cm}$, ohne Laibung, ohne Heizung). Die Temperaturen der Strömungspfade sind farbig dargestellt, rot: $307 \mathrm{~K}$, blau: $292 \mathrm{~K}$. 


\section{Modellanpassung}

\subsection{Modellansatz}

Wie der Vergleich der gemessenen Zuluftvolumenströme mit den nach den Literaturansätzen berechneten Volumenströme zeigt, kann keine Übereinstimmung der Ergebnisse abgeleitet werden. Aus diesem Grund erfolgt eine Modellanpassung zur rechnerischen Beschreibung des thermisch induzierten Luftwechsels bei einer einseitigen Fensterlüftung mit Kippstellung. Insbesondere soll das Augenmerk auf einer verbesserten Modellierung der effektiven Öffnungsfläche gelegt werden. Die Ansätze von Daler, Weber und Maas lassen sich zu folgendem Ansatz

$$
\dot{V}_{z u}=C_{d} A_{e f f}\left(2 g H Z \frac{\Delta T}{T_{i}}\right)^{n} \quad\left[m^{3} / h\right]
$$

modifizieren. Die Reibungsverluste werden in einem Wert $-\mathrm{C}_{\mathrm{d}}-$ zusammengefasst. Die Berechnung der effektive Öffnungsfläche $A_{\text {eff }}$ variiert in Abhängigkeit von der Einbausituation (Abschnitt 8.2). Der Faktor $\mathrm{Z}$ für die Bestimmung der neutralen Höhe ist variabel gehalten.

Der Strömungsexponent $\mathrm{n}$ wird nicht konstant zu 0,5 gesetzt, sondern variabel gehalten. Der Exponent ist von der Charakteristik der Strömung abhängig und kann zwischen den Werten 0,5 und 1,0 variieren. Der Wert 0,5 beschreibt eine voll entwickelte turbulente und der Wert 1,0 eine laminare Strömung [9].

\subsection{Bestimmung der Öffnungsfläche}

\subsubsection{Allgemeines}

Für die verbesserte Beschreibung der effektiven Öffnungsfläche $\mathrm{A}_{\text {eff }}$ eines Kippfensters wird die Einbausituation des Fensters in drei Fälle unterteilt

Fall 1 Fenster ohne Laibung $(1=0)$

Fall 2 Laibungstiefe ist kleiner als die Kippweite $(0<1<\mathrm{s})$

Fall 3 Laibungstiefe ist gleich und größer als die Kippweite $(1 \geq s)$

Die Rechteckfläche lässt sich über die Breite des Fensters und der Kippweite bzw. dem minimalsten Abstand zwischen Kippflügel und Sturz bestimmen.

Die lichte Öffnungsfläche eines Dreieckes kann infolge der Überdeckung des Rahmens im unteren Teil des Fensterflügels nicht aus der gesamten Fensterhöhe abgeleitet werden (Bild 2). Im Überdeckungsbereich des Rahmens ist die Öffnungsfläche auf einen Spalt reduziert, dessen Höhe y von dem Abstand zwischen dem Blendrahmen und dem Kippflügel abhängig ist. Üblicher Weise beträgt dieser Abstand ca. 1 $\mathrm{cm}$. Die Länge x des Spaltes ist abhängig von der Fensterhöhe H, der Rahmentiefe d 
und der Kippweite s. Mit zunehmender Kippweite wird die Spaltlänge kleiner. Die reduzierte Fensterhöhe $\mathrm{h}$ berechnet sich $\mathrm{zu}$

$$
h=H\left(1+\frac{d}{s}\right)^{-1} \quad[m]
$$

Daraus lässt sich die Länge x des Spaltes zu

$$
x=H-h \quad[m]
$$

bestimmen. In Tabelle 2 sind die gemessenen und berechneten reduzierten Höhen $\mathrm{h}$ von verschiedenen Kippfenstern gegenübergestellt. Es ergibt sich nur eine geringe prozentuale Abweichung, so dass der Ansatz zur Bestimmung der reduzierten Höhe nach Gleichung 27 herangezogen werden kann.

In [59] wird die reduzierte Fensterhöhe zu

$$
h=\frac{d}{\tan (\beta)} \quad[m]
$$

bestimmt. Die Gleichungen 27 und 29 liefern das gleiche Ergebnis. Für die Praxis ist G1. 27 jedoch geeigneter, da zum einen alle Parameter am Fenster direkt gemessen werden können und zum anderen keine Verwechslung mit dem Öffnungswinkel auftritt. An einem Kippfenster können zwei verschiedene Öffnungswinkel bestimmt werden (Bild 34). Die Ansätzen von Daler, Weber und Maas verwenden Winkel $\varphi$. Dieser Winkel wird ohne Berücksichtigung einer Rahmentiefe bestimmt. In [59] wird die Rahmentiefe mit berücksichtigt, so dass hier Winkel $\beta$ bestimmt werden muss. Bei üblichen Rahmentiefen von 4,5 bis $5 \mathrm{~cm}$ ist Winkel $\varphi$ rund $25 \%$ kleiner als Winkel $\beta$.
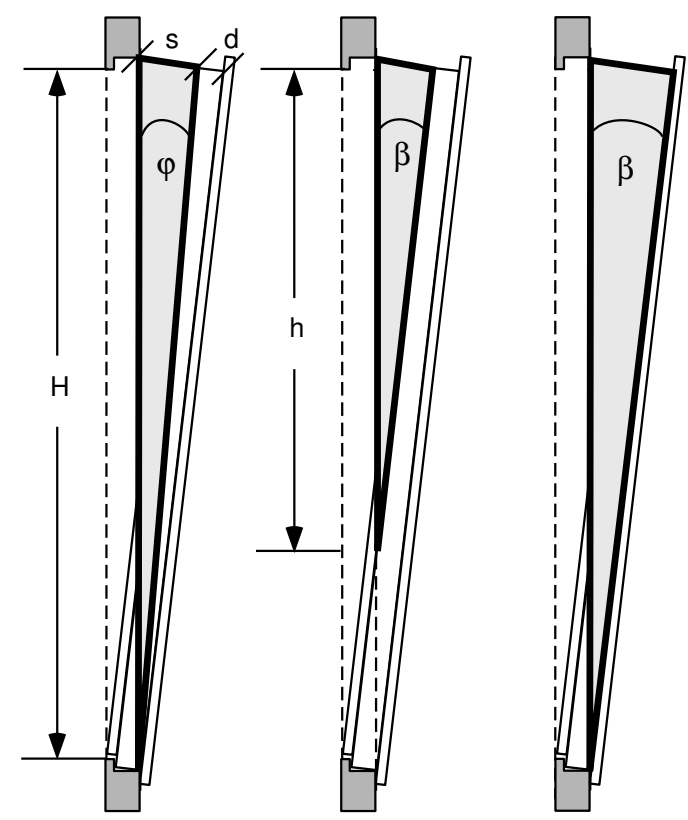

Bild 34: Verschiedene Öffnungswinkel mit / ohne Berücksichtigung der Rahmentiefe d.. 
Die Öffnungsfläche eines Standard-Kippfensters setzt sich aus einem Rechteck, zwei Dreiecken und zwei Rahmenüberschneidungen zusammen. Die gesamte Öffnungsfläche des Kippfensters berechnet sich damit zu

$$
A_{\text {ges }}=s(B+h)+2 x y \quad\left[m^{2}\right]
$$

wobei der erste Term das Rechteck und die lichten Dreiecke beschreibt und der zweite Term die Rahmenüberschneidung im unteren Bereich des Kippfensters.

Zur Vereinfachung wird die Fläche der seitlichen Dreiecke über das Produkt von Kippweite und Höhe des Dreieckes berechnet. Dies ist formal nicht ganz korrekt, da kein rechtwinkliges Dreieck vorliegt. Der Fehler in der Flächenermittlung ist jedoch vernachlässigbar klein.

Erfolgt die Berechnung der lichten Öffnungsfläche mit und ohne Berücksichtigung der Rahmenüberschneidung, dann ergeben sich für die untersuchten Kippweiten von $6 \mathrm{~cm}$ und $10 \mathrm{~cm}$ unterschiedliche Flächen. Mit Berücksichtigung der Rahmenüberschneidung vermindert sich die Fläche um rund $12 \%$ gegenüber der Varianten ohne Berücksichtigung der Rahmenüberschneidnung. Für die Kippweite von $3 \mathrm{~cm}$ wird in beiden Fällen eine gleich große Fläche bestimmt.

Der Faktor Z zur Bestimmung der neutralen Höhe wird nach Daler (Gl. 7) für alle drei Kippweiten zu 0,893 bestimmt. Nach Weber2 (Gl. 12) ergibt sich je nach Kippweite Z zu 0,687 / 0,69 / 0,694 (3 cm / $6 \mathrm{~cm} / 10 \mathrm{~cm})$. Im Mittel differieren die verschiedenen Faktoren um ca. $23 \%$. Die im Rahmen der vorliegenden Arbeit durchgeführten CFD-Simulationen führen zu $\mathrm{Z}=0,82$. Er ist für die Kippweiten von $6 \mathrm{~cm}$ bzw. $10 \mathrm{~cm}$ konstant und temperaturunabhängig. Aus eigenen Messungen wird $Z$ für die Kippweiten von $6 \mathrm{~cm}$ und $10 \mathrm{~cm}$ zu 0,83, für die Kippweite von $3 \mathrm{~cm}$ zu 0,78, bestimmt. Es kann ebenfalls keine Abhängigkeit von der Temperatur festgestellt werden. Aus Messwerten an verschiedenen Fenstern (Tabelle 3) lässt sich eine Beziehung für $\mathrm{Z}$

$$
Z=\sqrt{\frac{B-s}{H}} \quad[-]
$$

in Abhängigkeit von der Fensterbreite und -höhe sowie der Kippweite ableiten. Dieser Ansatz gilt nur für Kippfenster, deren Maße in etwa dem untersuchten Fenster entsprechen $(\mathrm{H}>\mathrm{B}$ und $\mathrm{H} \approx \mathrm{B})$. 
Tabelle 2: Vergleich der gemessenen und nach Gl. 27 berechneten reduzierten Höhe h an verschiedenen Kippfenstern.

\begin{tabular}{|c|c|c|c|c|c|}
\hline $\mathrm{H} \times \mathrm{B}$ & $\mathrm{d}$ & $\mathrm{s}$ & $\mathrm{h}_{\text {gem }}$ & $\mathrm{h}_{\text {ber }}$ & Abweichung \\
\hline$[\mathrm{m} \times \mathrm{m}]$ & {$[\mathrm{m}]$} & {$[\mathrm{m}]$} & {$[-]$} & {$[-]$} & {$[\%]$} \\
\hline \hline $1,23 \times 0,94$ & 0,045 & 0,10 & 0,865 & 0,848 & $-2,0$ \\
\hline $1,23 \times 0,94$ & 0,045 & 0,06 & 0,730 & 0,703 & $-3,8$ \\
\hline $1,23 \times 0,94$ & 0,045 & 0,03 & 0,485 & 0,492 & $+1,4$ \\
\hline $1,18 \times 0,79$ & 0,062 & 0,07 & 0,62 & 0,63 & $+1,6$ \\
\hline $1,18 \times 0,88$ & 0,062 & 0,07 & 0,62 & 0,63 & $+1,6$ \\
\hline $1,43 \times 0,88$ & 0,048 & 0,13 & 1,05 & 1,08 & $+2,8$ \\
\hline
\end{tabular}

Tabelle 3: Vergleich der gemessenen und nach Gl. 31 berechneten Faktoren $\mathrm{Z}$ zur Bestimmung der neutralen Höhe an verschiedenen Kippfenstern.

\begin{tabular}{|c|c|c|c|c|}
\hline $\mathrm{H} \times \mathrm{B}$ & $\mathrm{s}$ & $\mathrm{Z}_{\text {gem }}$ & $\mathrm{Z}_{\text {ber }}$ & Abweichung \\
\hline$[\mathrm{m} \times \mathrm{m}]$ & {$[\mathrm{m}]$} & {$[-]$} & {$[-]$} & {$[\%]$} \\
\hline \hline $1,23 \times 0,94$ & 0,10 & 0,83 & 0,82 & $-1,2$ \\
\hline $1,23 \times 0,94$ & 0,06 & 0,83 & 0,85 & $+2,3$ \\
\hline $1,23 \times 0,94$ & 0,03 & 0,78 & 0,86 & $+9,3$ \\
\hline $1,18 \times 0,79$ & 0,07 & 0,81 & 0,78 & $-3,8$ \\
\hline $1,18 \times 0,88$ & 0,07 & 0,81 & 0,83 & $+2,4$ \\
\hline $1,43 \times 0,88$ & 0,13 & 0,69 & 0,72 & $+4,2$ \\
\hline
\end{tabular}




\subsubsection{Fall 1: ohne Laibung}

Infolge der bidirektionalen Strömung in dem oberen Bereich der Dreiecke kann die reduzierte Höhe h nicht über die gesamte Länge zur Bestimmung der lichten Fläche für den Zuluftvolumenstrom angesetzt werden. Sie muss um die Höhe H(1-Z) reduziert werden. Die maximale Fläche für den Zuluftvolumenstrom eines Kippfensters ohne Laibung ergibt sich zu

$$
A_{\text {max }}=s Z[h-H(1-Z)]+2 x y \quad\left[m^{2}\right]
$$

Der erste Term beschreibt die Fläche der beiden reduzierten Dreiecke und der zweite Term die Fläche im Bereich der Rahmenüberdeckung.

Das Verhältnis von der maximalen Fläche $\mathrm{A}_{\max }$, die für den Zuluftvolumenstrom genutzt werden kann, zur gesamten Öffnungsfläche des Kippfensters $A_{\text {ges }}$ beträgt ca. $34 \%$ für die Kippweiten $6 \mathrm{~cm}$ und $10 \mathrm{~cm}$. Für die Kippweite $3 \mathrm{~cm}$ ergibt sich das Verhältnis zu rund $42 \%$ (mit $\mathrm{Z}_{\text {gem }}$ ).

In [59] wird dieses Verhältnis durch den Einschnürungsfaktor $\mu$ ausgedrückt und an einem Kippfenster mit den lichten Öffnungsmassen von 2,1 x 0,67 m ( $\mathrm{H}$ x B) und den Kippweiten von 9,5 cm bzw. $19 \mathrm{~cm}$ zu $\mu=0,33$ abgeschätzt. Für übliche Öffnungsweiten kann also davon ausgegangen werden, das rund ein Drittel der gesamten Öffnungsfläche eines Kippfensters für das Einströmen der Zuluft zur Verfügung steht.

\subsubsection{Fall 2: Laibungstiefe kleiner als die Kippweite}

Ist die raumseitige Laibungstiefe kleiner als die Kippweite, wird die lichte Kippöffnung zum Teil durch die Laibung begrenzt. Die Öffnungsfläche berechnet sich $\mathrm{zu}$

$$
A_{s}=[B a+p(s-l)+2 m q+2 x y] \frac{A_{\max }}{A_{\text {ges }}} \quad\left[m^{2}\right]
$$

unter der Annahme, dass das Flächenverhältnis von der Öffnungsfläche, die für den Zuluftvolumenstrom zur Verfügung steht, zur Gesamtfläche konstant bleibt. Der erste Term beschreibt die Fläche zwischen Kippflügel und Laibungssturz, der zweite Term den seitlichen Bereich, der nicht von der Laibung überdeckt wird, der dritte Term den Bereich des Fensters, der seitlich von der Laibung begrenzt wird, und der vierte Term den Bereich der Rahmenüberschneidung (Bild 35).

Der Abstand a zwischen Kippflügel und Laibungssturz lässt sich aus

$$
a=\sqrt{(s-l)^{2}+j^{2}} \quad[m]
$$

ermitteln. Die Höhe des Bereiches p, der von der Laibung nicht überdeckt wird, ergibt sich zu

$$
p=\frac{(s-l)}{s} h \quad[m]
$$


und die Höhe q zwischen Kipprahmen und seitlicher Laibung wird durch

$$
q=h-p \quad[m]
$$

bestimmt. Alle anderen Maße können vor Ort mit einem Metermaß ermittelt werden.

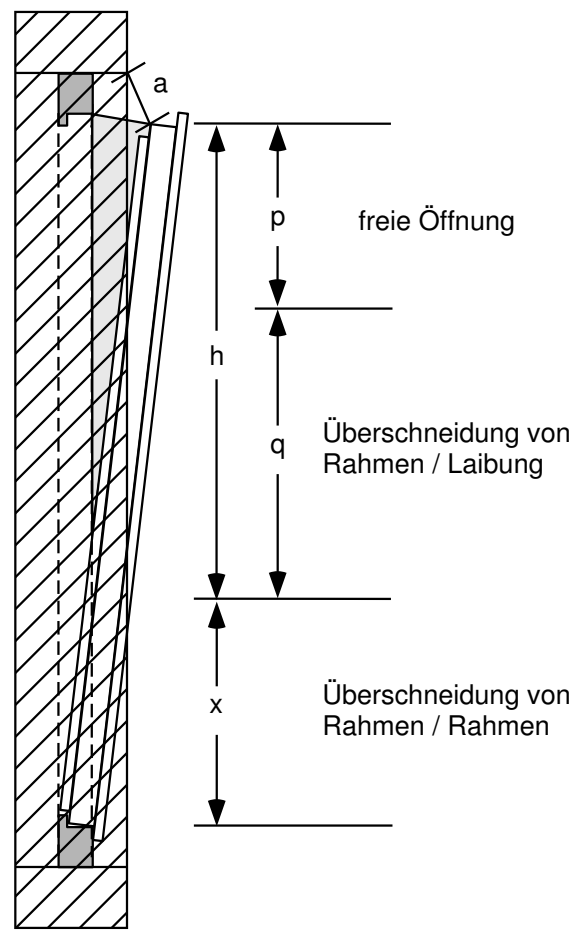

Bild 35: Verschiedene Bereiche der Dreiecke, die sich infolge einer Laibung ergeben.

\subsubsection{Fall 3: Laibungstiefe größer als die Kippweite}

Ist die Laibungstiefe größer als die Kippweite, müsste dies strenggenommen zu zwei verschiedenen Öffnungsflächen führen. Eine Öffnungsfläche ergibt sich aus der Laibungstiefe, wenn diese größer als die Summe aus Kippweite und Rahmentiefe ist. Eine andere Öffnungsfläche ergibt sich für den Fall, dass die Laibungstiefe zwar größer als die Kippweite aber kleiner als die Summe aus Kippweite und Rahmentiefe ist. Wie die Messergebnisse jedoch für die Kippweite von $10 \mathrm{~cm}$ und den Laibungstiefen von $10 \mathrm{~cm}$ und $20 \mathrm{~cm}$ zeigen, führt dies nur zu einem sehr geringen Unterschied in den bestimmten Volumenströmen. Aus diesem Grund müssen diese Fälle nicht unterschieden werden.

Ist eine raumseitige Laibung vorhanden, die größer als die Kippweite ist, muss in diesem Fall die Fläche zwischen Flügel und Laibung bzw. Sturz in Ansatz gebracht werden. Sie berechnet sich zu

$$
A_{\min }=(B j+2[h m+x y]) \frac{A_{\max }}{A_{g e s}} \quad\left[m^{2}\right]
$$


Tabelle 4: Zusammenstellung der Discharge-Koeffizienten $C_{d}$ und Strömungsexponenten $\mathrm{n}$, die sich aus den Messwerten ergeben (mit $Z_{\text {gem }}$ ).

\begin{tabular}{|c|c|c|c|c|c|c|c|c|}
\hline & \multicolumn{4}{|c|}{ ohne Laibung } & \multicolumn{4}{|c|}{ mit Laibung } \\
\hline & \multicolumn{2}{|c|}{ ohne H } & \multicolumn{2}{|c|}{ mit $\mathrm{H}$} & \multicolumn{2}{|c|}{ ohne H } & \multicolumn{2}{|c|}{ mit $\mathrm{H}$} \\
\hline$[-]$ & $\mathrm{C}_{\mathrm{d}}$ & $\mathrm{n}$ & $\overline{C_{d}}$ & $\mathrm{n}$ & $\mathrm{C}_{\mathrm{d}}$ & $\mathrm{n}$ & $\mathrm{C}_{\mathrm{d}}$ & $\mathrm{n}$ \\
\hline \multicolumn{9}{|c|}{ Messung, $\mathrm{n}=$ 'frei' } \\
\hline $10 \mathrm{~cm}$ & 0,57 & (0,62 & $\overline{0,44}$ & 0,55 & 0,65 & 0,72 & 0,48 & 0,49 \\
\hline $6 \mathrm{~cm}$ & 0,55 & 0,57 & 0,46 & 0,42 & 0,52 & 0,71 & 0,42 & 0,43 \\
\hline $3 \mathrm{~cm}$ & 0,49 & 0,72 & $\overline{0,40}$ & 0,39 & $\overline{0,44}$ & 0,55 & 0,39 & 0,27 \\
\hline $10 \mathrm{~cm}, \mathrm{r}$ & \multicolumn{2}{|c|}{0,998} & \multicolumn{2}{|c|}{1,0} & \multicolumn{2}{|c|}{0,999} & \multicolumn{2}{|c|}{0,942} \\
\hline $6 \mathrm{~cm}, \mathrm{r}$ & \multicolumn{2}{|c|}{0,982} & \multicolumn{2}{|c|}{0,982} & \multicolumn{2}{|c|}{0,984} & \multicolumn{2}{|c|}{0,995} \\
\hline $3 \mathrm{~cm}, \mathrm{r}$ & \multicolumn{2}{|c|}{0,995} & \multicolumn{2}{|c|}{0,999} & \multicolumn{2}{|c|}{0,998} & \multicolumn{2}{|c|}{0,960} \\
\hline \multicolumn{9}{|c|}{ Messung, $\mathrm{n}=0,5$} \\
\hline $10 \mathrm{~cm}$ & 0,57 & 0,50 & 0,44 & 0,50 & 0,63 & 0,50 & 0,47 & 0,50 \\
\hline $6 \mathrm{~cm}$ & 0,55 & 0,50 & 0,47 & 0,50 & 0,49 & 0,50 & 0,42 & 0,50 \\
\hline $3 \mathrm{~cm}$ & 0,45 & 0,50 & 0,41 & 0,50 & 0,44 & 0,50 & 0,41 & 0,50 \\
\hline $10 \mathrm{~cm}, \mathrm{r}$ & \multicolumn{2}{|c|}{0,996} & \multicolumn{2}{|c|}{0,999} & \multicolumn{2}{|c|}{0,991} & \multicolumn{2}{|c|}{0,942} \\
\hline $6 \mathrm{~cm}, \mathrm{r}$ & \multicolumn{2}{|c|}{0,980} & \multicolumn{2}{|c|}{0,966} & \multicolumn{2}{|c|}{0,958} & \multicolumn{2}{|c|}{0,980} \\
\hline $3 \mathrm{~cm}, \mathrm{r}$ & \multicolumn{2}{|c|}{0,953} & \multicolumn{2}{|c|}{0,998} & \multicolumn{2}{|c|}{0,945} & \multicolumn{2}{|c|}{0,544} \\
\hline \multicolumn{9}{|c|}{ CFD-Simulation } \\
\hline $10 \mathrm{~cm}$ & 0,64 & 0,53 & - & - & - & - & - & - \\
\hline $6 \mathrm{~cm}$ & 0,61 & 0,43 & - & - & - & - & - & - \\
\hline $10 \mathrm{~cm}, \mathrm{r}$ & \multicolumn{2}{|c|}{1,0} & & & & - & & \\
\hline $6 \mathrm{~cm}, \mathrm{r}$ & & 93 & & . & & - & & \\
\hline
\end{tabular}

Es wird ebenfalls davon ausgegangen, dass sich das Verhältnis von Einström- zur Gesamtfläche für Standard-Kippweiten durch die Laibung nicht verändert. $A_{\min }$ beschreibt die kleinste Öffnungsfläche für den Zuluftvolumenstrom, der sich zwischen Laibung und gekipptem Fensterflügel ergibt.

\subsection{Modellanpassung}

Der Discharge-Koeffizient $\mathrm{C}_{\mathrm{d}}$ und der Strömungsexponent $\mathrm{n}$ werden aus den Messwerten bzw. CFD-Rechnungen ermittelt und sind in Tabelle 4 zusammengefasst. Die Anpassung erfolgt zum einen mit variablen Strömungsexponenten und zum anderen mit einem festen Exponent von $\mathrm{n}=0$,5. Für $5 \mathrm{~K}<\Delta \mathrm{T}<30 \mathrm{~K}$ beträgt die Abweichung des Modellansatzes nach Gl. 26 zu der Fitfunktion durch die Messwerte mit frei angepasstem Exponent maximal $\pm 2 \%$, für $\mathrm{n}=0,5$ maximal $\pm 3 \%$ und zu den CFD-Berechnungen ebenfalls maximal $\pm 3 \%$.

Die Discharge-Koeffizienten variieren zwischen 0,39 und 0,65, wobei tendenziell die kleineren Werte im Zusammenhang mit der Heizung auftreten. Aus der Anpas- 
sung der $\mathrm{C}_{\mathrm{d}}$-Werte an die Messwerte folgt, dass der $\mathrm{C}_{\mathrm{d}}$-Wert unabhängig von der anliegenden Temperaturdifferenz ist, jedoch mit der Kippweite variiert. Der Vergleich der Discharge-Koeffizienten untereinander zeigt, dass die Koeffizienten von dem Vorhandensein einer Heizung stark beeinflusst werden. Ist eine Heizung vorhanden muss der Discharge-Koeffizient 'Heizung' gewählt werden. In diesem Fall ist der Einfluss einer Laibung auf $\mathrm{C}_{\mathrm{d}}$ von untergeordneter Bedeutung. Der Einfluss einer Laibung auf den Discharge-Koeffizienten ist jedoch zu berücksichtigen, wenn keine Beheizung erfolgt.

Auffällig ist, dass im Fall des frei angepassten Strömungsexponenten der Exponent Werte zwischen 0,27 und 0,72 annimmt. Werte kleiner 0,5 treten auf, wenn ein Heizkörper unter dem Fenster angeordnet ist, d.h. die von der Heizung erwärmte Luft beeinflusst die Strömung am Fenster derart, dass das gewählte Modell zur Anpassung an die Messpunkte in Frage gestellt werden muss.

Die Strömungsexponenten zwischen 0,50 und 0,72 deuten an, dass die Strömung durch das Fenster nicht immer voll turbulent entwickelt ist. Dies wird durch die Betrachtung der Rayleigh-Zahl (Abschnitt Kap:Grundlagen) bestätigt.

Die angegebenen Korrelationskoeffizienten $\mathrm{r}$ sind ein Maß dafür, wie gut das gewählte Modell die Messwerte abbildet. Passt das Modell exakt zu den Messwerten, wird r $=|1|$. Die Korrelationskoeffizienten resultieren aus einer Anpassung nach der Methode der geringsten Fehlerquadrate an die Messwerte mit dem Programm Kaleidagraph [54] (Anhang Anhangkorr).

In Bild 37 ist das Verhältnis der Volumenströme aus der Anpassung mit n = 0,5 zu der Anpassung mit freiem Strömungsexponenten für die drei Kippweiten dargestellt. Das graue Feld verdeutlicht den Bereich, in dem der Volumenstrom mit der Anpassung $\mathrm{n}=0,5$ maximal $10 \%$ von dem Volumenstrom aus der Anpassung mit freiem Exponenten abweicht. Für die Kippweiten von $6 \mathrm{~cm}$ und $10 \mathrm{~cm}$ ist die Abweichung für Temperaturdifferenzen größer $5 \mathrm{~K}$, bei fast allen Varianten geringer als $\pm 10 \%$. Bei der reduzierten Kippweite von $3 \mathrm{~cm}$ treten größere Abweichungen bei Temperaturdifferenzen kleiner ca. $7 \mathrm{~K}$ und größer rund $20 \mathrm{~K}$ auf.

Die Anpassung mit freiem Strömungsexponenten an die Messwerte weist für alle Varianten tendenziell bessere Korrelationen auf. Da keine Abhängigkeit der Exponenten von der Geometrie gefunden werden kann, ist eine rechnerische Bestimmung des Strömungsexponenten nicht abzuleiten. Aus diesem Grund wird für das modifizierte Modell im weiteren $\mathrm{n}=0,5$ gesetzt.

Für die einzelnen Varianten lässt sich ein Zusammenhang der $\mathrm{C}_{\mathrm{d}}$-Werte mit der Kippweite s $(\mathrm{n}=0,5)$ herstellen (Bild 36):

$$
\begin{gathered}
C_{d}(s)=0,930 s^{0,20} \quad[-] \quad \text { 'ohneLaibung/ohneHeizung' } \\
C_{d}(s)=0,532 s^{0,07} \quad[-] \quad \text { 'ohneLaibung/mitHeizung' } \\
C_{d}(s)=0,345+2,757 s \quad[-] \quad \text { 'mitLaibung/ohneHeizung' } \\
C_{d}(s)=0,378+0,878 s \quad[-] \quad \text { 'mitLaibung/mitHeizung' }
\end{gathered}
$$




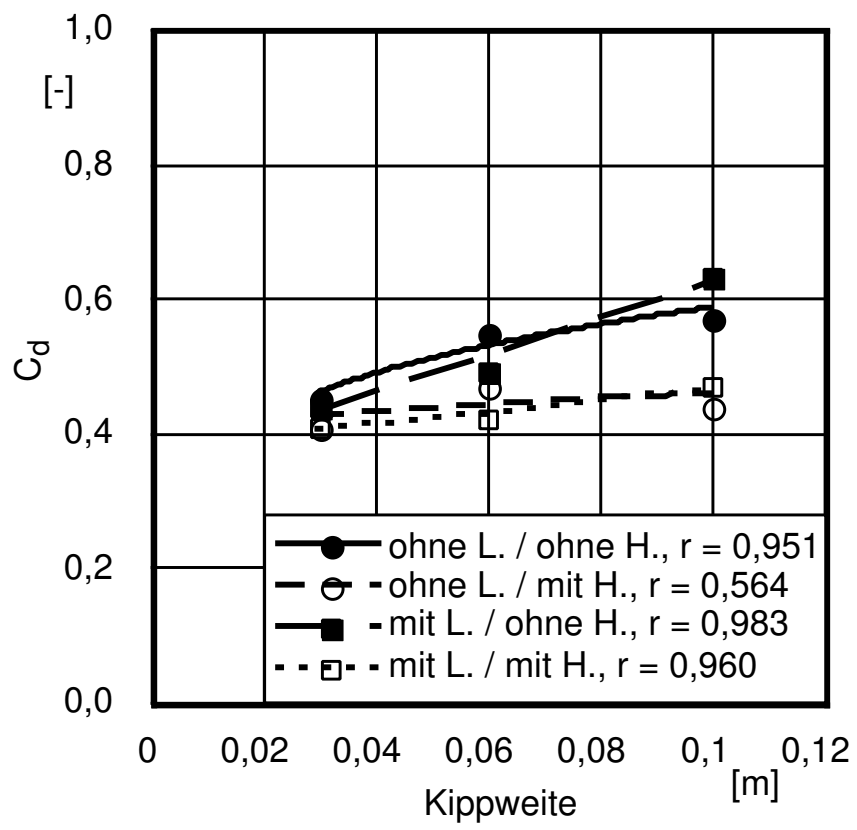

Bild 36: $C_{d}$-Werte in Abhängigkeit von der Kippweite $(n=0,5)$.

Der Vergleich Modell (Gl. 26 mit n = 0,5, Gl. 27 - 32, 37 - 41) / Messung zeigt, dass die Volumenströme eine maximale prozentuale Abweichung für die Kippweiten von $3 \mathrm{~cm}, 6 \mathrm{~cm}$ und $10 \mathrm{~cm}$ von $<25 \%,<17 \%$ bzw. $\pm 8 \%$ aufweisen.

Die mit Hilfe der CFD-Simulation gewonnenen Discharge-Koeffizienten und Strömungsexponenten für die Variante 'ohne Laibung / ohne Heizung' und den Kippweiten von $6 \mathrm{~cm}$ und $10 \mathrm{~cm}$ weichen von den aus den Messwerten gewonnenen Parameter leicht ab. Dies wird darauf zurückgeführt, dass mit den CFD-Simulationen zu große Volumenströme berechnet werden.

\subsection{Ausblick}

Zusammenfassend kann gesagt werden, dass bei dem modifizierten Ansatz nach Gl. 26 eine verbesserte Beschreibung der einzelnen Parameter durch die Geometrie erfolgt. Die effektive Öffnungsfläche und der Discharge-Koeffizient können in Abhängigkeit von der Einbausituation und die neutrale Höhe als eine Funktion von Fensterbreite und -höhe sowie der Kippweite bestimmt werden.

Die Gültigkeit des Ansatzes ist beschränkt auf Fenster mit einer ähnlichen Geometrie und Anordnung. Da nur ein Fenster untersucht wird, stellt sich nur eine Abhängigkeit des Discharge-Koeffizientens von der Kippweite ein. Es ist jedoch davon auszugehen, dass er auch eine Funktion von der Fensterbreite und -höhe ist. Dieser Zusammenhang konnte im Rahmen der Arbeit nicht untersucht werden. Hierzu sind weitergehende Untersuchungen an Standard-Fenstern mit unterschiedlicher Höhe, Breite und Kippweite durchzuführen.

Ein weiterer Schwerpunkt sollte auf die Bestimmung des Strömungsexponenten gelegt werden. Es hat sich gezeigt, dass die Anpassung mit freiem Exponenten an die 

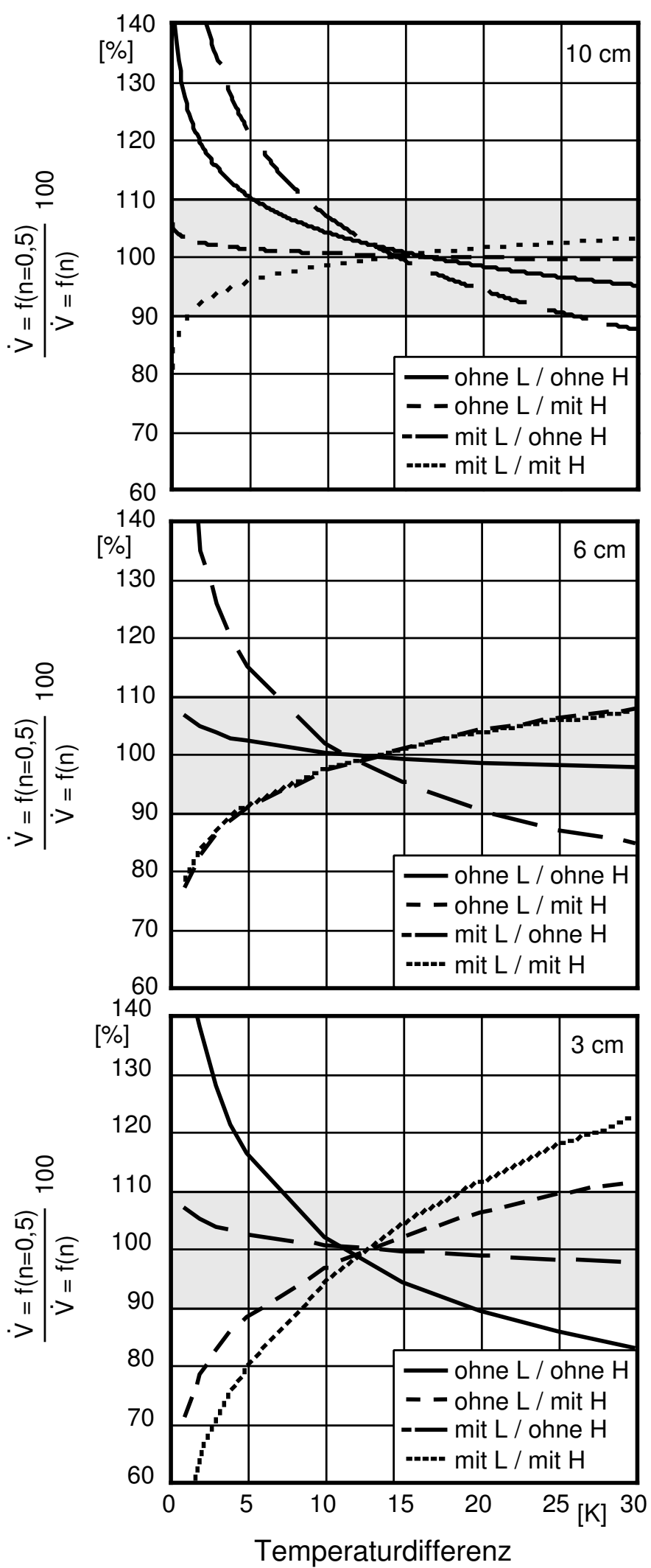

Bild 37: Verhältnis der Volumenströme aus der Anpassung von $n=0,5$ zu der Anpassung mit freiem Strömungsexponenten. 
Messwerte eine tendenziell bessere Korrelation aufweist als die Anpassung mit einem konstanten Exponenten von 0,5. Hierzu sind insbesondere Untersuchungen mit Temperaturdifferenzen kleiner als $5 \mathrm{~K}$ und größer als $25 \mathrm{~K}$ notwendig, um den temperaturabhängigen Verlauf des Zuluftvolumenstroms besser abbilden zu können.

Der Einfluss einer Heizung auf das Luftwechselpotential von Kippfenstern kann durch weitere Untersuchungen detaillierter erfasst werden. Hierbei sollten auch Heizungen mit verschiedenen Vor- und Rücklauftemperaturen und unterschiedlichen Anordnungen der Heizkörper zum Fenster, so wie z.B. Fußbodenheizungen Berücksichtigung finden. 


\section{Schlussfolgerungen}

Soll der thermisch bedingte Luftwechsel über ein bestimmtes Fenster ermittelt werden müssen, folgende Randbedingungen bekannt sein:

- Art des Fensters

- Anordnung der Drehachse

- lichte Höhe und Breite des Fensters

- Kippweite

- Tiefe des Blendrahmens

- Ist eine Laibung vorhanden? Wenn ja, müssen die Abstände zwischen Fensterrahmen und Laibung sowie die Tiefe der Laibung bekannt sein.

- Ist eine Heizung vorhanden? Wenn ja, wo ist sie im Raum positioniert? Ist sie während der Lüftungsdauer warm oder kalt?

- Welche Temperaturdifferenz soll für die Bestimmung des Luftwechsels zugrunde gelegt werden?

Die messtechnischen Untersuchungen zeigen, dass die bekannten Ansätze aus der Literatur zur Bestimmung des Zuluftvolumenstroms über Kippfenster Differenzen im Ergebnis aufweisen. Auch der Einsatz von CFD-Simulationen weist Grenzen auf. Aus diesem Grund werden für die Anwendung in der Praxis die Möglichkeiten und Grenzen der einzelnen Verfahren zusammengestellt.

Die Bestimmung des Zuluftvolumenstroms aus messtechnischen Untersuchungen ist grundsätzlich anzustreben. Der thermisch bedingte Luftwechsel über Kippfenster kann in einem entsprechend ausgerüsteten Labor ermittelt werden. Es ist möglich, das interessierende Fenster mit entsprechender Einbausituation zu untersuchen. Mit einer mobilen Tracergas-Messstation können auch Messungen vor Ort durchgeführt werden. Jedoch sind die Messergebnisse einer Vor-Ort-Messung stark von den Randbedingungen, wie u.a. der Windgeschwindigkeit und -richtung, den Innen- und AuBentemperaturen sowie der Temperaturen der benachbarten Räume und der Anbindung zu angrenzenden Räumen abhängig. Aus diesen Gründen können aus Einzelmessungen vor Ort keine allgemeingültigen Aussagen getroffen werden [37].

Der Vergleich der Messwerte mit den aus der Literatur bekannten Ansätzen zeigt, dass die Ansätze die Messwerte nicht abbilden. Die thermisch induzierten Zuluftvolumenströme werden bis auf drei Ausnahmen um $22 \%$ bis $73 \%$ geringer als die gemessenen Werte bestimmt. Der Einfluss von einer Laibung und / oder einer Heizung ist in den Ansätzen nicht berücksichtigt.

Der Einsatz von CFD-Simulationen mit Fluent 6.0 ist bedingt möglich. Zum einen kann der Luftwechsel über ein bidirektional durchströmtes Kippfenster nicht über 
Tabelle 5: Angaben zu Bereichen von Temperaturdifferenzen und Windgeschwindigkeiten, für die der Temperatureinfluss überwiegt (nach [37]).

\begin{tabular}{|c|c|}
\hline Temperaturdifferenz & Windgeschwindigkeit \\
\hline \hline $2 \mathrm{~K}-7 \mathrm{~K}$ & $<1,0 \mathrm{~m} / \mathrm{s}$ \\
\hline $18 \mathrm{~K}-21 \mathrm{~K}$ & $<2,0 \mathrm{~m} / \mathrm{s}$ \\
\hline $23 \mathrm{~K}-27 \mathrm{~K}$ & $<2,5 \mathrm{~m} / \mathrm{s}$ \\
\hline
\end{tabular}

Massenströme bestimmt werden, da die seitlichen Dreiecke nicht in $\mathrm{Zu}$ - und $\mathrm{Ab}$ strömbereiche unterteilt werden können. Zum anderen treten bei der reduzierten Kippweite von $3 \mathrm{~cm}$ numerische Probleme auf, so dass keine Ergebnisse erzielt werden können. Bei der Modellierung einer Rahmentiefe bzw. Laibung, sind Schwierigkeiten bei der Gittergenerierung festzustellen.

Eine rechnerische Tracergas-Messung mit Fluent 6.0 kann zur Bestimmung des Luftwechsel für Kippfenster mit Standard-Kippweiten ohne Laibung und ohne Heizung herangezogen werden. Die Simulationszeit sollte je nach Temperaturrandbedingung zwischen $100 \mathrm{~s}$ und $250 \mathrm{~s}$ betragen, um Anlaufeffekte auszuschliessen.

Für Kippfenster mit ähnlicher Geometrie und Einbausituation wie das in dieser Arbeit untersuchte Kippfenster bietet der Modellansatz nach Gl. eq:Moni eine verbesserte Möglichkeit, Zuluftvolumenströme zu bestimmen. Werden vor Ort die Maße der lichten Fensterhöhe und -breite, Kippweite und Rahmentiefe sowie die Laibungstiefe und die Abstände der Laibung zum Rahmen gemessen, kann die Öffnungsäche in Abhängigkeit von der Einbausituation bestimmt werden. Der Einfluss einer Laibung/Heizung wird über den entsprechenden $\mathrm{C}_{\mathrm{d}}$-Wert berücksichtigt.

Der Ansatz gilt nur für den rein thermisch induzierten Luftwechsel. Bei gleichzeitiger Einwirkung von Temperaturdifferenz und Wind kommt es zu einer Überlagerung der Effekte. Die Effekte sind weder additiv [37, 38] noch weisen sie einen linearen Zusammenhang auf [32]. Aus Untersuchungen von [37] lassen sich Bereiche ableiten, in denen der reine Temperatureinfluss überwiegt, und Bereiche, in denen der Luftwechsel maßgeblich vom Wind beeinflusst wird. In Tabelle 5 sind Grenzen der maximalen Windgeschwindigkeiten angegeben, für die bei bestimmten Temperaturdifferenzen der Temperatureinfluss überwiegt. Die ermittelten Daten gelten zwar nur für den Standort des Versuchsstandes von [37], jedoch wird deutlich, dass im Winter bei Temperaturdifferenzen von $20 \mathrm{~K}$ bis $25 \mathrm{~K}$ ein leichter Wind anstehen kann, ohne dass die Ansätze zur Bestimmung des rein thermisch induzierten Luftwechsels an Gültigkeit verlieren. 


\section{Zusammenfassung}

Das Lüften über Fenster und Türen unter natürlichen meteorologischen Bedingungen ist im Wohnbereich noch immer die häufigste Form des Lüftens. Die treibenden Kräfte für den Luftwechsel sind die Druckdifferenzen am Fenster, die durch die anliegende Temperaturdifferenz und den Wind induziert werden. Die Wirkung der Antriebskräfte wird einerseits durch die baulichen Gegebenheiten von z.B. der Fenstergröße, Öffnungsfläche, Laibungstiefe und -abstand, Anordnung des Fensters in der Fassade sowie dem Vorhandensein einer Heizung und andererseits durch den Nutzer, der z.B. Gardinen oder Rollos anbringt, beeinflusst. Der Luftwechsel resultiert aus dem komplexen Zusammenspiel von verschiedensten i.d.R. nicht beeinflussbaren Faktoren. Über den genauen Einfluss von verschiedenen Faktoren auf den Luftwechsel existieren zur Zeit keine gesicherten Erkenntnisse. Dennoch ist die Kenntnis des Luftwechsels wichtig für die Planung und Ausführung von Gebäuden in Hinblick auf das energiesparende Bauen sowie unter bauphysikalischen und hygienischen Gesichtspunkten.

Ziel dieser Arbeit ist es, den thermisch induzierten Luftwechsel über ein Kippfenster unter Berücksichtigung verschiedener Randbedingungen zu beschreiben. Die Arbeit gliedert sich in drei Teile: Im ersten Teil werden messtechnische Untersuchungen vorgestellt, im zweiten Teil CFD-Simulationen durchgeführt und im dritten Teil ein verbesserter Modellansatz zur Beschreibung des Luftwechsels aus den Messwerten abgeleitet.

Die Basis der vorliegenden Arbeit bilden messtechnische Untersuchungen an einem realen Kippfenster in einem Modellraum im Maßstab 1:1, der sich in einem großen Labor befindet. Mit Hilfe des Tracergas-Messverfahrens wird der thermisch bedingte Luftwechsel bei verschiedenen Kippstellungen, Laibungstiefen und Heizungsanordnungen untersucht. Die Ergebnisse geben Auskunft über den Einfluss der verschiedenen Randbedingungen auf den Luftwechsel.

Es wird ein handelsübliches Kippfenster ( $\mathrm{H}$ x B: 1,23 x 0,94 m) mit der StandardKippweite von $10 \mathrm{~cm}$ sowie den reduzierten Kippweiten von $3 \mathrm{~cm}$ und $6 \mathrm{~cm}$ betrachtet. Die Messergebnisse für die Zuluftvolumenströme lassen sich wie folgt zusammenfassen:

- Der Einfluss einer innenliegenden Laibung von $20 \mathrm{~cm}$ Tiefe ist insbesondere bei der Standardkippweite von $10 \mathrm{~cm}$ zu verzeichnen. Die Reduktion zu der Variante 'ohne Laibung' beträgt ca. $15 \%$.

- Für alle drei Kippweiten wird der Volumenstrom durch das Vorhandensein einer Heizung gegenüber der Variante 'ohne Laibung / ohne Heizung' um ca. $20 \%$ verringert.

- Eine Kombination von raumseitiger Laibung und Heizung vermindert das Luftwechselpotential um rund $42 \%$ bei der Standardkippweite und um ca. $25 \%$ bei den reduzierten Kippweiten.

- Eine außenseitige Laibung von $10 \mathrm{~cm}$ bzw. $20 \mathrm{~cm}$ Tiefe hat keinen Einfluss auf den Zuluftvolumenstrom. 
- Ist der Heizkörper an der dem Fenster gegenüberliegenden Wand angeordnet, kann ein größerer Volumenstrom bestimmt werden, als wenn der Heizkörper direkt unterhalb des Fensters steht. Die Erhöhung beträgt durchschnittlich 20 $\%$.

- Eine Gardine führt zu einem leicht reduzierten Volumenstrom. Die Reduktion beträgt ca. $10 \%$. Jedoch stellt die verwendete Gardine erst ab einer Temperaturdifferenz von $15 \mathrm{~K}$ einen Strömungswiderstand dar.

- Für die Variante 'ohne Laibung / ohne Heizung' weist der Volumenstrom einen annähernd linearen Zusammenhang mit der Öffnungsfläche auf. Dieser lineare Zusammenhang ist für die Variante 'mit Laibung / mit Heizung' nicht mehr gegeben.

Die Verläufe der Lufttemperatur werden an verschiedenen Stellen im Modellraum für die Varianten 'ohne Laibung / ohne Heizung', 'mit Laibung / ohne Heizung' und 'mit Laibung / mit Heizung', bestimmt. Es können folgende Aussagen abgeleitet werden:

- Die kalte Luft sammelt sich am Boden und wird durch neue einströmende Luft nach oben gehoben. Hieraus entsteht eine Temperaturschichtung im Raum, die über die Raumbreite weitgehend konstant ist.

- Über die Raumtiefe liegt kein konstantes Temperaturniveau vor. Während bei den Varianten 'ohne Heizung' die tiefste Temperatur im fensternahen Bereich am Boden zu finden ist, stellt sich die niedrigste Temperatur bei der Variante 'mit Heizung' bodennah an der dem Fenster gegenüberliegenden Wand ein. Die höchsten Temperaturen im Raum sind jeweils diagonal gegenüber zu verzeichnen.

- Die Lufttemperaturen zeigen für alle drei Varianten in den ca. ersten $10 \mathrm{~min}$ Öffnungszeit einen exponentiellen Abfall. Während bei den Varianten 'ohne Heizung' der Temperaturabfall nach rund $10 \mathrm{~min}$ in einen linearen Verlauf übergeht und über die gesamte restliche Lüftungsdauer anhält, stellen sich bei der Variante 'mit Heizung' überall relativ konstante Temperaturen ein.

- Bei der Variante 'mit Laibung / ohne Heizung' ist ein deutlicher Lenkungseffekt durch die Laibung festzustellen. Die Luft wird in Richtung Raum kanalisiert. Die bodennahen Temperaturen mittig vor dem Fenster sind niedriger als für den Fall 'ohne Laibung'.

Simuliert wird die Variante 'ohne Laibung / ohne Heizung' für die Kippweiten $6 \mathrm{~cm}$ und $10 \mathrm{~cm}$. Die Ergebnisse der mit CFD simulierten Tracergas-Messungen weisen für beide Kippweiten im Mittel rund $13 \%$ höhere Zuluftvolumenströme im Vergleich zu den Messwerten auf. Da das Fenster ohne Rahmentiefe modelliert wird, ist die Übereinstimmung mit den Messwerten als sehr gut zu erachten.

Für die Kippweite von $3 \mathrm{~cm}$ kann keine konvergente Lösung gefunden werden. Bei der Modellierung einer entsprechenden Rahmentiefe bzw. von einer Laibung treten 
Schwierigkeiten bei der Gittergenerierung auf. Zuluftvolumenströme, bestimmt aus den Massenströmen, führen zu falschen Ergebnissen. Die bidirektionale Strömung durch die seitlichen Dreiecke kann durch den flächengemittelten Massenstrom nicht erfasst werden. Der Einsatz von CFD-Simulationen mit Fluent 6.0 weist Grenzen auf.

Die aus der Simulation erhaltenen Temperaturverläufe der Raumluft decken sich nur z.T. mit den Messwerten. Rechnungen mit den Randbedingungen 'mit bzw. ohne konstant gehaltene Wandtemperaturen' führen zu unterschiedlichen Temperaturverläufen, die nur qualitativ mit den Messwerten übereinstimmen.

Der Vergleich der messtechnisch gewonnenen Zuluftvolumenströme mit den aus der Literatur bekannten Ansätzen berechneten Volumenströmen zeigt, dass die Messwerte nicht durch die Ansätze abgebildet werden können. Die thermisch induzierten Zuluftvolumenströme werden bis auf drei Ausnahmen je nach Ansatz zwischen 22 $\%$ und $73 \%$ geringer als die gemessenen Werte bestimmt. Der Einfluss von einer Laibung und / oder einer Heizung wird in den Ansätzen nicht berücksichtigt.

Die eigenen Messdaten bilden die Grundlage für die Anpassung eines Rechenmodells. Ein besonderes Augenmerk wird auf die Bestimmung der Öffnungsfläche gelegt. Werden vor Ort die Maße

- der lichten Fensterhöhe und -breite,

- der Kippweite und

- der Rahmentiefe sowie

- die Laibungstiefe und

- die Abstände der Laibung zum Blendrahmen

gemessen, kann die Öffnungsfläche in Abhängigkeit von der Einbausituation bestimmt werden. Der Einfluss einer Heizung - bei einer Anordnung unterhalb des Fensters - wird über den entsprechenden $\mathrm{C}_{\mathrm{d}}$-Wert berücksichtigt. Der Vergleich Modell/Messung zeigt, dass die Volumenströme eine maximale prozentuale Abweichung für die Kippweiten von $3 \mathrm{~cm}, 6 \mathrm{~cm}$ und $10 \mathrm{~cm}$ von $<25 \%,<17 \%$ bzw. $\pm 8 \%$ aufweisen.

Für Kippfenster mit ähnlicher Geometrie und Einbausituation wie das in dieser Arbeit untersuchte Fenster bietet der modifizierte Modellansatz eine verbesserte Möglichkeit, Zuluftvolumenströme zu bestimmen. 


\section{Literatur}

[1] DIN 1946-6:1994-09 Raumlufttechnik, Teil 6: Lüftung von Wohnungen - Anforderungen, Ausführung, Abnahme, September 1994.

[2] E DIN 4701-1:1995-08 Regeln für die Berechnung des Wärmebedarfs von Gebäuden, August 1995.

[3] DIN EN 12524:2000-07 Wärme- und feuchteschutztechnische Eigenschaften - Tabellierte Bemessungswerte, Juli 2000.

[4] DIN 4108-2:2001-01 Wärmeschutz und Energie-Einsparung in Gebäuden, März 2001.

[5] DIN EN 13829:2001-02 Wärmetechnisches Verhalten von Gebäuden - Bestimmung der Luftdurchlässigkeit von Gebäuden - Differenzdruckverfahren, Februar 2001.

[6] DIN EN ISO 12569:2001-03: Natürlicher Lüftwechsel mit Tracergasmessung, März 2001.

[7] Verordnung über energiesparenden Wärmeschutz und energiesparende Anlagentechnik bei Gebäuden (Energiesparverordnung - EnEV), November 2001.

[8] Beiblatt 1 zu DIN V 4701-10 Energetische Bewertung heiz- und raumlufttechnischer Anlagen, Teil 10: Diagramme und Planungshilfen für ausgewählte Anlayensysteme mit Standardkomponenten, Februar 2002.

[9] Allard, F. Natural ventilation in buildings. James and James Ltd, London, 1998.

[10] ASHRAE. Handbook of Fundamentals, 2001.

[11] Asimakopoulos, M. et al. Development of a control algorithm to optimize airflow rates through variable size windows. Energy and Buildings (2002), H. 34, S. 363-368.

[12] Bartak, M. et al. Integrating CFD and building simulation. Energy and Buildings (2002), H. 37, S. 865-871.

[13] Bošnjaković, F. und Knoche, K. F. Technische Thermodynamik - Teil II. Steinkopff Verlag, Darmstadt, 6. vollst. überarb. und erw. Auflage, 1997.

[14] Cadloni, M. und Ferrazzini, M. Natürlicher Luftaustausch durch Kippfenster. Diplomarbeit, ETH Zürich, CH, 1997.

[15] Cheong, K. Airflow measurements for balancing of air distribution system tracer-gas technique as an alternative? Building and Environment (2001), H. 36, S. 955-964. 
[16] Daler, H. et al. Bestandsaufnahme von Einrichtungen zur freien Lüftung im Wohnungsbau. Forschungsbericht T84-028, Bundesministerium für Forschung und Technologie, 1984.

[17] Dascalaki, E. et al. Predicting single sided natural ventilation rates in buildings. Solar Energy (1995), H. 5, S. 327-341.

[18] Dascalaki, E. et al. On the combination of air velocity and flow measurements in single sided natural ventilation configurations. Energy and Buildings (1996), H. 24, S. 155-165.

[19] Dillmann, A. Persönliche Mitteilung, Juli 2003.

[20] Feustel, H. und Smith, B. Comis 3.0 - User's Guide, 1997.

[21] Fluent Inc., Centerra Resource Park, 10 Cavendish Court, Lebanon. FLUENT 6.0 - User's Guide, November 2001.

[22] Fracastoro, G., Mutani, G. und Perino, M. Experimental and theoretical analysis of natural ventilation by windows opening. Energy and Buildings (2002), H. 34, S. 817-827.

[23] Gehle, M. Aufbau und Inbetriebnahme eines Laborprüfstandes "Raum im Raum”. Diplomarbeit, Universität Kassel, Fachgebiet Bauphysik, 2002.

[24] Geißler, K. J. Meßergebnisse zur Luftdichtheit von Gebäuden und theoretische Ermittlung der in der Praxis erreichbaren Meßgenauigkeit. Dissertation, Universität Kassel, Fachbereich Architektur, 1998.

[25] Grosso, M. CPCALC+-Calculation of Wind Pressure Coefficients on Buildings Versions 1.1. DOS and 1.1. WIN. Politecnico di Torino, Italy, 1995.

[26] Häckel, H. Meteorologie. Eugen Ulmer Stuttgart, 4. völlig überarb. Auflage, 1999.

[27] Haupt, W. Zur Simulation von auftriebsinduzierten Innenraumströmungen. Dissertation, Universität Kassel, Fachbereich Architektur, 2001.

[28] Hauser, G. und Maas, A. Auswirkungen von Fugen und Fehlstellen in Dampfsperren und Wärmedämmschichten. Deutsche Bauzeitschrift 24 (1992), H. 1, S. 97-100.

[29] Heiselberg, P., Svidt, K. und Nielsen, P. Characteristics of airflow from open windows. Building and Environment (2001), H. 36, S. 859-869.

[30] Hensen, J. On the thermal interaction of building structure and heating and ventilating system. Dissertation, Technische Universität Eindhoven, NL, 1991.

[31] Howell, S. und Potts, I. On the natural displacement ventilation flow through a full scale enclosure, by driven a source of bouyancy at floor level. Building Simulation, Rio de Janeiro, Brasilien, 7th int. IBPSA Conference, August 2001. 
[32] Hunt, G. und Linden, P. The fluid mechanics of natural ventilation - displacement ventilation by buoyancy-driven flows assistes by wind. Building and Environment (1999), H. 34, S. 707-720.

[33] Jischa, M. Konvektiver Impuls-, Wärme- und Stoffaustausch. Vieweg Verlag, Braunschweig, 1982.

[34] Knissel, J. Fenstergeometrie und Luftwechsel - verbessertes Modell. Arbeitskreis kostengünstige Passivhäuser, Protokollband Nr. 22, Passivhaus Institut, Darmstadt, 2003.

[35] Laporthe, S., Virgone, J. und Castanet, S. A comparative study of two tracer gases: $\mathrm{SF}_{6}$ and $\mathrm{N}_{2} \mathrm{O}$. Energy and Buildings (2001), H. 36, S. 313-312.

[36] Liddament, M. W. Air infiltration calculation techniques - an applications guide. IEA Air Infiltration And Ventilation Center (AIVC), Bracknell, UK, 1986.

[37] Maas, A. Experimentelle Quantifizierung des Luftwechsels bei Fensterlüftung. Dissertation, Universität Kassel, Fachbereich Architektur, 1995.

[38] Maas, A., Schmidt, D. und Hauser, G. Experimentelle Untersuchungen zum Luftaustausch bei Querlüftung. wksb (1998), H. 41, S. 39-45.

[39] Meyer, C. Bewertung thermischer Behaglichkeit mittels Strömungssimulation. Dissertation, Universität Kassel, Fachbereich Technische Gebäudeausrüstung, 1999.

[40] Nolte, C. Zum Lüftungsverhalten von Doppelfassaden. Fortschritt-Berichte VDI, Nr. 158, VDI Verlag GmbH, Düsseldorf, 1999.

[41] Oertel, H. Prandtl - Führer durch die Strömungslehre. Vieweg, Braunschweig, 10. Auflage, 1982.

[42] Oppermann, J. Untersuchung der Sensitivität von Heizung-/Lüftungsanlagen in Niedrigenergiehäusern. Dissertation, Universität Kassel, Fachbereich Technische Gebäudeausrüstung, 2003.

[43] Peppes, A., Santamouris, M. und Asimakopoulos, D. Experimental and numerical study of bouyancy-driven stairwell flow in a three storey building. Energy and Buildings (2002), H. 37, S. 497-506.

[44] Prandtl, L., Oswatitsch, K. und Wieghardt, K. Führer durch die Strömungslehre. Vieweg, Braunschweig, 8. Auflage, 1984.

[45] Press, W. Numerial recipes in C: the art of scientific computing. Press Syndicate of the University of Cambridge, 2. Auflage, 1992.

[46] Raatschen, W. Tracergasmessungen in der Gebäudetechnik - Teil 1. Gesundheits-Ingenieur (1995), H. 2, S. 78-87. 
[47] Reid, R., Prausnitz, J. M. und Poling, B. The Properities of Gases and Liquids. McGraw-Hill Book Co., Singapore, 4. Auflage, 1988.

[48] Richter, W. Handbuch der thermischen Behaglichkeit - Heizperiode. Schriftenreihe der Bundesanstalt für Arbeitsschutz und Arbeitsmedizin, 2003.

[49] Riederer, R., Coulturier, S. und Marchio, D. Influence of sensor position in building thermal control: criteria for zone models. Clima 2000, Neapel, September 2001.

[50] Rösler, M. Aufbau eines Meßsystems zur Bestimmung der Gasdiffusion durch Zwangsentlüftungselementen. Projektarbeit, Universität Kassel, Fachgebiet Bauphysik, 1997.

[51] Rösler, M. Aufbau eines Meßsystems zur Ermittlung des Luftwechsels bei natürlicher Fensterlüftung (Querlüftung). Diplomarbeit, Universität Kassel, Fachgebiet Bauphysik, 1998.

[52] Schnieders, J. Ein vereinfachtes Verfahren zur Abschätzung des sommerlichen Luftwechsels. Arbeitskreis kostengünstige Passivhäuser, Protokollband Nr. 22, Passivhaus Institut, Darmstadt, 2003.

[53] Sedlbauer, K., Gabrio, T. und Krus, M. Schimmelpilze - Gesundheitsgefährdung und Vorhersage. Gesundheits-Ingenieur 123 (2002), H. 6, S. 285336.

[54] Synergy Software (PSC Inc.), 2457 Perkiomen Avenue, Reading, PA 19606. Kaleidagraph Reference Guide, 1988-1996.

[55] Taylor, J. An Introduction to Error Analysis - The Study of Uncertainties in Physical Measurements, 2. Auflage. University Science Books, Sausalito, California, 1997.

[56] van der Maas, J. et al. Air flow through large openings in buildings. Annex 20: Air flow patterns within buildings, Subtask 2: Airflow between Zones, 1992.

[57] Weber, A. Modell für natürliche Lüftung durch Kippfenster. TRNSYS Usertag in Stuttgart, EMPA, CH-8600 Dübendorf, 1997.

[58] Wong, N. und Chin, H. An evaluation exercise of a wind pressure distribution model. Energy and Buildings (2002), H. 34, S. 291-309.

[59] Zeidler, O. Grenzen der thermischen Last bei Fensterlüftung in Bürogebäuden. Fortschritt-Berichte VDI, Reihe 19, Nr. 134, VDI Verlag GmbH, Düsseldorf, 2001.

[60] Zierep, J. Ähnlichkeitsgesetze und Modellregeln der Strömungslehre. C. Braun, Karlsruhe, 2. überarb. Auflage, 1982.

[61] Zierep, J. Grundzüge der Strömungslehre. C. Braun, Karlsruhe, 2. überarb. Auflage, 1982. 
[62] Ziller, C. Modellversuch und Berechnungen zur Optimierung der natürlichen Lüftung durch Doppelfassaden. Schriftenreihe Stahlbau - RWTH Aachen, Heft 44, Schaker Verlag, Aachen, 1999.

[63] Zürcher, C. und Frank, T. Bauphysik-Bau und Energie, Bd. 2. B.G. Teubner, Stuttgart, 1998. 


\section{Anhang}

\subsection{Prozentuale Abweichung der einzelnen Varianten}

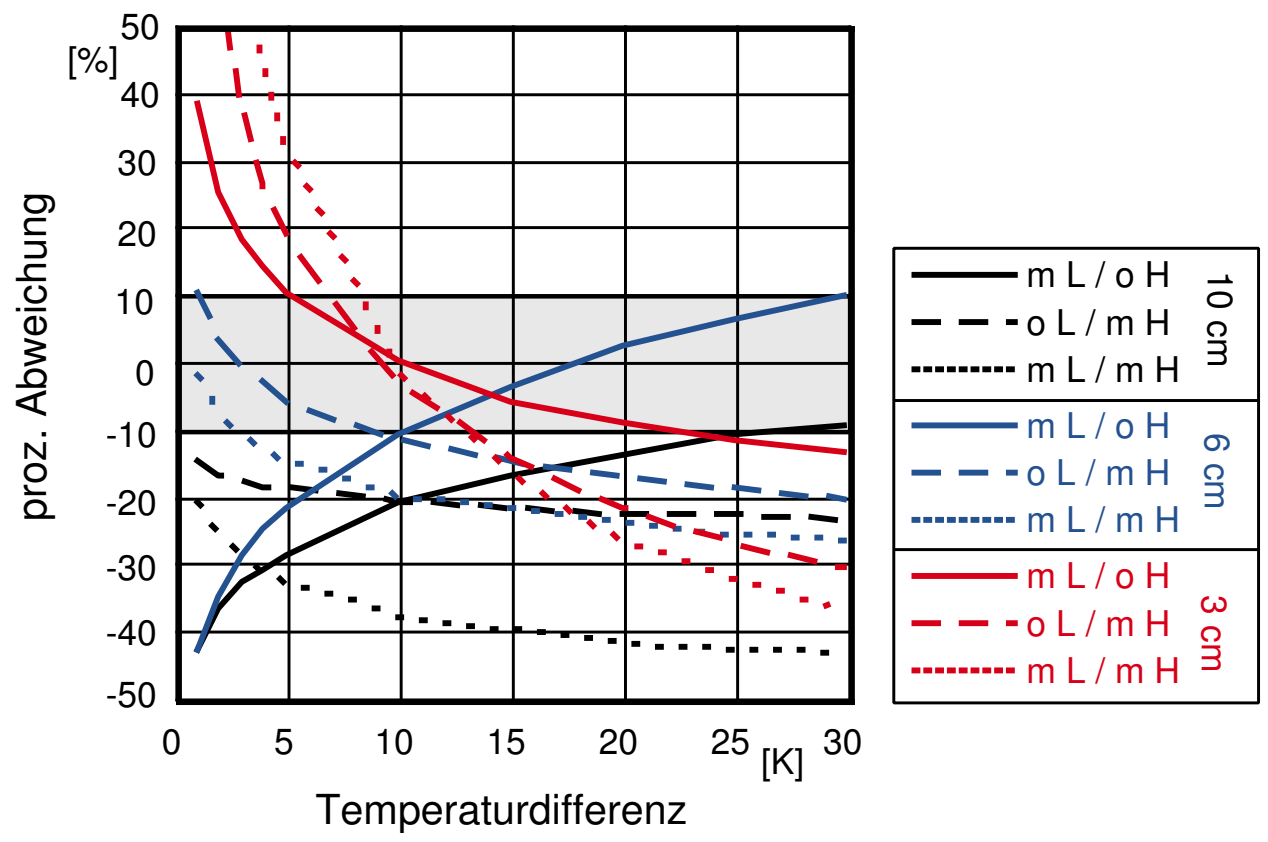

Bild 38: Prozentuale Abweichung der einzelnen Varianten von Variante 'ohne Laibung / ohne Heizung' in Abhängigkeit von der Temperaturdifferenz (Kippweite $10 \mathrm{~cm}$ ).

\subsection{Temperaturverläufe bei der Kippweite von $10 \mathrm{~cm}$}

Variante 'ohne Laibung / ohne Heizung': Bild 39

Variante 'mit Laibung / ohne Heizung': Bild 40

Variante 'mit Laibung / mit Heizung': Bild 41

alle Varianten: Boden und Deckenoberflächentemperaturen: Bild 42

\subsection{Temperaturverläufe bei der Kippweite von $6 \mathrm{~cm}$}

Variante 'mit Laibung / ohne Heizung': Bilder 43 und 44

Variante 'mit Laibung / mit Heizung': Bilder 45 und 46

beide Varianten: Positionen A/B: Bild 47

beide Varianten: Boden und Deckenoberflächentemperaturen: Bild 48 


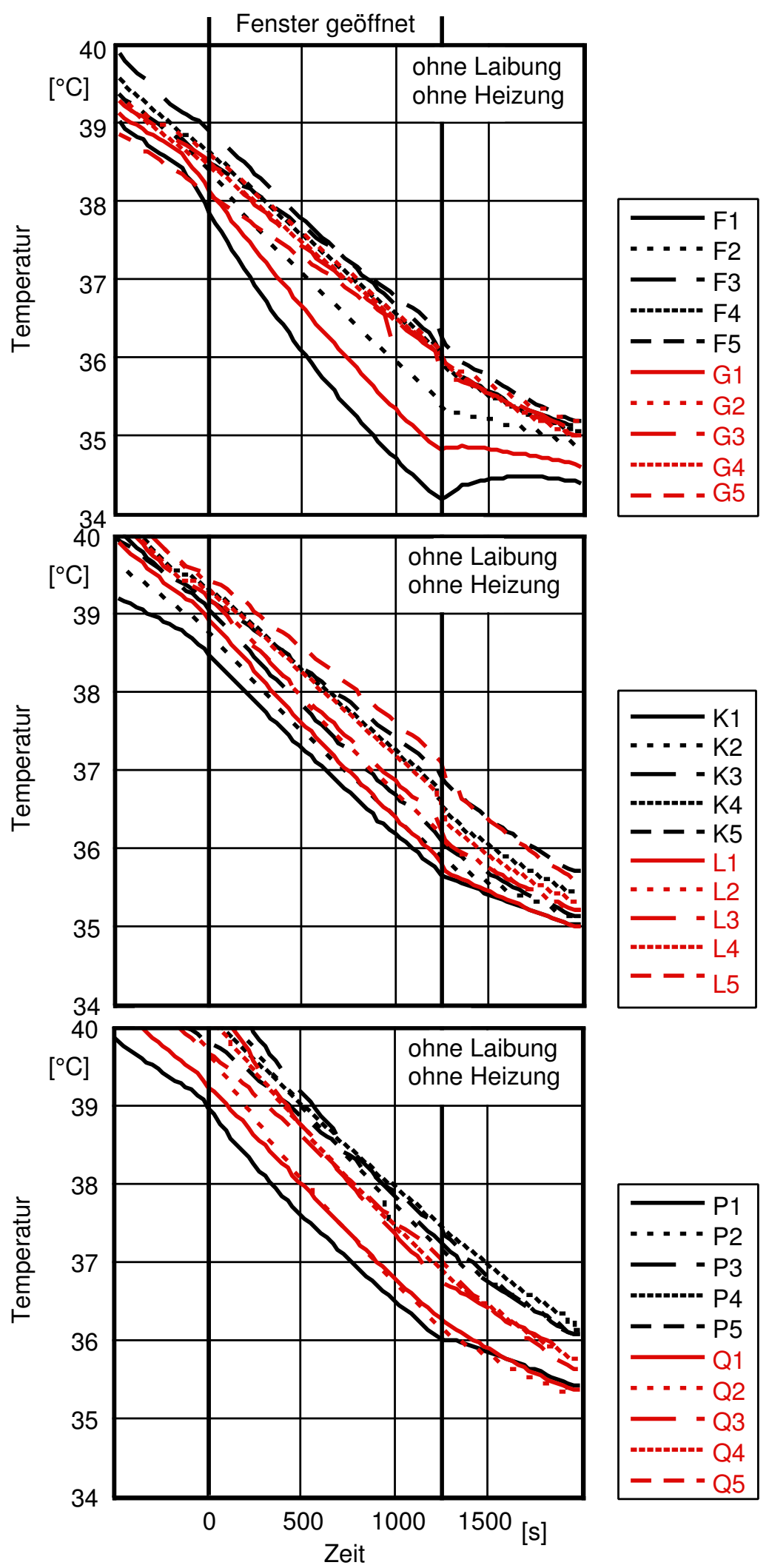

Bild 39: Zeitlicher Verlauf der Wandtemperaturen für die Variante 'ohne Laibung / ohne Heizung' an den Positionen F/G, K/l und $\mathrm{P} / \mathrm{Q}$ (Kippweite $10 \mathrm{~cm}$ ). 

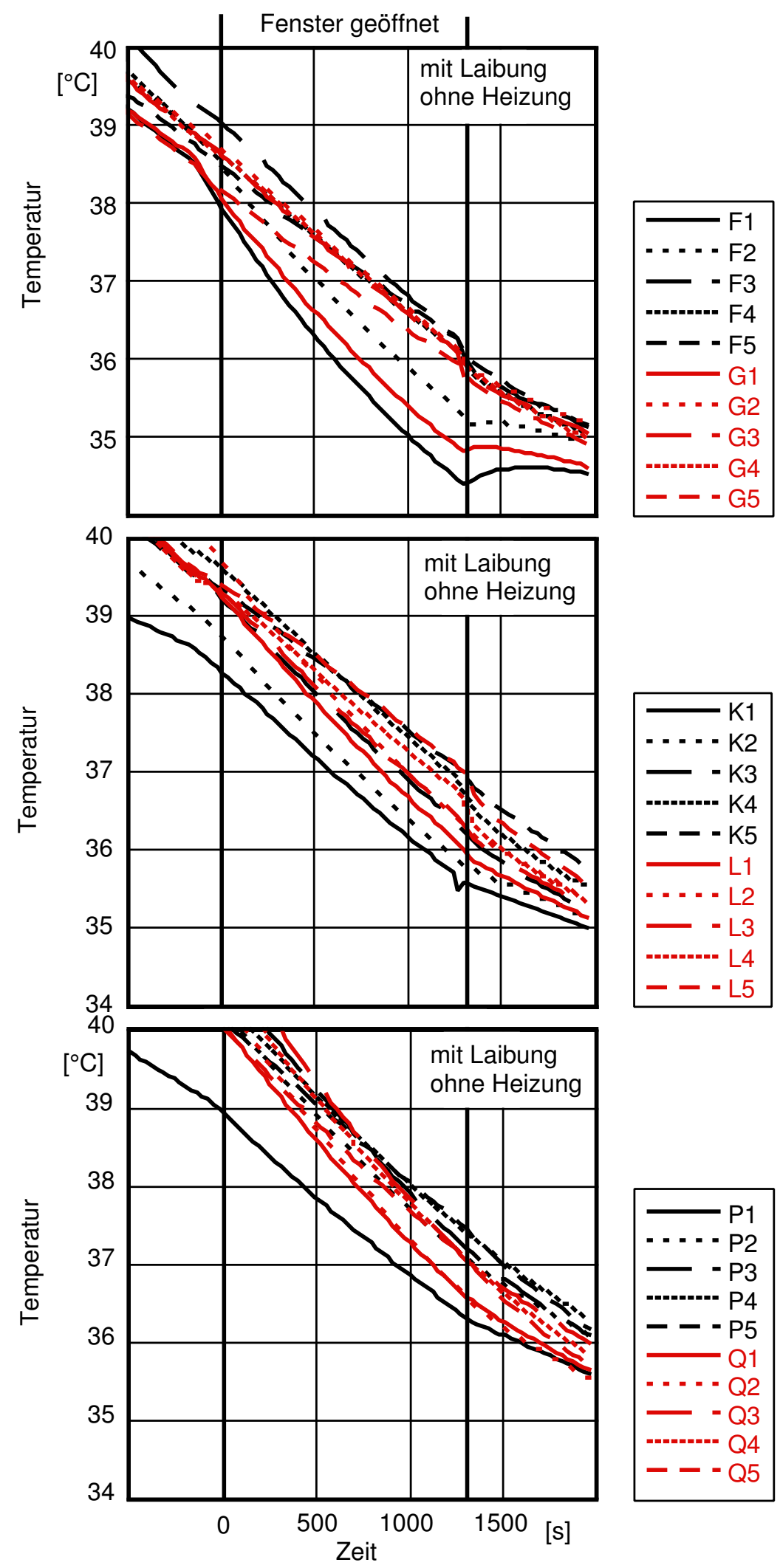

Bild 40: Zeitlicher Verlauf der Wandtemperaturen für die Variante 'mit Laibung / ohne Heizung' an den Positionen F/G, K/l und P/Q (Kippweite $10 \mathrm{~cm}$ ). 


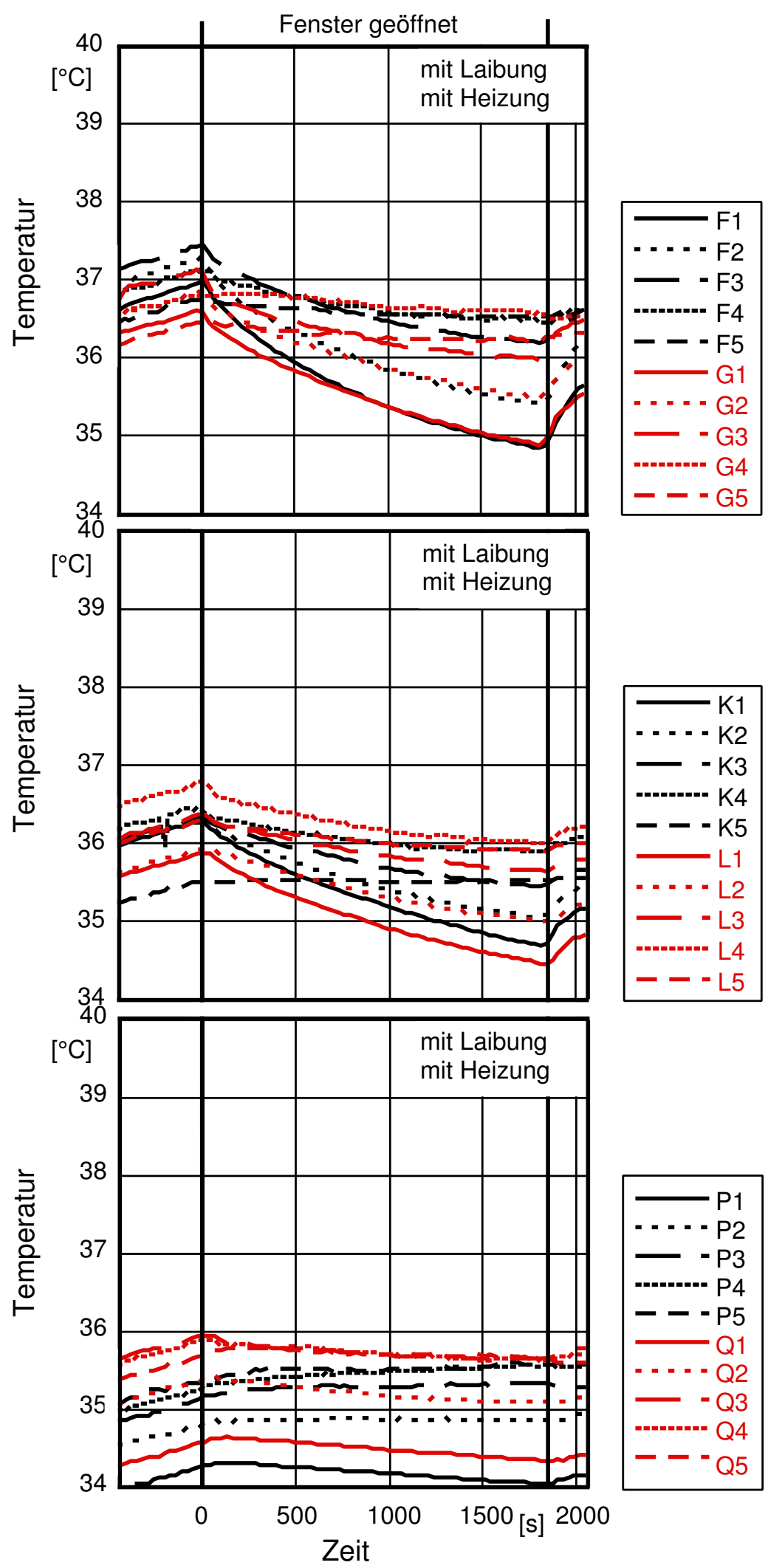

Bild 41: Zeitlicher Verlauf der Wandtemperaturen für die Variante 'mit Laibung / mit Heizung' an den Positionen F/G, K/l und P/Q (Kippweite $10 \mathrm{~cm}$ ). 

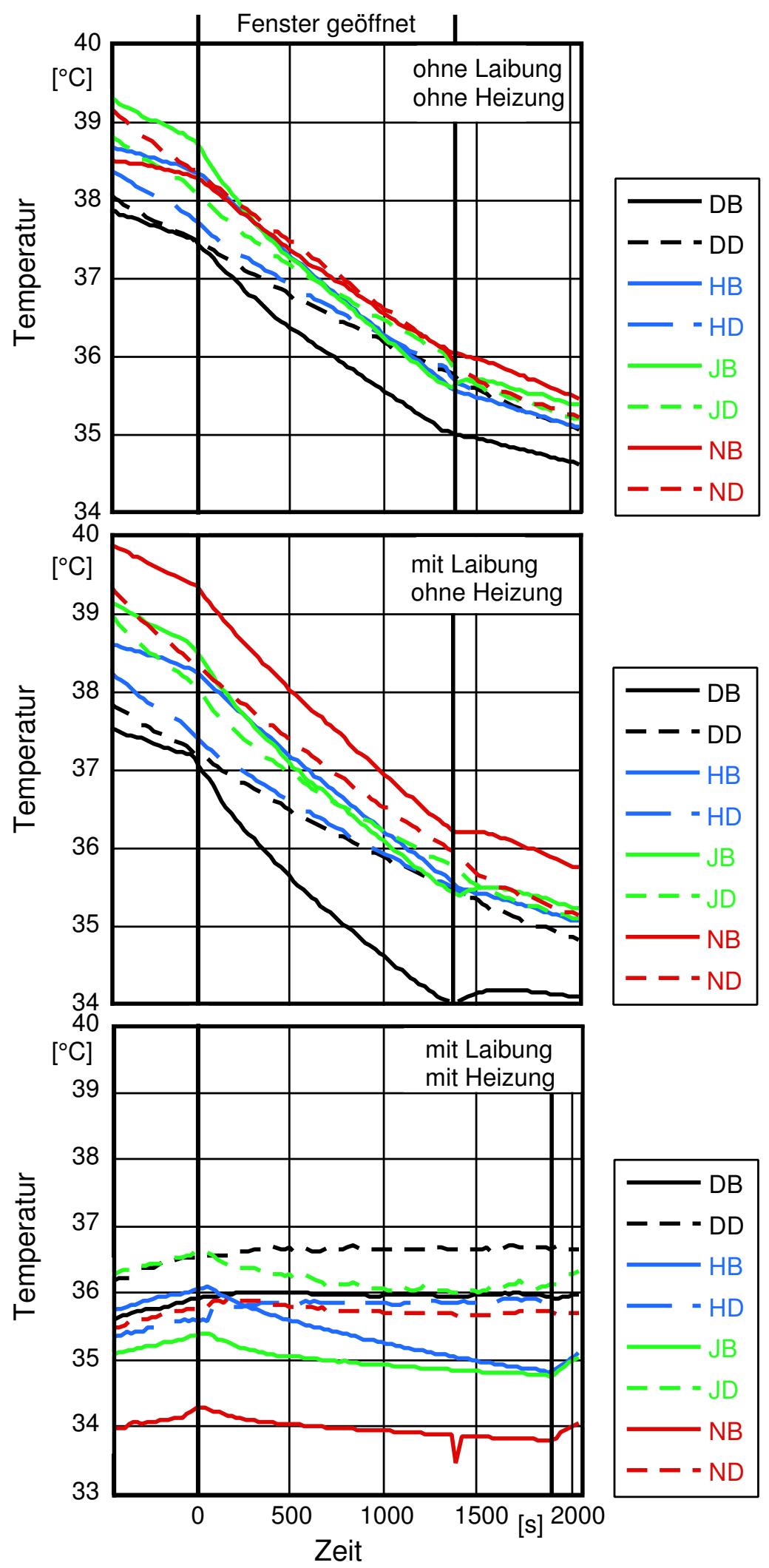

Bild 42: Zeitlicher Verlauf der Oberflächentemperaturen am Boden und der Decke für alle Varianten (Kippweite $10 \mathrm{~cm}$ ). 


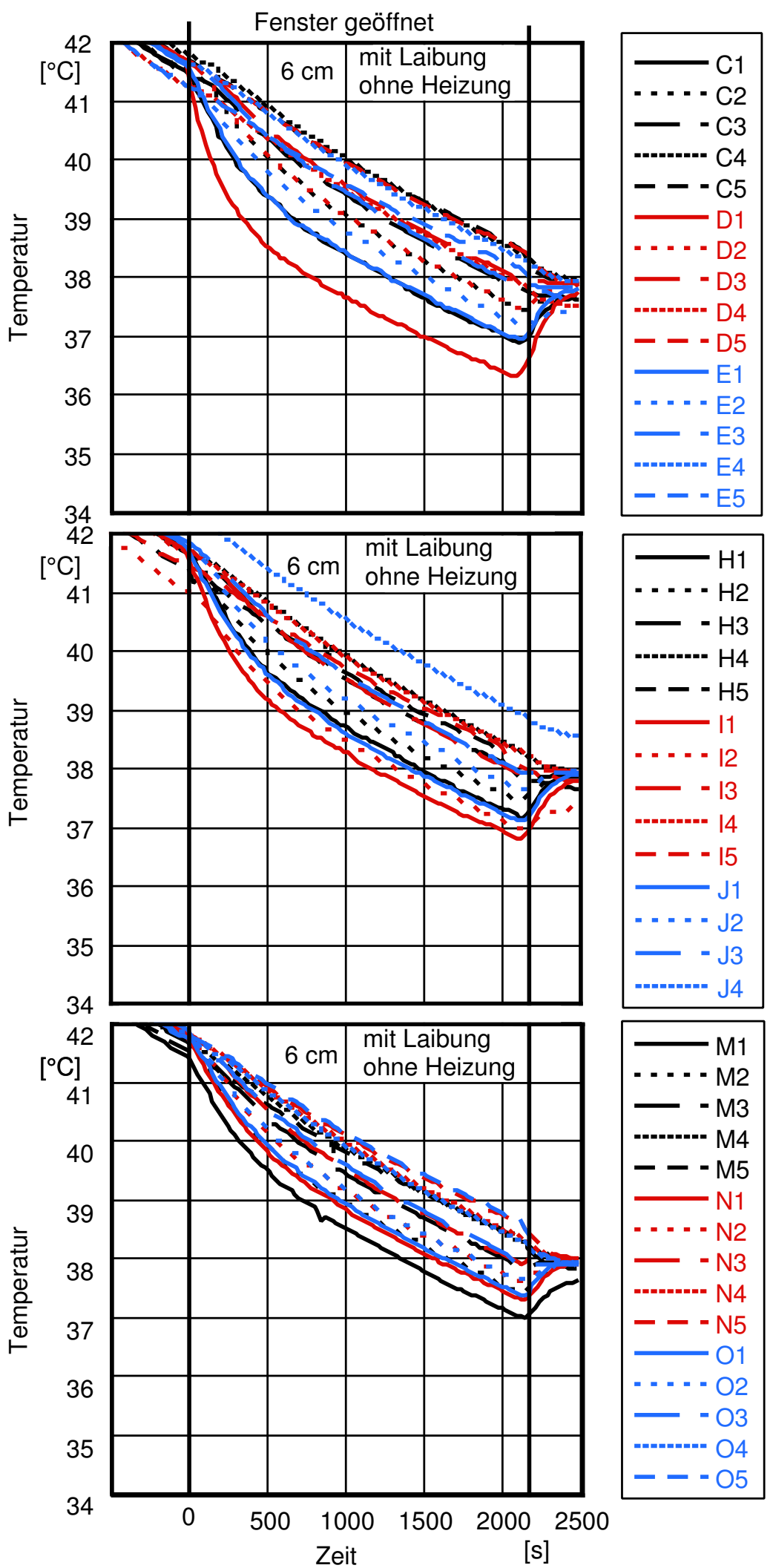

Bild 43: Zeitlicher Verlauf der Lufttemperaturen an den Messstellen C/D/E, H/I/J und M/N/O für die Variante 'mit Laibung / ohne Heizung' (Kippweite $6 \mathrm{~cm}$ ). 


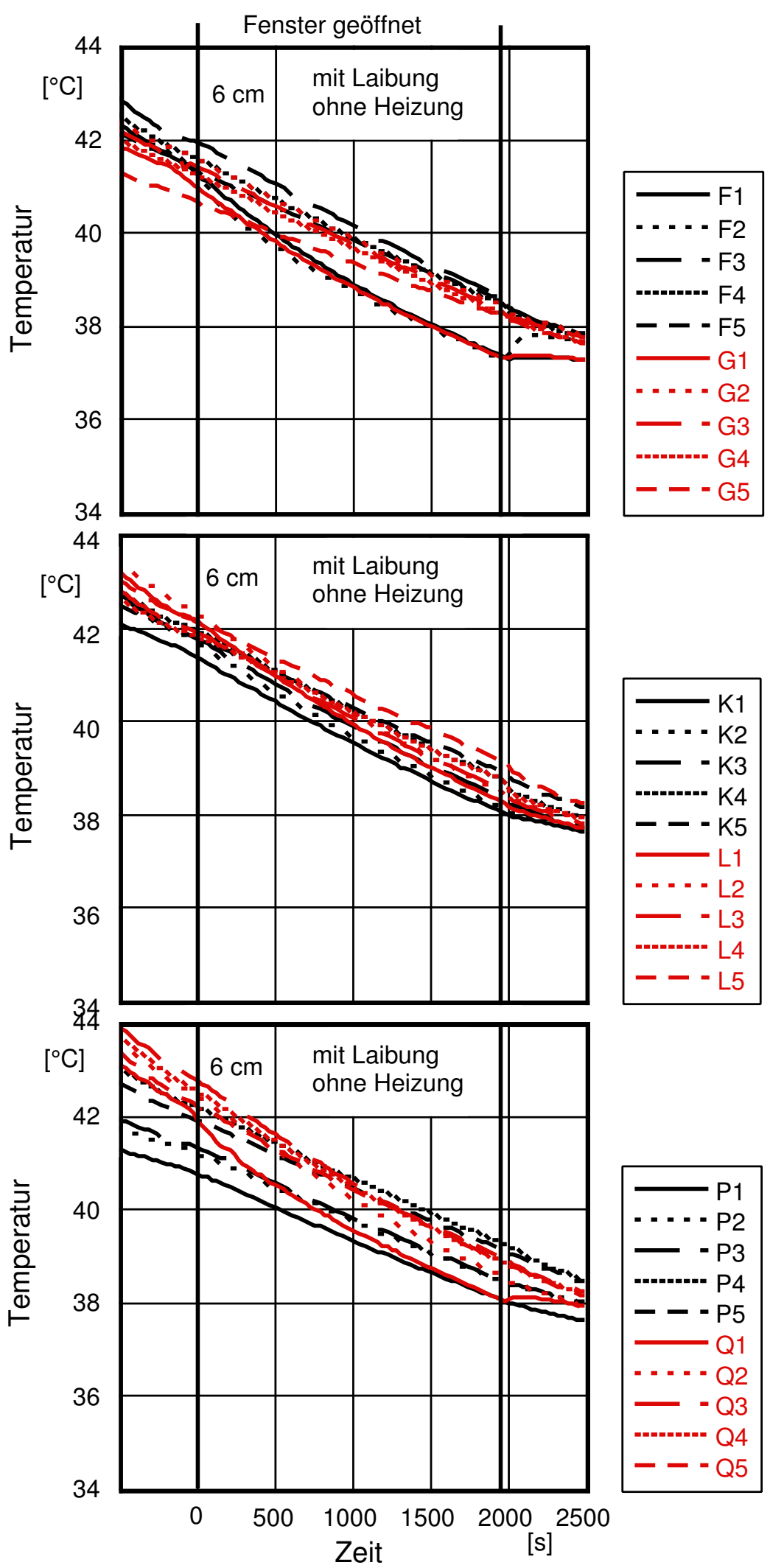

Bild 44: Zeitlicher Verlauf der Lufttemperaturen an den Messstellen F/G, K/L und P/Q für die Variante 'mit Laibung / ohne Heizung' (Kippweite $6 \mathrm{~cm}$ ). 


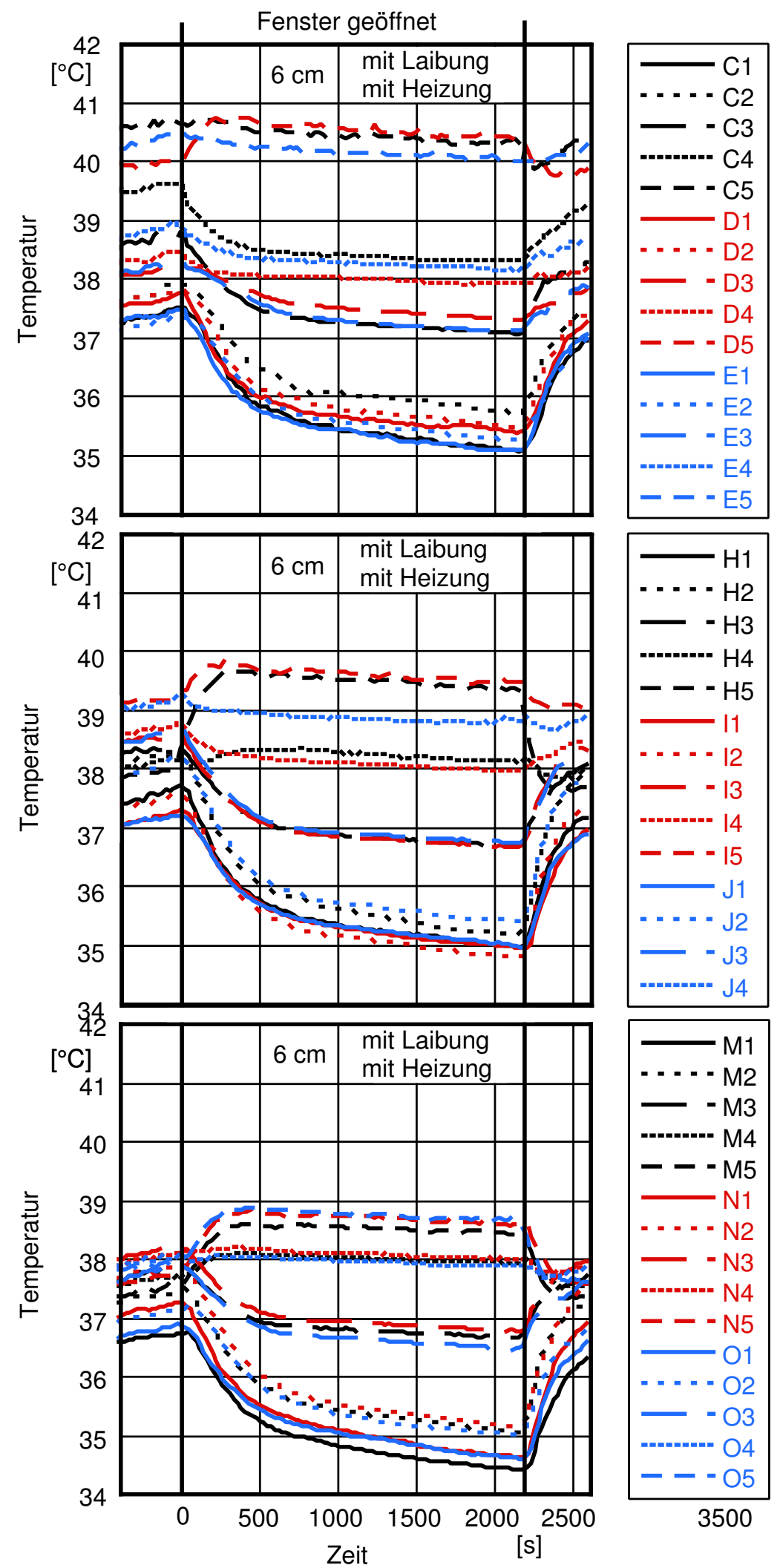

Bild 45: Zeitlicher Verlauf der Lufttemperaturen an den Messstellen C/D/E, H/I/J und M/N/O für die Variante 'mit Laibung / mit Heizung' (Kippweite $6 \mathrm{~cm}$ ). 


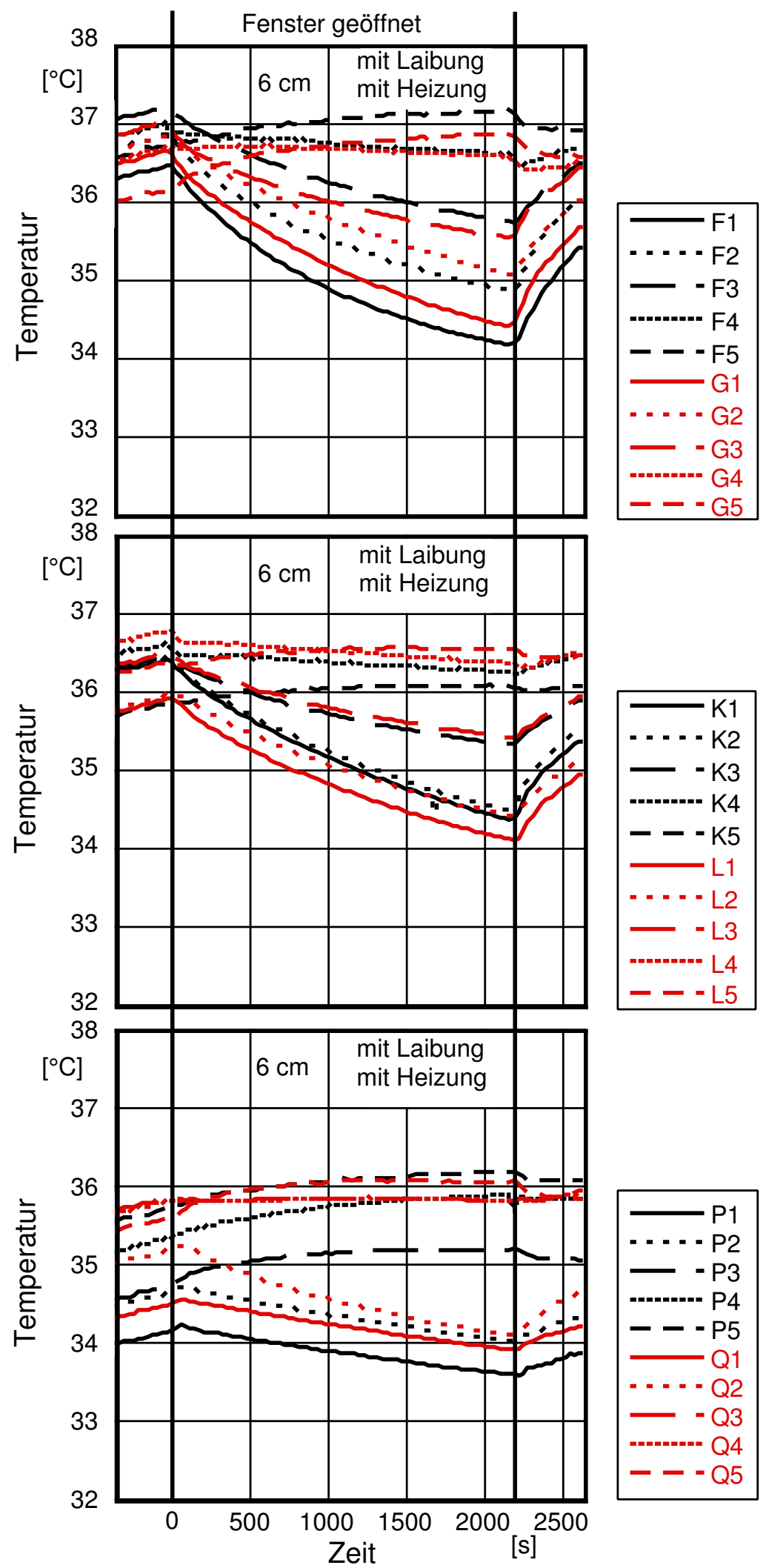

Bild 46: Zeitlicher Verlauf der Lufttemperaturen an den Messstellen F/G, K/L und P/Q für die Variante 'mit Laibung / mit Heizung' (Kippweite $6 \mathrm{~cm}$ ). 


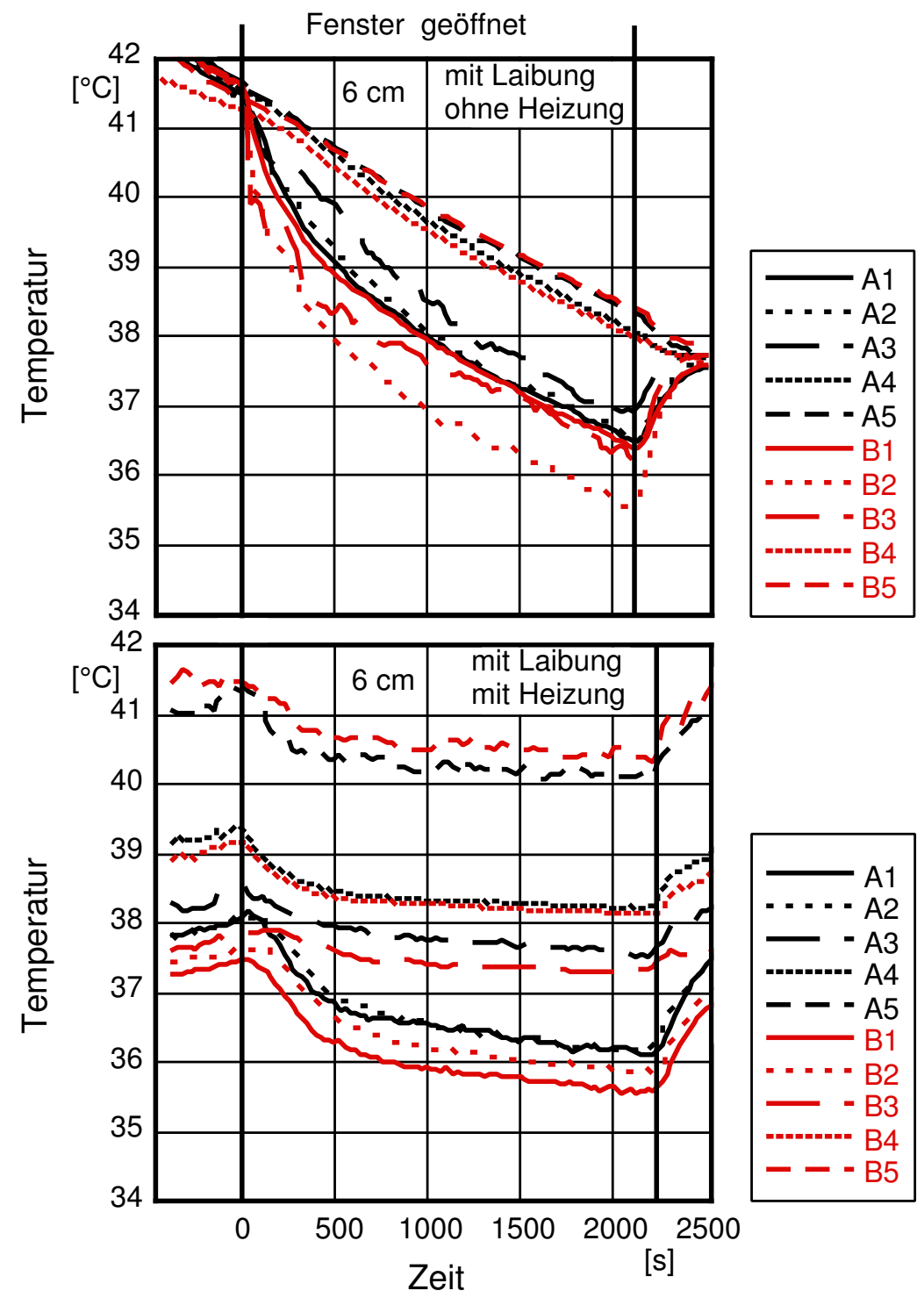

Bild 47: Zeitlicher Verlauf der Lufttemperaturen an den Messstellen A/B für die Variante 'mit Laibung / ohne Heizung' bzw. 'mit Laibung / mit Heizung' (Kippweite $6 \mathrm{~cm}$ ). 


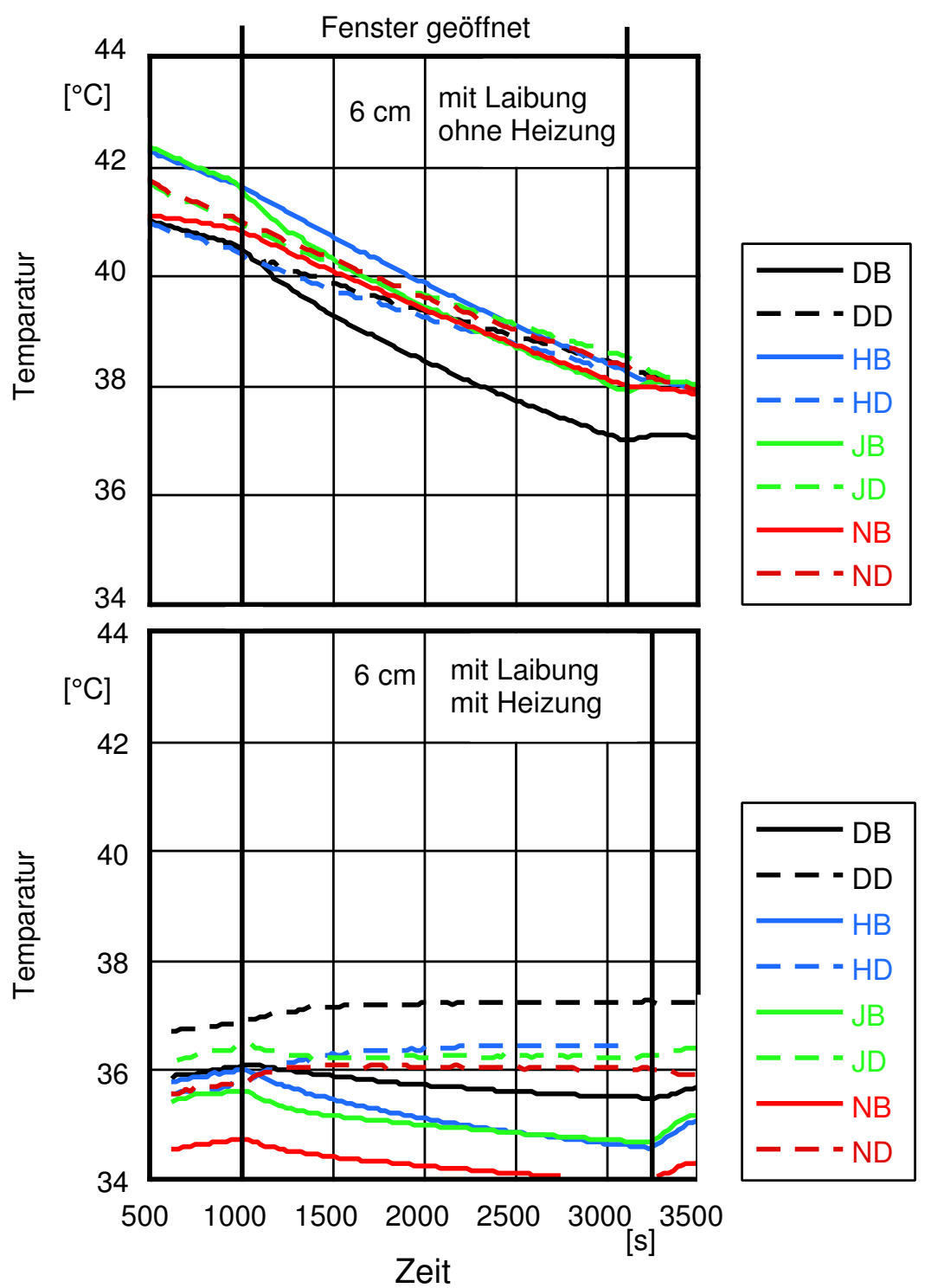

Bild 48: Zeitlicher Verlauf der Oberflächentemperaturen an Bonden und Decke für die Variante 'mit Laibung / ohne Heizung' bzw. 'mit Laibung / mit Heizung' (Kippweite $6 \mathrm{~cm}$ ). 


\subsection{Temperaturverläufe bei der Kippweite von $3 \mathrm{~cm}$}

Variante 'ohne Laibung / ohne Heizung': Bilder 49 und 50

Variante 'mit Laibung / ohne Heizung': Bilder 51 und 52

Variante 'mit Laibung / mit Heizung': Bilder 53 und 54

alle Varianten: Positionen A/B: Bild 55

alle Varianten: Boden und Deckenoberflächentemperaturen: Bild 56 


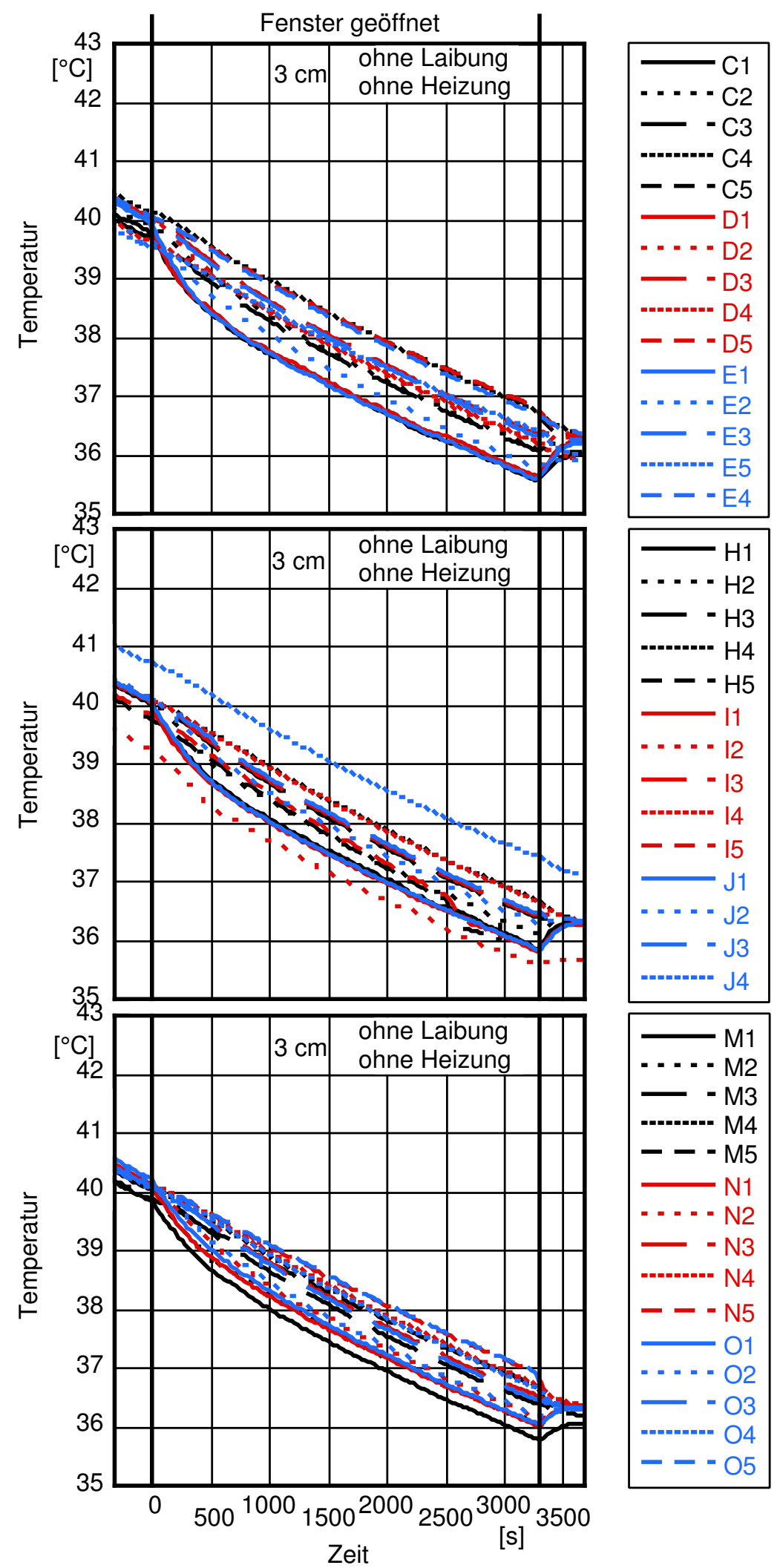

Bild 49: Zeitlicher Verlauf der Lufttemperaturen an den Messstellen C/D/E, H/I/J und M/N/O für die Variante 'ohne Laibung / ohne Heizung' (Kippweite $3 \mathrm{~cm}$ ). 

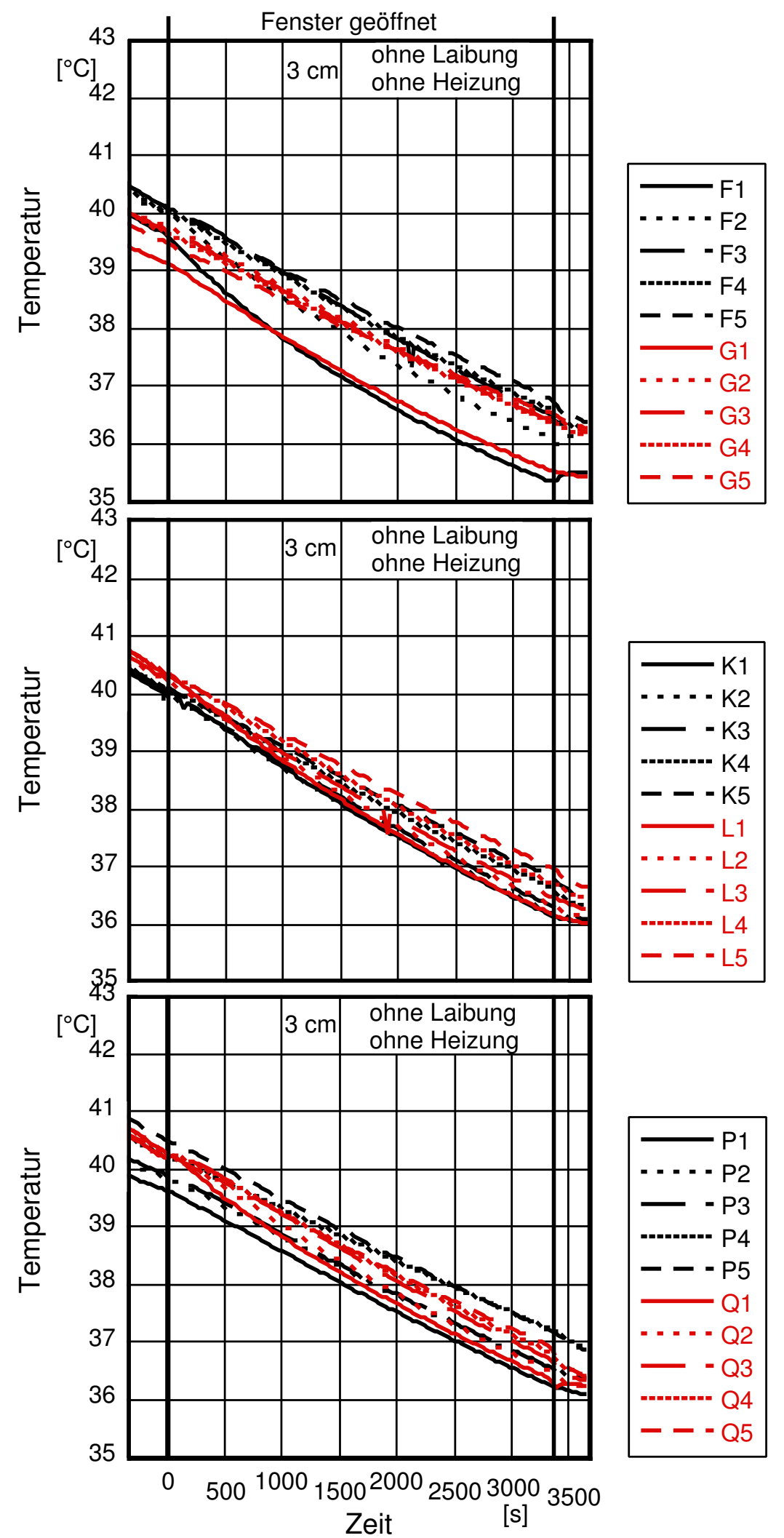

Bild 50: Zeitlicher Verlauf der Lufttemperaturen an den Messstellen F/G, K/L und P/Q für die Variante 'ohne Laibung / ohne Heizung' (Kippweite $3 \mathrm{~cm}$ ). 

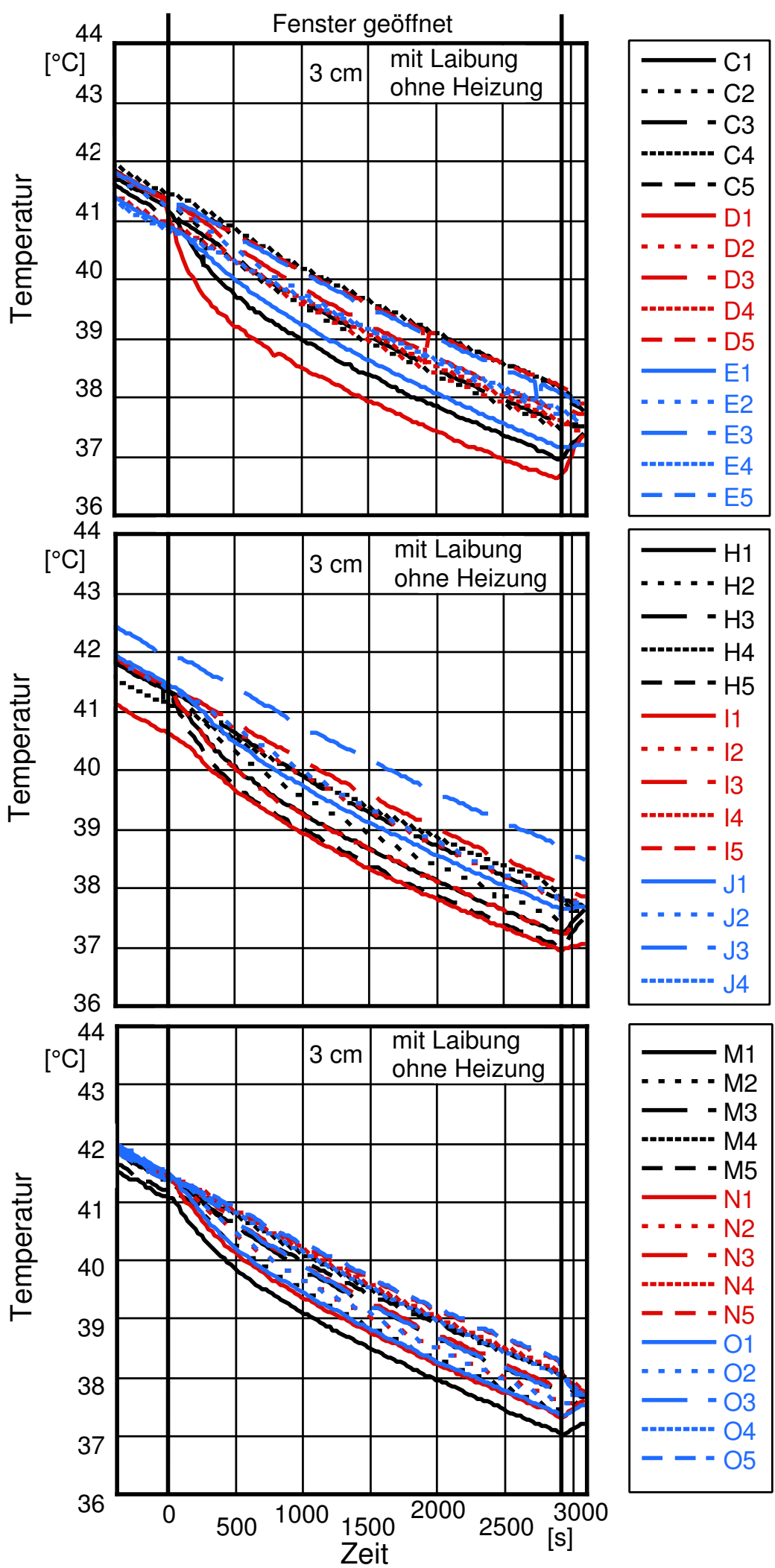

Bild 51: Zeitlicher Verlauf der Lufttemperaturen an den Messstellen C/D/E, H/I/J und M/N/O für die Variante 'mit Laibung / ohne Heizung' (Kippweite $3 \mathrm{~cm}$ ). 


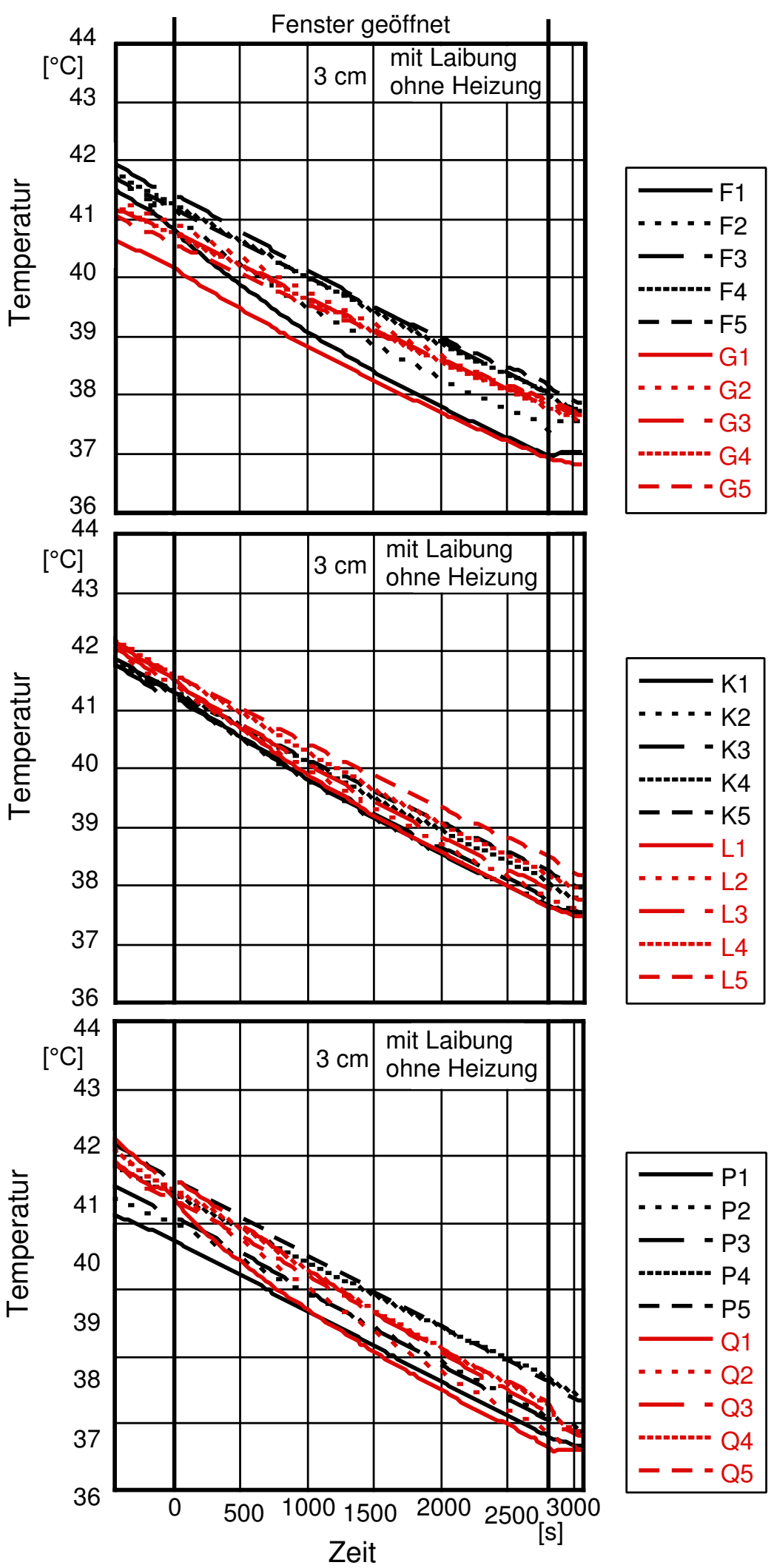

Bild 52: Zeitlicher Verlauf der Lufttemperaturen an den Messstellen F/G, K/L und P/Q für die Variante 'mit Laibung / ohne Heizung' (Kippweite $3 \mathrm{~cm}$ ). 


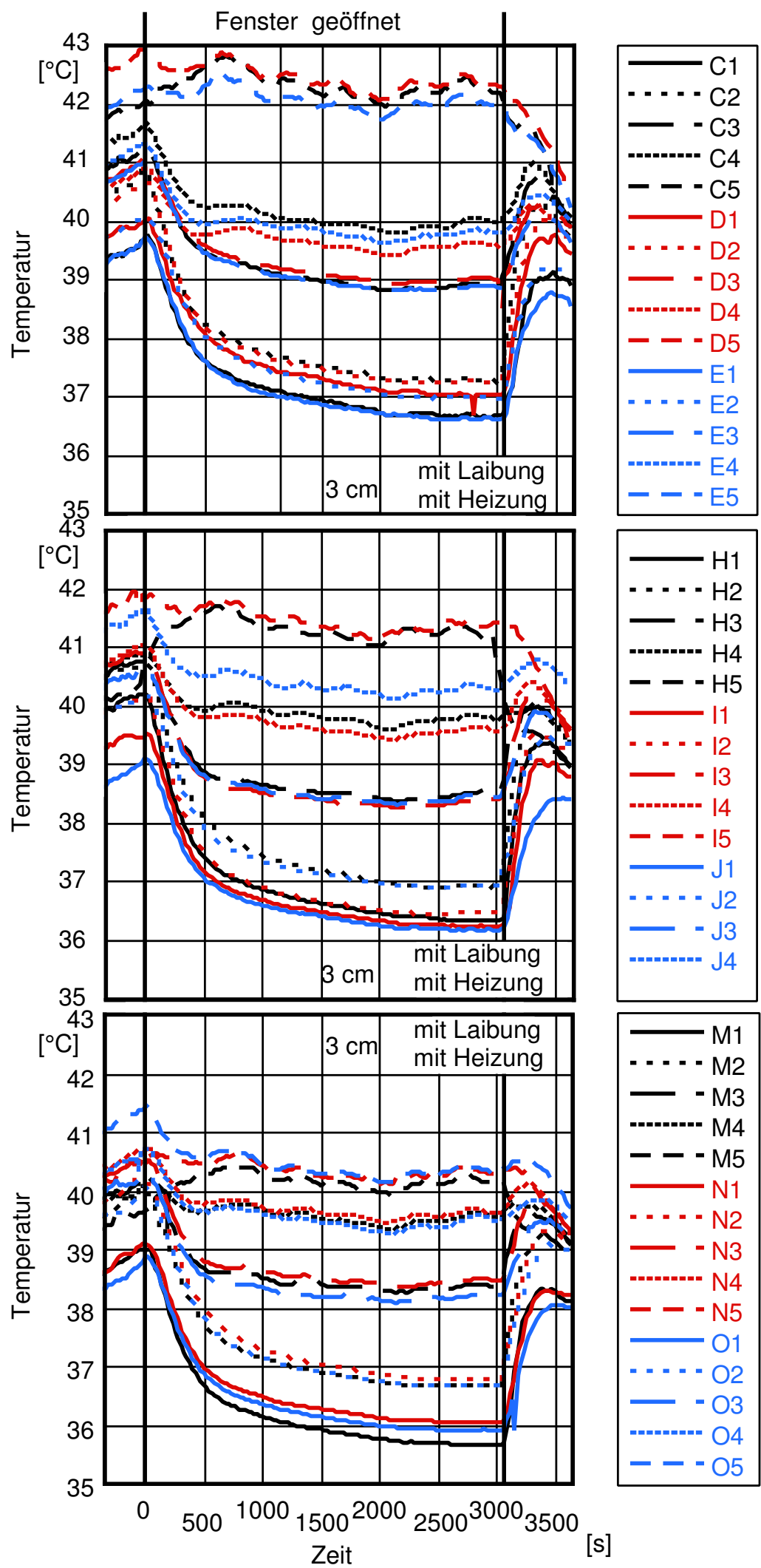

Bild 53: Zeitlicher Verlauf der Lufttemperaturen an den Messstellen $\mathrm{C} / \mathrm{D} / \mathrm{E}, \mathrm{H} / \mathrm{I} / \mathrm{J}$ und M/N/O für die Variante 'mit Laibung / mit Heizung' (Kippweite $3 \mathrm{~cm}$ ). 

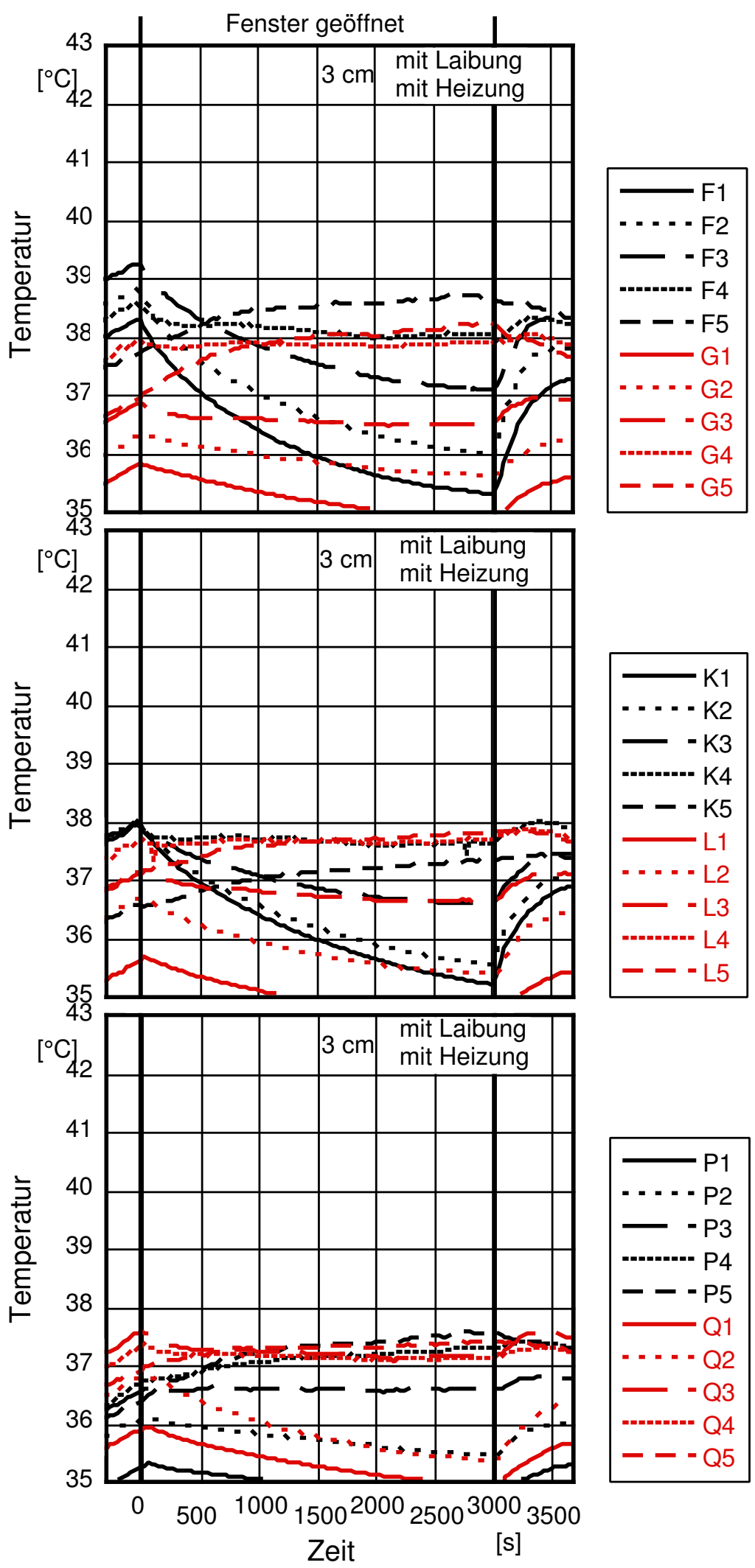

Bild 54: Zeitlicher Verlauf der Lufttemperaturen an den Messstellen F/G, K/L und P/Q für die Variante 'mit Laibung / mit Heizung' (Kippweite $3 \mathrm{~cm}$ ). 


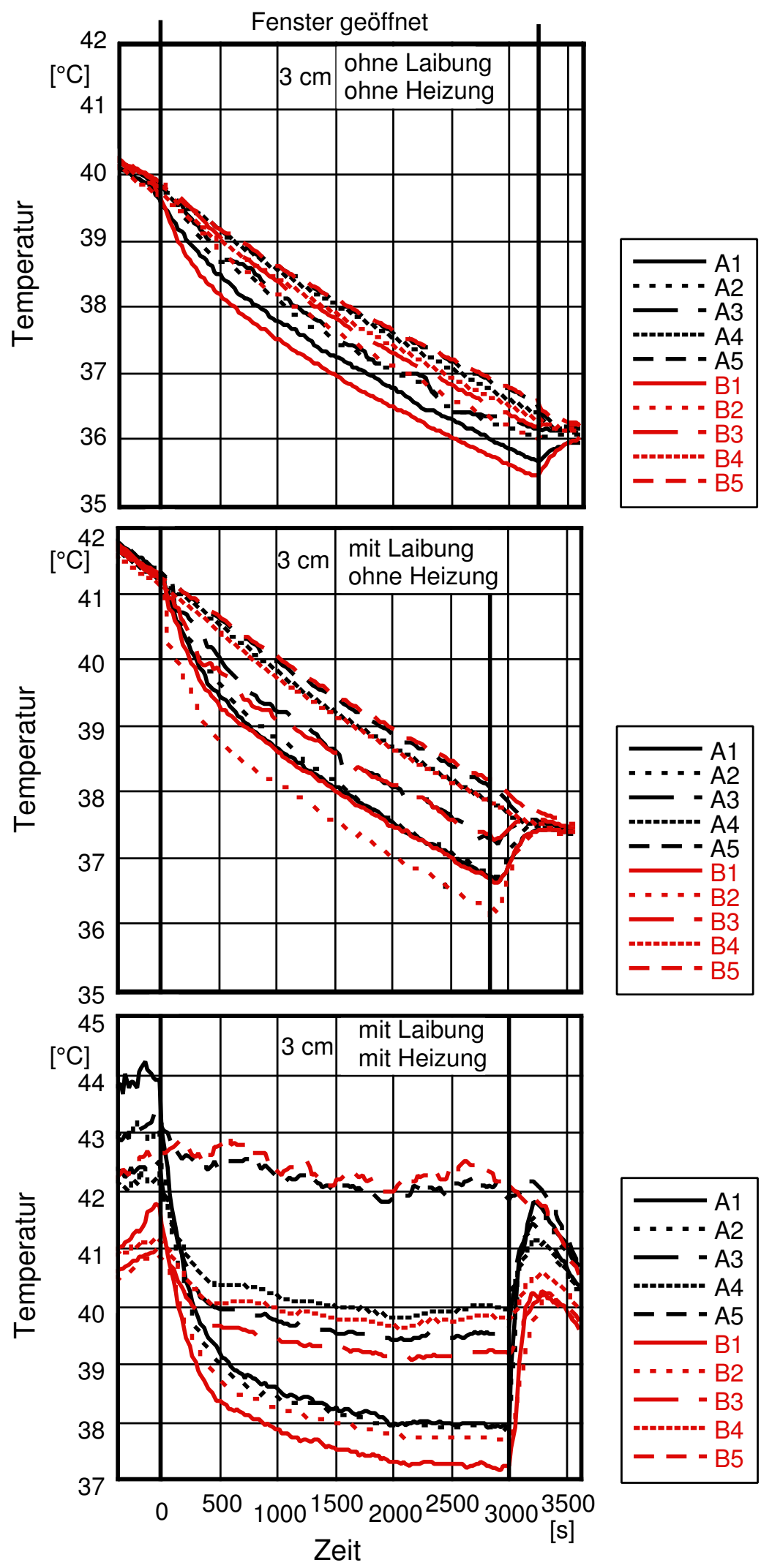

Bild 55: Zeitlicher Verlauf der Lufttemperaturen an den Messstellen A/B für alle Varianten (Kippweite $3 \mathrm{~cm}$ ). 

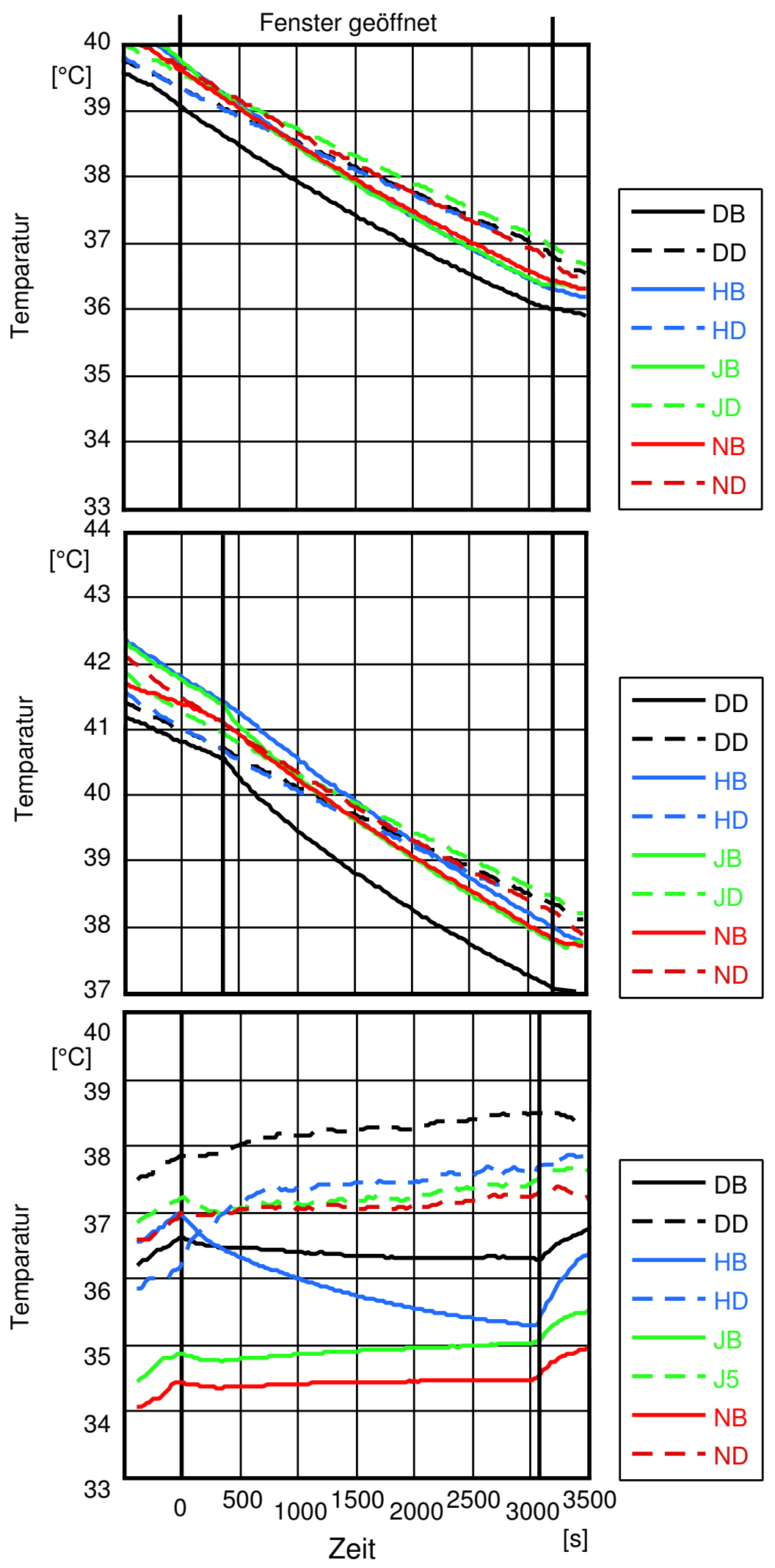

Bild 56: Zeitlicher Verlauf der Oberflächentemperaturen an Boden und Decke für alle Varianten (Kippweite $3 \mathrm{~cm}$ ). 


\subsection{Korrelationskoeffizient}

Die Messwerte werden mit dem Programm Kaleidagraph durch einen Powerfit nach Gl. 42 angepasst.

$$
y=a x^{b}
$$

Den angegebenen Korrelationskoeffizienten liegt gemäß [54, 45] die folgende Bestimmungsgleichung zugrunde

$$
r=\frac{\sum_{i}^{N}\left(x_{i}-\bar{x}\right)\left(y_{i}-\bar{y}\right)}{\sqrt{\sum_{i}^{N}\left(x_{i}-\bar{x}\right)^{2}} \sqrt{\sum_{i}^{N}\left(y_{i}-\bar{y}\right)^{2}}}
$$

mit

r Korrelationskoeffizient (least squares curves fits)

$\mathrm{N}$ Anzahl der Wertepaare

$x_{i} \quad$ Werte für $\Delta \mathrm{T}$

$y_{i} \quad$ Werte für $\dot{V}$

$\bar{x} \quad$ Mittelwert aller $x_{i}$

$\bar{y} \quad$ Mittelwert aller $y_{i}$ 


\section{Bild- und Tabellenverzeichnis}

\section{Abbildungsverzeichnis}

1 Wind- und Temperatureinfluss . . . . . . . . . . . . 5

2 Ein- und Ausströmbereich eines Kippfensters. . . . . . . . . . . . . 11

3 Visualisierung der ein- und ausströmenden Luft. . . . . . . . . . . . 12

$4 \quad$ Strömung durch ein Kippfenster. . . . . . . . . . . . . . . . . . . 12

$5 \quad$ Kippfenster mit Laibung . . . . . . . . . . . . . . . . . 13

6 Vergleich verschiedener Ansätze . . . . . . . . . . . . . . 18

7 Skizze des Modellraums im Labor . . . . . . . . . . . . . . . . 21

8 Versuchsanordnung im Labor und Modellraum . . . . . . . . . 22

9 Anordnung der Temperaturfühler im Modellraum . . . . . . . . . 23

10 Zuluftvolumenstrom bei $10 \mathrm{~cm}$ Kippweite . . . . . . . . . . . . . . 29

11 Zuluftvol. ber der Wurzel der Temperaturdifferenz $(10 \mathrm{~cm})$. . . . 30

12 Vergleich unterschiedlicher Laibungstiefen . . . . . . . . . . . . . 30

13 Zuluftvolumenstrom bei $6 \mathrm{~cm}$ Kippweite . . . . . . . . . . . . 33

14 Zuluftvolumenstrom bei $3 \mathrm{~cm}$ Kippweite . . . . . . . . . . . . . . 34

15 Anordnung der Heizung im Raum. . . . . . . . . . . . . . . . . 36

16 Anordnung mit Ventilatoren. . . . . . . . . . . . . . 37

17 Zuluftvolumenstrom unter Berücksichtigung einer Gardine. . . . . . 38

18 Vergleich der Messwerte mit Ansätzen aus der Literatur. . . . . . . 40

19 Lufttemp.: Variante 'ohne Laibung / ohne Heizung' $(10 \mathrm{~cm}) . \ldots 42$

20 Lufttemp.: Variante 'mit Laibung / ohne Heizung' $(10 \mathrm{~cm})$. . . . . . 44

21 Lufttemp.: Variante 'mit Laibung / mit Heizung' $(10 \mathrm{~cm})$. . . . . . . 46

22 Temperaturverlauf der Positionen A/B $(10 \mathrm{~cm}) . \ldots . \ldots . . . . .48$

23 Geometrie des CFD-Modells . . . . . . . . . . . . . . . 52

24 Seitenansicht des CFD-Modells mit Gitterpunkten . . . . . . . . . . 53

25 Gitterpunkte in der Öffnungsfläche des Kippfensters . . . . . . . . . 53

26 Vergleich der Zuluftvolumenströme . . . . . . . . . . . 58

27 Volumenströme bestimmt aus dem Massenstrom/Tracergas. . . . . . 59

28 Lufttemperaturverteilung mit konst. Wandtemperatur. . . . . . . . . 61 
29 Lufttemperaturverteilung mit freier Wandtemperatur . . . . . . . . 63

30 Stromlinien nach $20 \mathrm{~s} . \ldots \ldots 5$

31 Stromlinien nach $40 \mathrm{~s} . \ldots \ldots 66$

32 Stromlinien nach 59 s. . . . . . . . . . . . . . . 67

33 Stromlinien nach 122 s. . . . . . . . . . . . . . . . 68

34 Verschiedene Öffnungswinkel . . . . . . . . . . . . 70

35 Verschiedene Bereiche der Dreiecke . . . . . . . . . . . . . 74

36 Cd-Werte in Abhängigkeit von der Kippweite . . . . . . . . . . . 77

37 Verhältnis der Volumenströme . . . . . . . . . . . . . . 78

38 Prozentuale Abweichung der einzelnen Varianten . . . . . . . . . . 90

39 Wandtemp.: Variante 'ohne Laibung / ohne Heizung' $(10 \mathrm{~cm}) . \quad$. . . 91

40 Wandtemp.: Variante 'mit Laibung / ohne Heizung' $(10 \mathrm{~cm})$. . . . . 92

41 Wandtemp.: Variante 'mit Laibung / mit Heizung' $(10 \mathrm{~cm})$. . . . . . 93

42 Oberflächentemperaturen am Boden und der Decke (10cm). . . . . 94

43 Lufttemp.: Variante 'mit Laibung / ohne Heizung' $(6 \mathrm{~cm})$. . . . . . 95

44 Wandtemp.: Variante 'mit Laibung / ohne Heizung' $(6 \mathrm{~cm})$. . . . . 96

45 Lufttemp.: Variante 'mit Laibung / mit Heizung' $(6 \mathrm{~cm})$. . . . . . . 97

46 Wandtemp.: Variante 'mit Laibung / mit Heizung' $(6 \mathrm{~cm})$. . . . . . 98

47 Zeitlicher Verlauf der Lufttemperaturen A/B $(6 \mathrm{~cm}) \ldots . . . . . .99$

48 Zeitlicher Verlauf der Boden- und Deckentemperaturen $(6 \mathrm{~cm}) \quad$. . . 100

49 Lufttemp.: Variante 'ohne Laibung / ohne Heizung' $(3 \mathrm{~cm})$. . . . . 102

50 Wandtemp.: Variante 'ohne Laibung / ohne Heizung' $(3 \mathrm{~cm})$. . . . 103

51 Lufttemp.: Variante 'mit Laibung / ohne Heizung' $(3 \mathrm{~cm})$. . . . . . 104

52 Wandtemp.: Variante 'mit Laibung / ohne Heizung' $(3 \mathrm{~cm})$. . . . . 105

53 Lufttemp.: 'mit Laibung / mit Heizung' $(3 \mathrm{~cm})$. . . . . . . . . 106

54 Lufttemp.: 'mit Laibung / mit Heizung' $(3 \mathrm{~cm})$. . . . . . . . . . . 107

55 Zeitlicher Verlauf der Lufttemperaturen A/B $(3 \mathrm{~cm}) \ldots . . . . . .108$

56 Zeitlicher Verlauf der Boden- und Deckentemperaturen $(3 \mathrm{~cm})$. . . 109 


\section{Tabellenverzeichnis}

1 Randbedingungen der drei Literaturansätze . . . . . . . . . . . . 18

2 Vergleich der gemessenen und berechneten red. Höhe h . . . . . . 72

3 Vergleich der gemessenen und berechneten Faktoren Z . . . . . . . 72

4 Discharge-Koeffizienten und Strömungsexponenten . . . . . . . . 75

5 Grenzen für den thermisch induzierten Luftwechsel . . . . . . . . . 81 